Linköping University Medical Dissertation

No. 1448

\title{
Immune regulation at the fetal-maternal interface with focus on decidual macrophages
}

\section{Judit Svensson-Arvelund}

\section{Linköping University}

Clinical Immunology and Obstetrics and Gynecology,

Department of Clinical and Experimental Medicine,

Faculty of Health Sciences, Linköping University,

SE-581 85 Linköping

Linköping 2015 
Copyright (C) Judit Svensson-Arvelund, 2015

Re-use allowed under a Creative Commons by attribution license (CC-BY)

http://creativecommons.org/licenses/by/4.0/

Cover image: Photography of "Studies of Embryos" (Leonardo da Vinci ) by Luc

Viatour, www.Lucnix.be

Published papers have been reprinted with permission from the copyright holders:

Paper I. Copyright 2011. The American Association of Immunologists, Inc.

Paper III. Copyright 2015. The American Association of Immunologists, Inc.

ISBN: 978-91-7519-117-1

ISSN: 0345-0082

Printed by LiU-tryck, Linköping, Sweden, 2015 
"Research is formalized curiosity. It is poking and prying with a purpose", -Zora Neale Hurston 


\section{Supervisor}

Jan Ernerudh, Linköping University, Sweden

\section{Co-supervisors}

Maria Jenmalm, Linköping University, Sweden

Göran Berg, Linköping University, Sweden

\section{Faculty opponent}

Siamon Gordon, University of Oxford, UK

\section{Funding}

This work was supported by the Swedish Research Council, the County Council of Östergötland and Linköping University, the Research Council of Southeast Sweden (FORSS) and the LIONS Medical Research Foundation. 


\section{Abstract}

A successful pregnancy requires that the maternal immune system adapts to tolerate the semi-allogeneic fetal-placental unit. This adaptation mainly occurs locally, i.e. at the fetal-maternal interface, where fetal-derived tissues come into close contact with maternal cells in the uterine endometrium (called decidua during pregnancy). Macrophages and regulatory $\mathrm{T}$ (Treg) cells are maternal immune cells that are enriched in the decidua and they likely play a central role in promoting fetal tolerance. However, the precise function of decidual macrophages and the factors regulating both macrophages and Treg cells in humans are unknown. The aim of this thesis was to characterize the phenotype and function of decidual macrophages from first trimester human pregnancy and to identify factors responsible for inducing tolerogenic properties in both decidual macrophages and Treg cells. $\mathrm{CD}_{1}{ }^{+}$decidual macrophages showed characteristics of immune suppressive or homeostatic macrophages (expression of CD163, CD206 and CD209), mainly produced immunosuppressive cytokines, like IL-10 and IL-35, while levels of inflammatory cytokines, for instance IL-12 and IL-23, were low. Decidual macrophages also induced the expansion of CD2 $5^{\text {high }} \mathrm{Foxp}^{+}$Treg cells, but not of Th1, Th2 and Th17 cells, in vitro. In addition, decidual macrophages preferentially secreted the monocyte- and Treg cell-associated chemokines CCL2 and CCL18, while Th1-, Th2- and Th17-related chemokines were produced at low levels. These results suggest that decidual macrophages contribute to create the unique decidual cell composition and a tolerogenic immune environment that is compatible with fetal development. Further, by comparing decidual macrophages with different in vitro macrophage subsets, we showed that M-CSF and IL-10, but not GM-CSF, Th1 or Th2 stimuli, induced macrophages that resemble decidual macrophages in terms of cell surface marker expression, cytokine and chemokine production and gene expression profile. First trimester placental tissue, in particular placental trophoblast cells, was identified as an important source of M-CSF and IL-10. We also demonstrated that human fetal-derived placental tissue can induce the characteristics of decidual macrophages $\left(\mathrm{CD} 163^{+} \mathrm{CD} 206^{+} \mathrm{CD} 209^{+} \mathrm{IL}-10^{+} \mathrm{CCL} 18^{+}\right)$ and the selective expansion of functionally suppressive $\mathrm{CD} 25^{\text {high }}$ Foxp $3^{+}$Treg cells, the 
latter partly mediated through IL-10, TGF- $\beta$ and TRAIL. The placenta also limited activation of Th cells, for instance by generally reduced cytokine production. Our data show that the placenta has a unique ability to induce tolerogenic immune cells with a reduced inflammatory potential, which is essential for maintaining tissue integrity and preventing inflammation-induced fetal loss. 


\section{Table of Contents}

Sammanfattning...................................................................................................................

Original publications....................................................................................................

Supplemental relevant publications...............................................................................9

Abbreviations ........................................................................................................11

Introduction ........................................................................................................................13

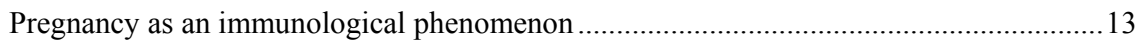

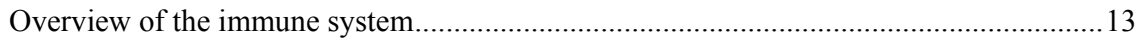

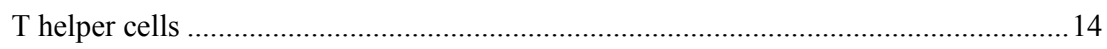

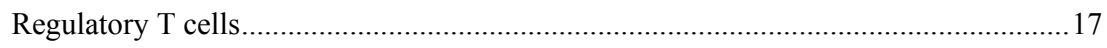

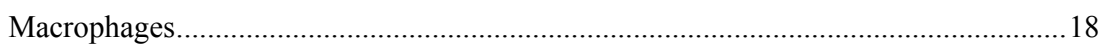

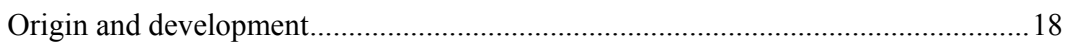

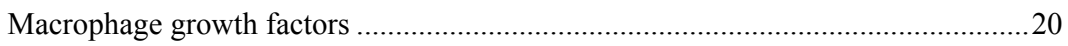

Effect of growth factors on macrophage function ...................................................2 21

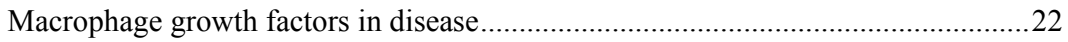

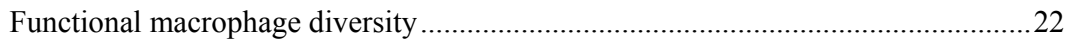

Macrophage activation and polarization .............................................................2

Extended nomenclature and classification of macrophage polarization....................25

Markers differentially expressed on macrophage subtypes ....................................27

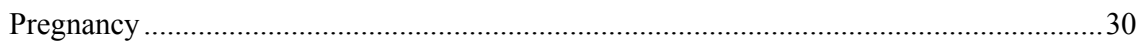

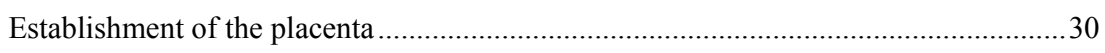

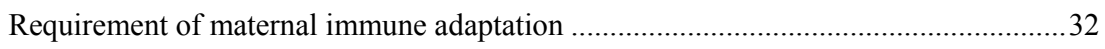

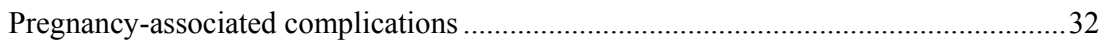

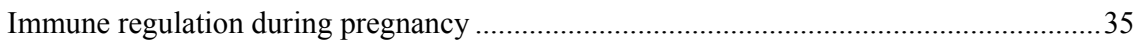

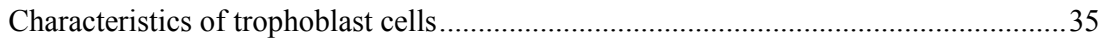

Systemic adaptations of the maternal immune system ..................................................36

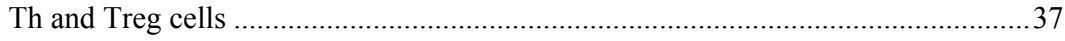

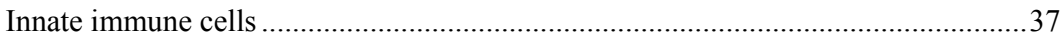

Maternal immune adaptation at the fetal-maternal interface.........................................39 


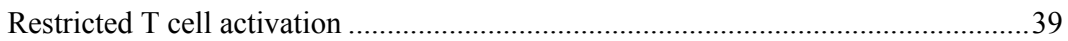

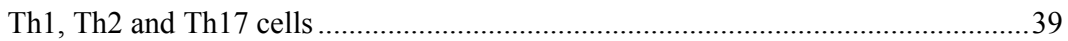

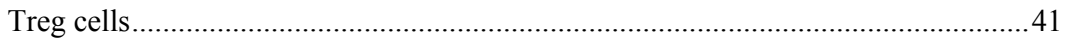

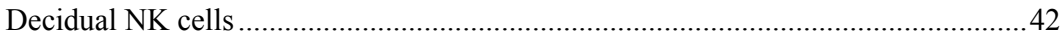

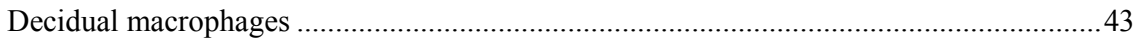

Phenotype and function of decidual macrophages ........................................................4

Origin and development of decidual macrophages .......................................................46

The role of decidual macrophages in pregnancy complications.....................................4

Aims and hypotheses .........................................................................................................49

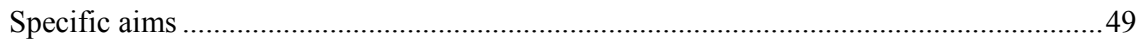

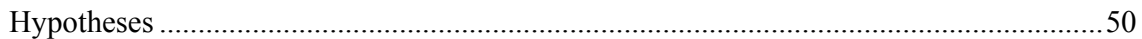

Study design and methodological considerations .............................................51

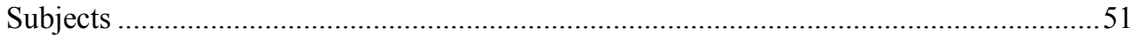

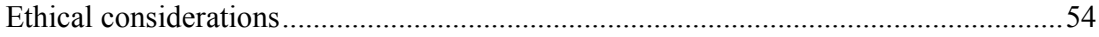

First trimester samples as a model of healthy pregnancy ..............................................54

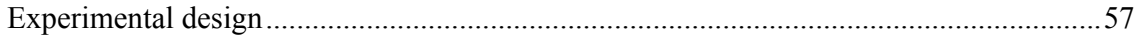

In vitro polarization of macrophages.........................................................................5

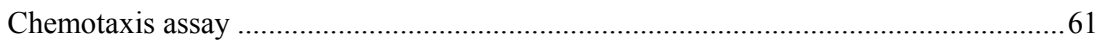

Th cell polarization; unstimulated versus stimulated cells ...........................................63

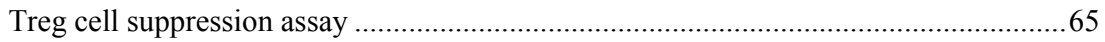

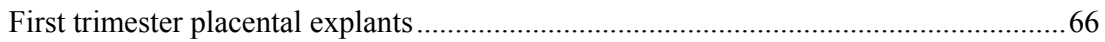

First trimester primary trophoblast cells and the HTR-8/SVneo trophoblast cell line...68

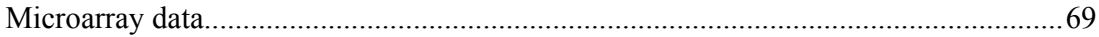

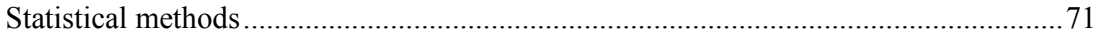

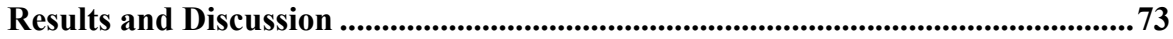

Phenotype and subpopulations of decidual macrophages ................................................... 73

The influence of M-CSF and IL-10 on decidual macrophage polarization .........................76

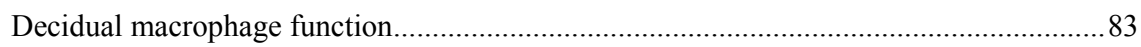

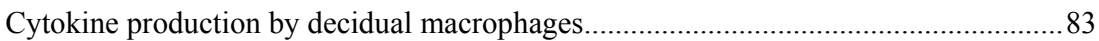

Influence of decidual macrophages on the polarization of Th1, Th2, Th17 and Treg

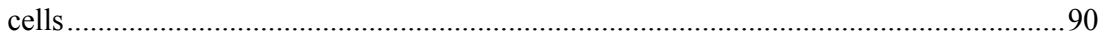

A potential role for decidual macrophages in controlling cell trafficking at the fetal-

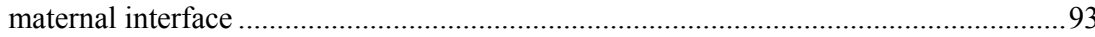




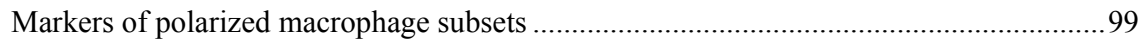

Role of the placenta in promoting a homeostatic immune environment........................... 101

Induction of homeostatic M2 macrophages and Treg cells ........................................ 101

The HTR-8/SVneo cell line differs from primary trophoblast cells..............................108

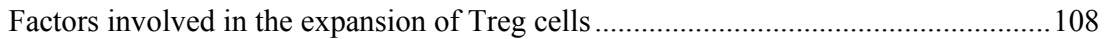

Summary and conclusions ....................................................................................................111

Future perspectives.......................................................................................................115

Acknowledgements ..................................................................................................119

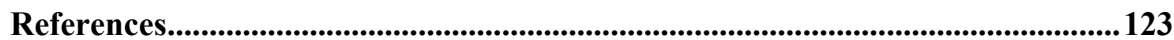




\section{Sammanfattning}

Ur ett immunologiskt perspektiv är graviditet ett utmanande tillstånd, då fostret till hälften utvecklas av gener från pappan och därför är delvis främmande för mammans immunförsvar. Detta innebär att immunförsvaret behöver anpassas för att förhindra avstötning av fostret, samtidigt som det måste behålla sin förmåga att skydda kroppen mot infektioner. Det lokala immunförsvaret hos mamman består av de immunceller som finns i livmodern (kallad decidua under graviditeten). Dessa har en central roll i anpassningen på grund av den nära kontakten både med fostret och med moderkakan (placentan), som utvecklas från samma celler som fostret. Vi har studerat immunceller i decidua som kan vara viktiga i anpassningen till det främmande fostret. Makrofager är immunceller med förmåga att reglera omgivningen; hur dessa utövar sin immundämpande förmåga under graviditet är dock oklart. En annan celltyp som visats ha betydelse för graviditet är regulatoriska T-celler som är viktiga för att skapa tolerans och förhindra överdriven inflammation. De specifika faktorer som styr makrofagernas och de regulatoriska T-cellernas immundämpande egenskaper är dock inte klarlagda.

Det övergripande syftet med de delarbeten som ingår i avhandlingen var att kartlägga decidua-makrofagernas egenskaper samt att identifiera de faktorer som styr utvecklingen av både makrofager och regulatoriska T-celler i deciduan. Eftersom placentan är det nya och främmande organet vid graviditet, studerades om placentaproducerade faktorer kunde skapa de unika egenskaperna som makrofager och Tceller antar vid en graviditet. Decidua-makrofager och placenta från första trimester graviditet undersöktes, liksom deras effekt på immunceller i blodprover från ickegravida kvinnor.

Studierna visade att decidua-makrofager huvudsakligen producerar signalsubstanser (cytokiner) med immunhämmande egenskaper, som IL-10 och IL-35, och kan öka antalet regulatoriska T-celler men inte antalet konventionella T-celler (Th1, Th2 och Th17) som aktiverar immunförsvaret. Decidua-makrofager producerade också höga nivåer av faktorer (kemokiner) som reglerar celltrafik, framförallt producerades 
kemokiner som rekryterar omogna makrofager och regulatoriska T-celler, vilket visar att makrofagerna har en viktig roll i att skapa den unika miljö som är nödvändig för utvecklingen av en normal graviditet. Genom att testa en rad olika faktorer experimentellt, identifierades tillväxtfaktorn M-CSF och det immundämpande cytokinet IL-10 som viktiga ämnen i makrofagernas reglering.

I det sista delarbetet visade vi att faktorer som placentan spontant producerar kan styra utvecklingen av både regulatoriska T-celler och immunreglerande makrofager.

Placenta-faktorer kunde även hindra en generell immunaktivering, bland annat genom att hämma utvecklingen av aggressiva T-celler. Genom att blockera specifika substanser, identifierades M-CSF och IL-10 som viktiga för utvecklingen av de immunhämmande makrofagerna, vilket visar att placentan är en viktig källa till MCSF och IL-10. IL-10 identifierades även som en av flera faktorer viktiga för regulatoriska T-celler. Placentan, som utgör det främmande organet under graviditet, har alltså en unik och inbyggd förmåga att skapa immunologisk tolerans och därmed säkra fostrets utveckling. Eftersom substanser som IL-10 och M-CSF är viktiga för normal graviditet kan avvikelser i dessa vara inblandade $\mathrm{i}$ onormal graviditet och framtida behandlingar mot graviditetskomplikationer kan komma att inriktas på att återställa dessa substanser. 


\section{Original publications}

I. Macrophages at the fetal-maternal interface express markers of alternative activation and are induced by M-CSF and IL-10.

Judit Svensson*, Maria C. Jenmalm, Andreas Matussek, Robert Geffers, Göran Berg, Jan Ernerudh

J Immunol, 2011, 187: 3671-3682

II. Decidual macrophages contribute to the unique leukocyte composition at the fetal-maternal interface by production of IL-35, induction of Treg cells and production of homeostatic chemokines.

Judit Svensson-Arvelund, Daniel Söderberg, Caroline Wendel, Sofia Freland, Robert Geffers, Göran Berg, Maria C. Jenmalm, Jan Ernerudh

Manuscript

III. The human fetal placenta promotes tolerance against the semiallogeneic fetus by inducing regulatory $\mathrm{T}$ cells and homeostatic $\mathrm{M} 2$ macrophages.

Judit Svensson-Arvelund, Ratnesh B. Mehta, Robert Lindau, Elahe Mirrasekhian, Heriberto Rodriguez-Martinez, Göran Berg, Gendie E. Lash, Maria C. Jenmalm, Jan Ernerudh

J Immunol, 2015, 194: 1534-1544

*The author's maiden name is Svensson 


\section{Supplemental relevant publications}

SI. Systemic reduction of functionally suppressive $\mathrm{CD}^{\mathrm{dim}} \mathrm{CD}^{\mathrm{d}} 5^{\text {high }} \mathrm{Foxp3}^{+}$Tregs in human second trimester pregnancy is induced by progesterone and $17 \beta$ estradiol.

Jenny Mjösberg, Judit Svensson*, Emma Johansson, Lotta Hellström, Rosaura Casas, Maria C. Jenmalm, Roland Boij, Leif Matthiesen, Jan-Ingvar Jönsson, Göran Berg, Jan Ernerudh.

J Immunol, 2009, 183: 759-769.

SII. Biomarkers of coagulation, inflammation, and angiogenesis are independently associated with preeclampsia.

Roland Boij, Judit Svensson*, Kristina Nilsson-Ekdahl, Kerstin Sandholm, Tomas L. Lindahl, Elzbieta Palonek, Mats Garle, Göran Berg, Jan Ernerudh, Maria C. Jenmalm, Leif Matthiesen.

Am J Reprod Immunol, 2012, 68:258-270.

SIII. The placenta in toxicology. Part II: Systemic and local immune adaptations in pregnancy.

Judit Svensson-Arvelund, Jan Ernerudh, Eberhard Buse, J. Mark Cline, Jan-Dirk Haeger, Darlene Dixon, Udo R. Markert, Christiane Pfarrer, Paul De Vos, Marijke M. Faas.

Toxicol Pathol, 2014, 42:327-338.

SIV. The role of macrophages in promoting and maintaining homeostasis at the fetal-maternal interface.

Judit Svensson-Arvelund, Jan Ernerudh.

Am J Reprod Immunol, 2015. doi: 10.1111/aji.12357. [Epub ahead of print]

*The author's maiden name is Svensson 


\section{Abbreviations}

APC Antigen-presenting cell

CFSE Carboxyfluorescein diacetate succinimidyl ester

CM Conditioned medium

CTB cells Cytotrophoblast cells

CTLA Cytolytic T lymphocyte-associated antigen

DC Dendritic cell

DC-SIGN Dendritic cell-specific intercellular adhesion molecule-3-grabbing nonintegrin

EGF Epidermal growth factor

EVT cells Extravillous trophoblast cells

FACS Flow cytometry-activated cell sorting

FasL Fas-ligand

FDR False discovery rate

GM-CSF Granulocyte-macrophage colony-stimulating factor

hCG Human chorionic gonadotrophin

HTR8 HTR-8/SVneo

ICAM Intercellular adhesion molecule

IDO Indoleamine 2,3-dioxynenase

IHC Immunohistochemistry

IL Interleukin

ILC2 Group 2 innate lymphoid cells

IFN Interferon

IRF Interferon regulatory factor

LIF Leukaemia inhibitory factor

LPS Lipopolysaccharide

MACS Magnetic-activated cell sorting

M-CSF Macrophage colony-stimulating factor

MFI Mean fluorescence intensity

MHC Major histocompatibility complex 
MR Mannose receptor

NK cell Natural killer cell

NRP-1 Neuropilin-1

PBMC Peripheral blood mononuclear cells

PGE1 Prostaglandin E1

PE CM Placental explant CM

PRR Pattern recognition receptor

SR Scavenger receptor

TAM Tumor-associated macrophage

TCR T cell receptor

TGF- $\beta \quad$ Transforming growth factor- $\beta$

Th cell Thelper cell

TLR Toll-like receptor

TNF Tumor necrosis factor

TRAIL TNF-related apoptosis-inducing ligand

Treg cell Regulatory $\mathrm{T}$ cell

TSLP Thymic stromal lymphopoietin

VEGF Vascular endothelial growth factor

VSMC Vascular smooth muscle cells 


\section{Introduction}

\section{Pregnancy as an immunological phenomenon}

The ability of the immune system to distinguish between self and non-self and to mount an immune response to non-self or foreign antigens is central for the protection against potentially harmful pathogens. Therefore, the semi-allogeneic nature of the fetus implicates a great challenge during pregnancy, because the maternal immune system needs to adjust to tolerate the fetus while maintaining protective immunity against infections. In this thesis, I will discuss the maternal immune adaptations that occur at the fetal-maternal interface in healthy human pregnancy. The focus will be on the role of decidual macrophages in promoting immune homeostasis, but the role of the placenta in promoting an immune microenvironment that is compatible with normal fetal development will also be considered.

\section{Overview of the immune system}

The immune system is traditionally divided into innate and adaptive immunity (Abbas et al., 2015). The innate immune system represents the first line of defense and responds rapidly to common components of microorganisms. The adaptive immune system requires longer time to develop but is more specific and can develop memory to encountered antigens. Although these systems are generally described separately, they are closely linked and an efficient immune response is dependent on the interaction between the cellular and molecular components of the innate and the adaptive immune system. Macrophages are a central component of innate immunity, and an important aspect of macrophage function is the ability to influence adaptive immune responses, in particular the polarization of $\mathrm{CD}^{+} \mathrm{T}$ helper and regulatory $\mathrm{T}$ cells, which will be introduced in this chapter. 


\section{T helper cells}

$\mathrm{CD}^{+} \mathrm{T}$ helper (Th) cells have a central role in adaptive immunity by orchestrating immune responses to pathogens (Abbas et al., 2015). Th cells exit the thymus as naïve Th cells and recirculate between the blood and secondary lymphoid organs. Activation occurs within secondary lymphoid organs by the recognition of antigens associated with antigen-presenting cells (APCs). Dendritic cells (DCs) are the major APCs during the initial activation of naïve Th cells, but macrophages and B cells can also present antigens to Th cells. Th cells recognize antigens through their $\mathrm{T}$ cell receptor (TCR)-CD3 complex, which binds the antigen-major histocompatibility complex class II (MHC II) complex on APCs. Efficient activation is dependent on the interaction between co-stimulatory molecules on APCs, such as CD80, CD86 and CD40, and their receptors on T cells, such as CD28 and CD40 ligand. The presence of different cytokines during the activation process drives the differentiation into distinct Th cell subsets. A summary of key aspects of Th cells is shown in figure 1 .

Th cells may differentiate into three major effector subsets, Th1, Th2 or Th17 cells (Annunziato and Romagnani, 2009; Zhu and Paul, 2010). Th9 and Th22 cell subsets have also been described, although their role in protective immunity is not clear. Naïve Th cells can also differentiate into inducible regulatory T (Treg) cells, which are central for the regulation of effector cells during inflammation (described below).

Th1 cells are critical for the protection against intracellular pathogens, for instance against mycobacterial infections, but they can also contribute to tissue damage during chronic inflammation and autoimmune diseases. The major Th1-inducing cytokines are interleukin (IL)-12, mainly produced by macrophages and DCs, and interferon (IFN)- $\gamma$ produced by natural killer (NK) cells, in response to microbes (Hsieh et al., 1993; Mosser and Edwards, 2008). These cytokines induce the activation of several transcription factors, including STAT1, STAT4 and T-bet (Zhu and Paul, 2010). T-bet is the master regulator of Th1 cells and promotes production of IFN- $\gamma$, which in turn serves to amplify the Th1 response (Szabo et al., 2000). IFN- $\gamma$ promotes the activation of classically activated macrophages (described in more detail later on) enhancing 
Th1

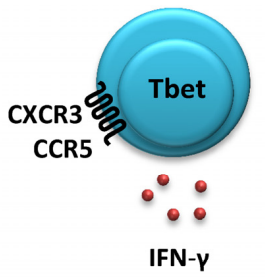

IFN- $\boldsymbol{V}$

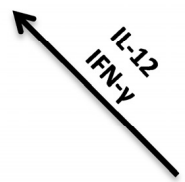

Th17

IL-17

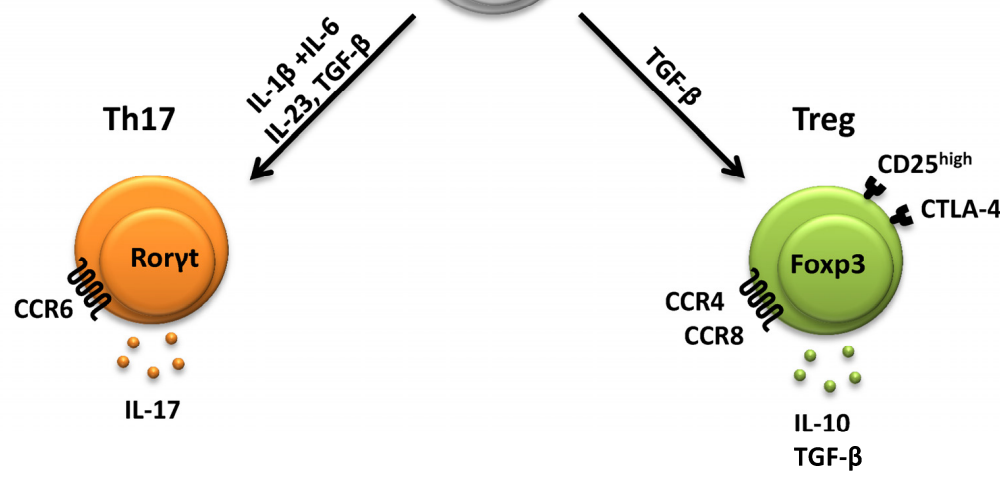

Th2

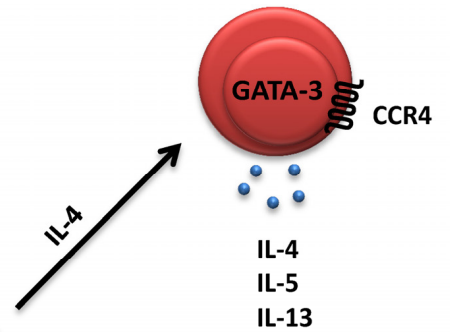

IL-13

Figure 1. Schematic representation of $\mathrm{CD}^{+} \mathrm{T}$ helper cell differentiation.

microbicidal activity that is necessary to destroy intracellular pathogens (Mosser and Edwards, 2008). IFN- $\gamma$ also provides macrophages with enhanced antigen-presenting capacity, for instance by increased MHC expression, which further promotes Th1 cell activation.

Th2 cells are central for the host protection against extracellular parasites and they are also involved in the development of allergic diseases (Islam and Luster, 2012). IL-4 drives the differentiation of Th2 cells through the activation of the transcription factors STAT6 and GATA-3. GATA-3 is the master regulator of Th2 differentiation and induces expression of IL-4, IL-5 and IL-13 (Zheng and Flavell, 1997; Zhu and Paul, 2008). These cytokines promote IgE antibody responses and the activation of 
mast cells and eosinophils that are involved in the defense against extracellular parasites. Th2 cytokines also induce alternatively activated macrophages with tissue remodeling properties that differ from the pro-inflammatory macrophages induced by IFN- $\gamma$ (described in more detail in later sections).

Th17 cells are responsible for the immune defense against extracellular bacteria and fungi and are also involved in the development of organ-specific autoimmune diseases (Annunziato et al., 2012). The differentiation of Th17 cells is induced by IL-6 and IL$1 \beta$, while IL-23 is necessary for the maturation and pathogenicity of Th17 cells (Annunziato et al., 2012; Gaffen et al., 2014). In addition, although some in vitro studies have shown that Th17 cells may differentiate in the absence of transforming growth factor (TGF)- $\beta$, several studies have demonstrated its requirement and the presence of TGF- $\beta$ is believed to be necessary for optimal Th17 differentiation in vivo (Gaffen et al., 2014). Th17 differentiation involves activation of the transcription factor STAT3 and the master regulator Roryt. Roryt induces production of IL-17 (IL17A and IL-17F) that mediates most of the effects of Th17 cells (Ivanov et al., 2006; Annunziato et al., 2012). For instance, IL-17 promotes upregulation of the chemokine CXCL8 from several cell types, including epithelial cells and macrophages, thus leading to the recruitment of neutrophils to the site of infection (Annunziato et al., 2012). Th17 cells also produce tumor necrosis factor (TNF) and granulocytemacrophage colony-stimulating factor (GM-CSF) that promote survival and activate neutrophils, and IL-21 that serves to amplify the immune response by promoting Th17 differentiation.

Activated Th cells migrate to sites of infection where they perform their effector functions and the specific recruitment is largely mediated by chemokines produced at sites of infection (Griffith et al., 2014). Th1 cells preferentially express the chemokine receptor CXCR3, which binds to CXCL9, CXCL10 and CXCL11, typically induced by IFN- $\gamma$, and CCR5 that binds to CCL5 produced during inflammation (Qin et al., 1998; Zhu and Paul, 2008). Th2 cells are mainly characterized by expression of CCR4 that binds to CCL17 and CCL22 (Islam and Luster, 2012) but have also been shown to migrate in response to CCL1 and CCL18 through CCR8 (Islam et al., 2013). Th17 
cells are mainly recruited by CCL20, induced by IL-17, upon binding to its receptor CCR6 (Annunziato et al., 2012).

An important feature of Th cells is the cross-regulation across subsets that, in addition to amplification through positive feedback loops, ensure that the most appropriate type of immune response if efficiently induced. For example, Th1 and Th2 cytokines and transcription factors suppress each other (Ferber et al., 1999; Szabo et al., 2000) and both IFN- $\gamma$ and IL-4 inhibit development of Th17 cells (Harrington et al., 2005; Park et al., 2005).

\section{Regulatory T cells}

In addition to conventional Th cells, $\mathrm{CD} 4^{+} \mathrm{T}$ cells can also differentiate into Treg cells that are essential for the regulation of inflammatory responses to pathogens but also for peripheral tolerance and the protection against autoimmune diseases. There are two main types of Treg cells, thymic (also called natural) Treg cells that are generated in the thymus and are believed to protect against self-reactive immune responses, and peripheral (also called inducible) Treg cells that are generated in peripheral tissues and may have specificity to self and foreign antigens (Workman et al., 2009). Among peripheral Treg cells, in addition to Foxp $3^{+}$Treg cells (described below), two major types have previously been described; IL-10-producing Tr1 cells induced upon stimulation with IL-10 (Groux et al., 1996), and Th3 cells generated by TGF- $\beta$ (Chen et al., 2003). Most recently, the anti-inflammatory cytokine IL-35 (Collison et al., 2007) was also shown to induce T cells that suppressed through the same cytokine, IL-35, but independently of IL-10 and TGF- $\beta$ (Collison et al., 2010).

The major group of Treg cells is however defined by expression of the transcription factor Foxp3 (Miyara and Sakaguchi, 2011), and includes both thymic Treg cells and certain inducible subsets, for instance those induced by TGF- $\beta$ (Workman et al., 2009). These Treg cells were originally described in mice as $\mathrm{CD}^{+} \mathrm{CD} 25^{+}$(Sakaguchi et al., 1995) and were later shown to be regulated by the transcription factor Foxp3 (Fontenot et al., 2003). In humans, CD25 and Foxp3 are upregulated upon activation 
in non-suppressive Th cells (Baecher-Allan et al., 2001; Wang et al., 2007), thus the phenotype of human Treg cells has not been straightforward. However, high expression of CD25 (the IL-2 receptor $\alpha$-chain) and stable expression of Foxp3 is associated with suppressive activity in human Treg cells. In addition, expression of CD127 (the IL-7 receptor $\alpha$-chain), was found to inversely correlate with the expression of Foxp3 and accordingly, Treg cells may also be defined as CD127 $7^{\text {low }}$ (Liu et al., 2006). Other markers shown to be expressed by human Treg cells are cytolytic T lymphocyte-associated antigen (CTLA)-4 that competes with CD28 and thus inhibits co-stimulation through CD80/CD86 on APCs, and CD39, an ectonucleotidase that mediates immune suppression by inactivating ATP (Borsellino et al., 2007; Wing et al., 2008; Miyara and Sakaguchi, 2011). Treg cells mediate suppression through cell-contact, for instance by CTLA-4, or by the production of anti-inflammatory cytokines, such as IL-10 and TGF- $\beta$ (Wing et al., 2008; Workman et al., 2009).

Similar to Th2 cells, the recruitment of Treg cells from blood to peripheral tissues can be mediated by the chemokine receptor CCR4 in response to CCL17 and CCL22 (Iellem et al., 2001; Griffith et al., 2014). In addition, CCR8 has been shown to preferentially attract Treg cells in response to CCL1 and CCL18 (Iellem et al., 2001; Bellinghausen et al., 2012; Chenivesse et al., 2012; Islam et al., 2013). Recent data also suggest that Treg cells are heterogeneous and may be divided into distinct subtypes with features, including chemokine receptor expression, associated with Th1, Th2 or Th17 cells (Duhen et al., 2012; Tian et al., 2012). Thus, Treg cells may be activated in parallel with Th cell subsets and could co-migrate with effector Th cells to sites of infection ensuring the effective control of immune responses.

\section{Macrophages}

\section{Origin and development}

Macrophages reside within almost every tissue and display different phenotypes and functions depending on the tissue-specific requirements (Murray and Wynn, 2011; Davies et al., 2013; Wynn et al., 2013; Gordon et al., 2014). The traditional view has been that adult tissue macrophages originate from myeloid cell precursors in the bone 
marrow. In this model, myeloid precursors mature into monocytes, enter the circulation and migrate to the tissues where they mature into macrophages both during homeostasis and inflammation (Geissmann et al., 2010). However, recent data based on lineage-tracing mouse models have revealed that several tissue macrophage populations (for instance in the skin, brain, lung and liver) originate from myeloid progenitors in the yolk sac or fetal liver during embryonic development and persist until adulthood (Ginhoux et al., 2010; Hoeffel et al., 2012; Schulz et al., 2012). Recent data also show that most tissue macrophages are not dependent on the replacement by bone marrow-derived blood monocytes, neither at the steady state nor during certain infections (like Th2-driven parasitic infections); instead they selfmaintain or accumulate by proliferating within the tissues (Jenkins et al., 2011; Hashimoto et al., 2013). In contrast, some tissues have been shown to be dependent on the continuous recruitment of blood monocytes to maintain tissue macrophage numbers under non-inflammatory conditions, for instance the gastrointestinal tract (Bain et al., 2013) and the pregnant uterus (Tagliani et al., 2011). However, it is likely that many tissues are populated by heterogeneous macrophage populations originating from embryonic myeloid precursors as well as from blood-derived monocytes (Schulz et al., 2012; Ginhoux and Jung, 2014).

In parallel with findings that blood monocytes do not significantly contribute to the replacement of macrophages in most tissues in mice, there has been increasing knowledge on monocyte biology. Two major blood monocyte subsets can be identified in humans, the classical $\mathrm{CD} 14^{++} \mathrm{CD} 16^{-}$and the non-classical $\mathrm{CD} 14^{+/ \text {low }} \mathrm{CD} 16^{+}$subsets (Tacke and Randolph, 2006; Ancuta et al., 2009; ZieglerHeitbrock et al., 2010). Classical monocytes account for $\sim 90-95 \%$ of all blood monocytes, express CCR2 (Weber et al., 2000) and are believed to be recruited to tissues during infections or to tissues that depend on the continuous recruitment of monocytes, like the gut and uterus (Shi and Pamer, 2011; Wynn et al., 2013). In contrast, the non-classical monocytes ( $\sim 5-10 \%$ in blood) lack CCR2 expression and have been described as patrolling blood-resident cells whose function is to maintain endothelial integrity (Cros et al., 2010). 


\section{Macrophage growth factors}

Regardless of their origins, macrophages are critically dependent on growth factors for their survival and development (Hamilton and Achuthan, 2013; Wynn et al., 2013). Important macrophage growth factors include GM-CSF (also known as CSF-2), MCSF (also known as CSF-1) and the most recently discovered IL-34 that shares receptor with M-CSF (Lin et al., 2008). Although originally described as hematopoietic-cell growth factors (at least M-CSF and GM-CSF) (Morstyn and Burgess, 1988), it has become increasingly recognized that these factors not only act during the early developmental stages of myeloid cells, but can also influence the function of mature macrophage populations. M-CSF is the most abundant of the macrophage growth factors, being constitutively produced by many cell types and being present in the circulation during homeostatic conditions (Hamilton, 2008). The relevance of M-CSF for the development and maintenance of tissue macrophages has largely been defined by studies on $C s f 1^{o p} / C s f 1^{o p}$ mice, which are homozygous for an inactivating mutation in the gene encoding M-CSF (Wiktor-Jedrzejczak et al., 1990). These mice suffer from widespread macrophage deficiencies, for instance in the bone marrow, kidney and uterus, while some tissues are only partially or not at all affected, for instance the brain and skin (Wiktor-Jedrzejczak and Gordon, 1996). IL-34, that shares receptor with M-CSF, shows similar effects on macrophage differentiation but appears to be restricted to certain macrophage populations; to date, IL-34 has mainly been shown to regulate the development of microglia and Langerhans cells in mice (Greter et al., 2012b; Wang et al., 2012). In contrast to the constitutive production of M-CSF, GM-CSF is found at low levels in the circulation at the steady state and its detection often requires cell stimulation (Hamilton, 2008). GM-CSF-deficient mice show normal macrophage development in most tissues, with the exception of lung macrophages that show severe deficiencies leading to pulmonary disease (Stanley et al., 1994). Instead, GM-CSF appears to be involved in the homeostatic maintenance of DCs in non-lymphoid tissues (Greter et al., 2012a). 


\section{Effect of growth factors on macrophage function}

In addition to spatial differences, macrophage growth factors have different effects on the function of mature macrophages. Most knowledge about the effects of M-CSF and GM-CSF (and to a lesser extent also IL-34) on macrophage differentiation and function is derived from studies where macrophages have been generated in vitro from blood monocytes (particularly in the case of human macrophages). By this approach, M-CSF has been demonstrated to induce macrophages with predominantly antiinflammatory properties. These include high production of IL-10 and low production of pro-inflammatory cytokines such as TNF, IL-12 and IL-23, low expression of the co-stimulatory molecules CD80 and CD86 and consequently, poor capacity to induce Th1 responses (Verreck et al., 2004; Akagawa et al., 2006; Xu et al., 2006; Fleetwood et al., 2007). In addition, macrophages differentiated with M-CSF have been shown to inhibit the proliferation of $\mathrm{T}$ cells and to induce CD $25^{+} \mathrm{Foxp} 3^{+}$Treg cells (Munn et al., 1999; Savage et al., 2008). By contrast, GM-CSF promotes macrophages with low IL10 and high TNF, IL-12 and IL-23 production and high expression of CD80 and CD86 (Verreck et al., 2004; Akagawa et al., 2006; Xu et al., 2006; Fleetwood et al., 2007). Accordingly, GM-CSF macrophages promote Th1 responses and lack the ability to induce Treg cells. Similar to M-CSF, IL-34 promotes macrophages with an IL- $10^{\text {high }}$ and IL-12 ${ }^{\text {low }}$ phenotype and with low $\mathrm{T}$ cell stimulatory properties (Barve et al., 2013; Foucher et al., 2013). Importantly, these phenotypic and functional differences are largely retained after microbial challenge (Akagawa et al., 2006; Verreck et al., 2006; Foucher et al., 2013).

Macrophages generated in the presence of M-CSF and GM-CSF also differ in regards to the chemokines they produce. M-CSF mainly promotes production of the monocyte-recruiting CCL2, while neither Th1- nor Th2-associated chemokines (for instance CXCL10 and CCL22) are induced (Verreck et al., 2006; Fleetwood et al., 2007). In contrast, macrophages generated by GM-CSF do not produce CCL2, but instead express or produce CCL17 and CCL22 (Verreck et al., 2006; Lacey et al., 2012). Less is known about the chemokine repertoire of IL-34-induced macrophages; 
however, transcriptional profiling suggests that IL-34 and M-CSF macrophages have similar chemokine and chemokine receptor repertoires (Foucher et al., 2013).

\section{Macrophage growth factors in disease}

Impaired regulation of macrophage growth factors has been implicated in the development of several diseases. GM-CSF has been shown to be associated with several inflammatory and autoimmune disease models (such as arthritis, atherosclerosis and multiple sclerosis) and to be involved in both Th1 and Th17 responses (Hamilton and Achuthan, 2013). Recent data also show that GM-CSF can initiate autoimmune inflammation independently of Th1 and Th17 responses (Codarri et al., 2011; Noster et al., 2014) and that it can also drive the development of Th2associated allergic inflammation (Llop-Guevara et al., 2014). Increased M-CSF levels have also been linked to inflammatory conditions; however, the role of M-CSF in promoting inflammation is not clear and may be context-dependent (Hamilton, 2008). In contrast, much data suggests that increased M-CSF levels promote tumor growth, likely by the induction of tumor-associated macrophages (TAMs) that may suppress anti-tumor immunity (Pollard, 2004; Hamilton, 2008; Tamimi et al., 2008). Several reports have also linked elevated levels of IL-34 to inflammatory diseases (Masteller and Wong, 2014), in particular in rheumatoid arthritis where IL-34 was proposed to contribute to osteoclast formation (Hwang et al., 2012). However, overexpression of IL-34 has also been associated with tumor progression, by promoting TAMs and increasing angiogenesis (Segaliny et al., 2014).

\section{Functional macrophage diversity}

Macrophages belong to the mononuclear phagocyte system and are an important component of innate immunity. Important functions include the recognition and elimination of microorganisms, the processing and presentation of antigens to $\mathrm{T}$ cells and the production of cytokines and chemokines, which promote recruitment of leukocytes and amplification of the immune response (Mantovani et al., 2004; Benoit et al., 2008; Biswas and Mantovani, 2010; Gordon et al., 2014). Macrophages are also involved in several non-immunological processes during development and 
homeostasis, including the phagocytosis of apoptotic cells and debris, tissue remodeling, angiogenesis and regulation of metabolism (Pollard, 2009; Biswas and Mantovani, 2012; Wynn et al., 2013). Besides this functional diversity, macrophages have been described to have a high degree of plasticity, being able to adapt their phenotype and function in response to changing microenvironments (Stout and Suttles, 2004).

\section{Macrophage activation and polarization}

Based on phenotypical and functional characteristics, macrophages have commonly been categorized into distinct subsets. Originally, macrophages were described as proinflammatory cells involved in the elimination of intracellular pathogens. Classical activation is linked to Th1 responses and is typically induced by IFN- $\gamma$, initially produced by for example NK cells or at later stages by Th1 cells, and by TNF produced by macrophages themselves in response to Toll-like receptor (TLR) signals (Mosser and Edwards, 2008; Sica and Mantovani, 2012) (Fig. 2). Classically activated macrophages produce pro-inflammatory cytokines including IL-12, IL-23 and TNF, produce reactive oxygen species and express high levels of molecules associated with antigen presentation (for instance HLA-DR and CD80/CD86). Accordingly, these macrophages contribute to the elimination of intracellular microorganisms and the amplification of Th1 immune responses. In addition, if uncontrolled, classically activated macrophages may cause extensive tissue damage. When the Th2-associated cytokine IL-4 was observed to induce macrophages with reduced pro-inflammatory potential and with a phenotype distinct from the classically activated macrophages, they were termed alternatively activated (Stein et al., 1992). This phenotype is induced by IL-4 and IL-13, which are primarily produced by mast cells, basophils, group 2 innate lymphoid cells (ILC2) and Th2 cells (Martinez et al., 2009; Doherty, 2015) (Fig. 2). Alternatively activated macrophages show reduced production of proinflammatory cytokines, such as IL-12, IL-1 $\beta$ and TNF, and increased production of IL-10 and IL-1RA (IL-1 $\beta$ receptor antagonist). These cells participate in Th2 responses associated with parasitic infection, promote tissue remodeling and are 


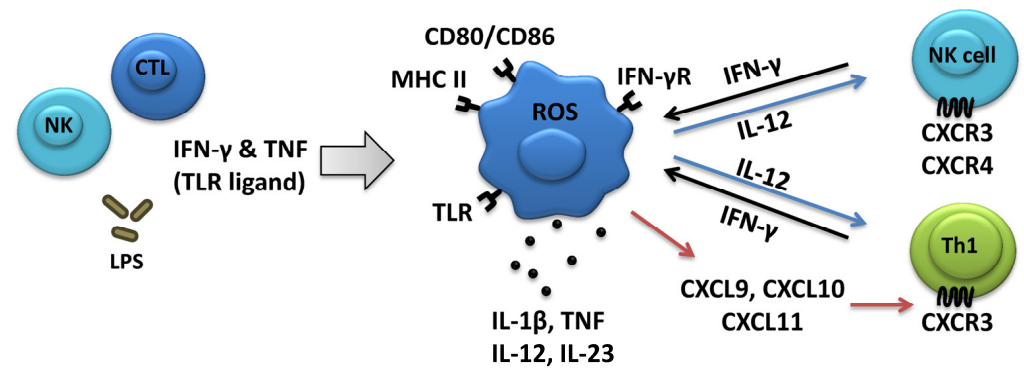

Killing of intracellular pathogens Antigen presentation Amplification of Th1

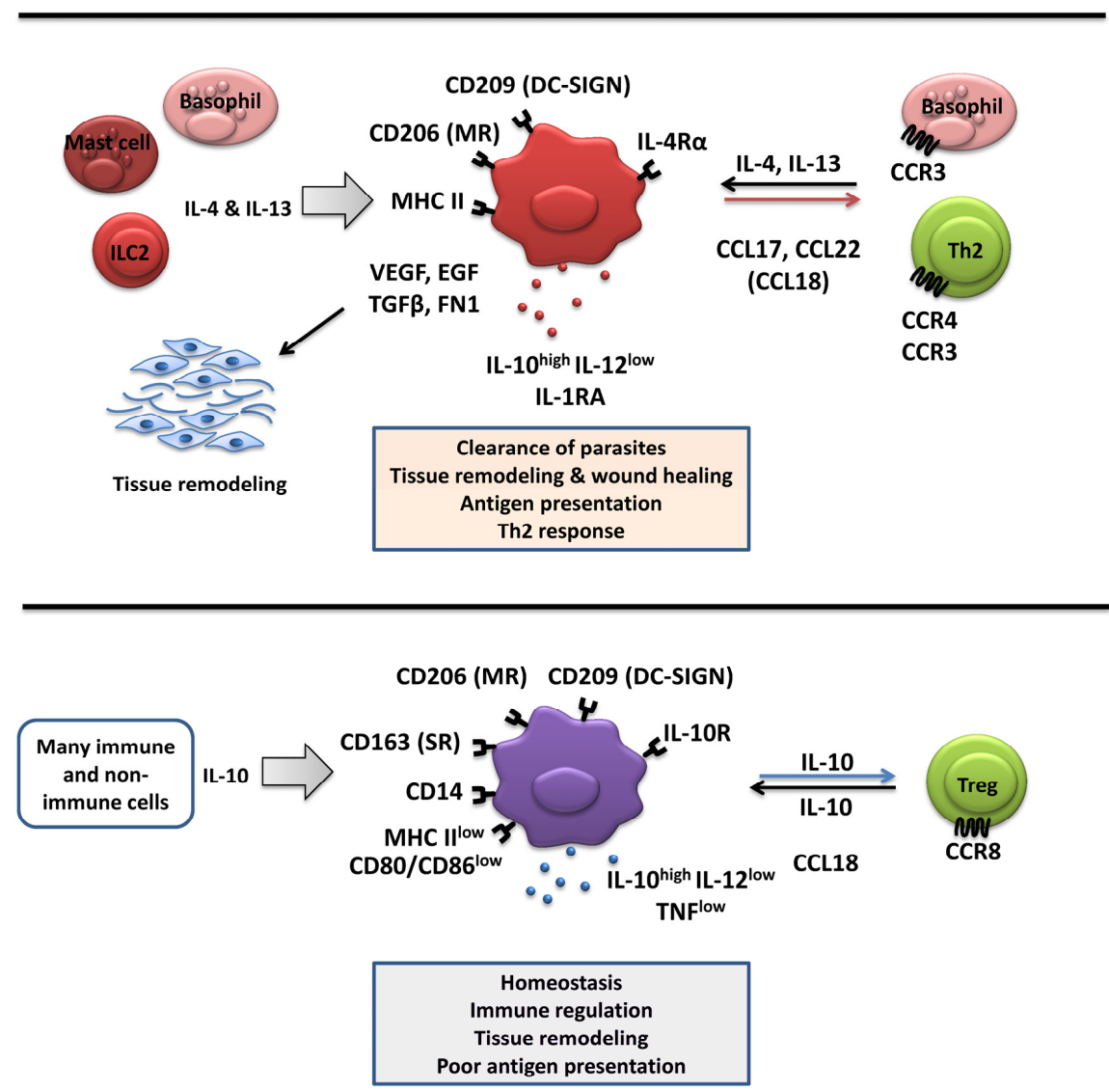


Figure 2. Schematic representation of the main characteristics of macrophages influenced by Th1 stimuli (IFN- $\gamma$ and TNF or TLR ligands), Th2 cytokines (IL-4 and IL-13) or the homeostatic cytokine IL-10. The major lymphocyte populations interacting with each macrophage subset are shown. CTL: Cytotoxic T lymphocyte, DC-SIGN: Dendritic cellspecific intercellular adhesion molecule-3-grabbing non-integrin, EGF: Epidermal growth factor, FN1: Fibronectin-1, ILC2: Group 2 innate lymphoid cell, MR: Mannose receptor, SR: Scavenger receptor, TLR: Toll-like receptor, VEGF: Vascular endothelial growth factor.

generally less inflammatory than classically activated macrophages (Martinez et al., 2009). In analogy to the Th1 and Th2 paradigm, it was later proposed that macrophages should be termed M1 and M2 (Mills et al., 2000), representing the classically and alternatively activated macrophages, respectively.

\section{Extended nomenclature and classification of macrophage polarization}

With the observation that other stimuli promoted macrophages with immune regulatory properties that clearly differed from the alternative Th2-associated activation, an extended nomenclature was proposed that included three M2 macrophage sub-phenotypes (Mantovani et al., 2004). In this model, M2a macrophages are induced by IL-4 and IL-13 (alternatively activated), M2b are induced by immune complexes in combination with TLR ligands and M2c by IL-10 or glucocorticoids (sometimes termed deactivated). Although different in several aspects, the M2 macrophages produce low levels of proinflammatory cytokines, like IL-1 $\beta$, TNF, IL-12 and IL-23 (with the exception of M2b that produce IL-1 $\beta$, TNF and IL-6) and high levels of IL-10 and are mainly immune regulatory (Mantovani et al., 2004). Several other terms have been suggested, for instance 'innate activation' by microbial stimuli, 'humoral activation' by Fc and complement receptors and 'deactivation' by anti-inflammatory factors like IL-10, TGF- $\beta$ and glucocorticoids (Gordon, 2003), or based on their main functional properties, 'wound healing' and 'regulatory' macrophages (Mosser and Edwards, 2008). In addition, macrophages generated in vitro in the presence of GM-CSF or M-CSF alone have been described as M1 and M2 polarized, due to their pro-inflammatory versus anti-inflammatory properties (Verreck et al., 2004). Figure 2 shows a schematic view of the differences between Th1- and Th2-associated, and IL-10-induced macrophages. 
Although meant to facilitate the description of different macrophage subsets, the lack of consensus in addition to the oversimplified use and overinterpretation of some of these definitions have led to confusion and misinterpretation (reviewed in Martinez and Gordon, 2014; Murray et al., 2014). First, the phenotype and function of different subsets is mainly based on observations in vitro where a restricted number of stimuli have been used to differentiate and polarize macrophages. As a result, they do not cover the diversity of tissue-resident macrophages or macrophages associated with certain conditions or diseases, where macrophages are influenced by multiple factors that may affect macrophage polarization. In addition, in vitro macrophage subsets are commonly described as stable end-stage cells; also this in contrast to the nature of macrophages that are known to be plastic and thus able to change properties according to the surrounding microenvironment (Stout and Suttles, 2004). An additional problem is that macrophages obtained from tissues are heterogeneous in terms of phenotype, stage of differentiation and function (Biswas and Mantovani, 2010; Sica and Mantovani, 2012; Davies et al., 2013) and thus comparison with strictly defined in vitro subtypes may be misleading. In addition, the use of a restricted number of markers to define macrophage polarization has proven problematic. This is in part due to the lack of lineage-specific markers and the overlapping phenotypes and functions among macrophage subsets. One example is the use of CD206 (also known as mannose receptor, MR) as a marker of alternatively activated or M2 macrophages. Although it was originally shown to be upregulated by IL-4 and downregulated by IFN- $\gamma$ (Stein et al., 1992), studies show that GM-CSF macrophages express higher CD206 levels than M-CSF macrophages (Brocheriou et al., 2011; Kittan et al., 2013). Thus by only using CD206, GM-CSF macrophages (often defined as M1 as described above) would be classified as M2 macrophages.

In an effort to overcome the inconsistencies in macrophage definitions it was recently suggested that macrophages should be termed by the stimuli that was used to polarize them, for instance M(IL-4) or GM(IFN- $\gamma$ ) for macrophages stimulated with M-CSF and IL-4 or GM-CSF and IFN- $\gamma$, respectively (Murray et al., 2014). In addition, it was encouraged that researchers use a combination of markers to define the phenotype of the macrophages being studied. This is particularly important when describing tissue- 
derived macrophages that may not exactly fit an in vitro defined subset. In this thesis, I will use this newly described nomenclature but for simplicity I will also refer to M1 and M2 macrophages to describe macrophages with predominantly pro-inflammatory and immune regulatory properties, respectively. Importantly, M2 will not be used as a term for the originally described alternatively activated phenotype induced by $\operatorname{Th} 2$ cytokines, but as a general term for macrophages with immune suppressive or homeostatic properties.

\section{Markers differentially expressed on macrophage subtypes}

Some of the markers that have been used to define specific macrophage subpopulations (in particular M2 macrophages) in humans, are described below.

CD206 (the mannose receptor) is a pattern recognition receptor (PRR) that recognizes mannose and fucose residues on both endogenous and microbial structures (such as $M$. tuberculosis and C. albicans) leading to endocytosis and antigen presentation (Geijtenbeek and Gringhuis, 2009). CD206 was the first described marker that distinguished between macrophages stimulated by IL-4, which induced its expression, or IFN- $\gamma$, which decreased its expression (Stein et al., 1992). More recently, also GMCSF has been shown to strongly upregulate CD206 expression (Brocheriou et al., 2011; Kittan et al., 2013). CD206 has been shown to mediate anti-inflammatory responses in DCs by promoting production of IL-10, downregulation of costimulatory molecules and production of the Th2-associated chemokines CCL17 and CCL22 (Chieppa et al., 2003).

CD209 (Dendritic cell-specific intercellular adhesion molecule-3-grabbing nonintegrin, DC-SIGN) is also a PRR with specificity for mannose- and fucose-containing structures, and is expressed on immature DCs and some macrophage subsets (Geijtenbeek and Gringhuis, 2009). Expression of CD209 was initially shown to be induced by IL-4 or IL-13 and to be downregulated by lipopolysaccharide (LPS) or TNF and was therefore associated with Th2 immunity (Soilleux et al., 2002; PuigKroger et al., 2004). More recently, CD209 has been shown to be induced by M-CSF and to be expressed by IL-10-producing TAMs (Dominguez-Soto et al., 2011). In 
addition, some probiotic bacteria have been shown to utilize CD209 to reduce the production of pro-inflammatory cytokines and to induce tolerogenic DCs that promote conversion of Treg cells (Smits et al., 2005; Konstantinov et al., 2008). The fact that many pathogens also down-modulate host immune responses by targeting CD209 supports an immune regulatory role for this receptor (van Kooyk and Geijtenbeek, 2003).

The scavenger receptor CD163 is expressed on most populations of tissue-resident macrophages but not on DCs (Van den Heuvel et al., 1999) and its best characterized function is related to the clearance of senescent red blood cells (Fabriek et al., 2005). Although CD163 has mainly been identified as the receptor for hemoglobin:haptoglobin complexes, some data suggest that it may also recognize and bind bacteria and could be involved in host defense (Fabriek et al., 2009). CD163 expression is upregulated by M-CSF, IL-10 and glucocorticoids and downregulated by both Th2-associated (IL-4) and pro-inflammatory stimuli (GM-CSF, LPS, IFN- $\gamma$ and TNF) (Van den Heuvel et al., 1999; Buechler et al., 2000; Sulahian et al., 2000). Macrophages have also been shown to upregulate CD163 expression upon co-culture with Treg cells, through a mechanisms that was partly mediated by IL-10 but not Th2 cytokines (Tiemessen et al., 2007).

Neuropilin-1 (NRP-1) has been linked to Treg cells in mice (Bruder et al., 2004; Sarris et al., 2008) and has been shown to enhance the angiogenic activity of endothelial cells (Sulpice et al., 2008), implicating a role for NRP-1 in both immune suppression and tissue remodeling. In macrophages, NRP-1 expression was induced by M-CSF and suppressed by IFN- $\gamma$ and was therefore suggested as a marker of M2 macrophages (Ji et al., 2009).

Pro-inflammatory M1 macrophages are best described by their high expression of molecules associated with antigen presentation (HLA-DR, CD80/CD80 and CD40) and production of pro-inflammatory cytokines, in particular IL-12 and IL-23, and the lack of M2 markers (Mantovani et al., 2004; Biswas and Mantovani, 2010). In addition, M1 macrophages produce Th1-attracting chemokines (CXCL9, CXCL10 and CXCL11), in contrast to M2 macrophages (Th2-associated or anti-inflammatory) 
that either produce the Th2-attracting CCL17 and CCL22 or CCL2 and CCL18 (Mantovani et al., 2004; Biswas and Mantovani, 2010).

Among transcription factors, interferon regulatory factor (IRF) 5 was proposed to have a critical role in the polarization of M1 macrophages (Krausgruber et al., 2011). IRF5 was shown to be highly expressed on macrophages differentiated under the influence of GM-CSF but not M-CSF and to promote upregulation of genes encoding IL-12 and IL-23 and to repress IL-10. IRF5 ${ }^{+}$macrophages could also induce Th1 and Th17 cells but not Th2 or Treg cells and was therefore proposed as a master regulator of M1 macrophages. 


\section{Pregnancy}

\section{Establishment of the placenta}

The placenta is composed of a fetal part that includes the chorionic plate and chorionic villi, and a maternal part consisting of the endometrium (called decidua basalis) (Fig. 3 ). In the field of reproductive immunology, the fetal part of the placenta is commonly referred to as the "placenta", and the maternal part is referred to as the "decidua". Therefore, the terms "placenta" and "decidua" will be used throughout this thesis.

Already before pregnancy is established, the endometrium starts an adaptation process (decidualization) that is required for successful implantation and placental development. Decidualization occurs as part of the menstrual cycle in response to estradiol and progesterone, and involves increased vascularization, differentiation of stromal cells into decidual cells, and infiltration of leukocytes (Cartwright et al., 2010). In case that pregnancy occurs, progesterone levels are maintained high and the decidualization process continues. Implantation starts with attachment of the blastocyst to the endometrial epithelium, after which the trophoblast layer starts to differentiate into an inner cytotrophoblast layer and the surrounding multinucleated syncytiotrophoblast (Gude et al., 2004). The blastocyst sinks beneath the epithelium and becomes ultimately surrounded by the endometrium. After implantation is completed, cytotrophoblast (CTB) cells start to proliferate and differentiate, leading to the formation of the placenta with its characteristic structure with branching villi (Gude et al., 2004; Cartwright et al., 2010) (Fig. 3). Some CTB cells fuse to become the multinucleated syncytiotrophoblast layer that surrounds the floating villi. Beneath the syncytiotrophoblast is a layer of CTB cells and the villous mesenchyme, and these together form the placental membrane. This membrane functions as a barrier between maternal blood in the intervillous space and fetal blood within the capillaries in the villous core. This is also the site of oxygen and nutrient exchange and the removal of waste products. The villous mesenchyme also harbors fetal macrophages, called Hofbauer cells, which display homeostatic properties within the M2 range and may 


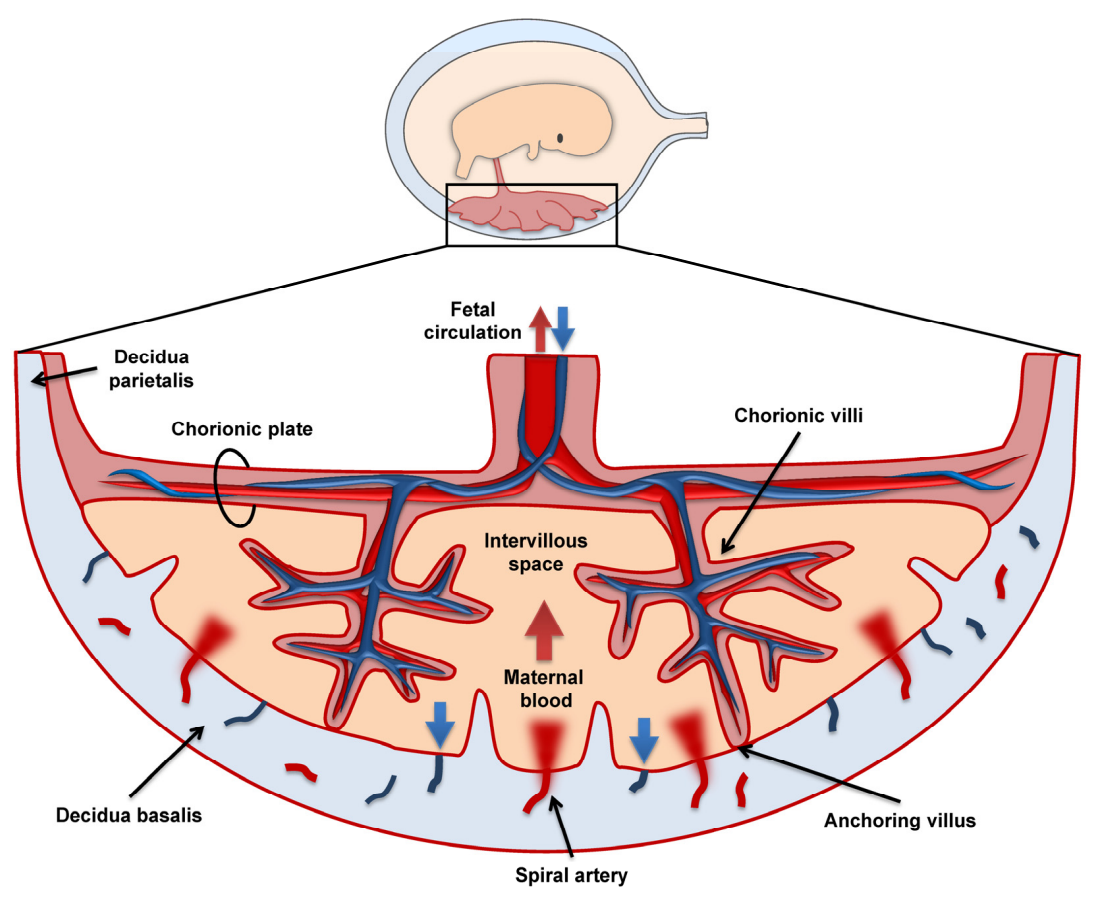

Figure 3. Schematic representation of the fully developed human placenta.

have an important phagocytic function during placental formation (Bulmer and Johnson, 1984; Bockle et al., 2008; Tang et al., 2013).

Differentiating CTB cells may also become extravillous trophoblast (EVT) cells that migrate through the anchoring villi into the decidua. EVT cells invade the decidua and migrate to remodeling vessels where they replace vascular smooth muscle cells (VSMC) and endothelial cells to form the spiral arteries. This process results in the dilation and rupture of uterine arterioles and the release of maternal blood into the intervillous space (Fig. 4, left panel). The migration of EVT cells through the decidual stroma and the remodeling of vessels is a coordinated process that involves degradation of extracellular matrix and a high rate of cell renewal and apoptosis (Cartwright et al., 2010). Decidual NK cells are considered important in this process, but decidual macrophages may also support tissue remodeling, in particular the phagocytosis of apoptotic cells (discussed later on). 


\section{Requirement of maternal immune adaptation}

The extensive invasion by trophoblast cells is a feature of haemochorial placentation, which is the most invasive type of placentation (Benirschke, 1994). The structural organization of this placental type results in several sites where maternal immune cells are exposed to trophoblast cells. The syncytiotrophoblast layer that covers the placental villi and the EVT cells that penetrate the spiral arteries are in direct contact with maternal blood leukocytes. There is also a close association between invading trophoblast cells and the leukocytes that populate the decidua (described in more detail below and in Fig. 5). Thus, although the maternal immune system is not in direct contact with the fetus itself, fetally derived trophoblast cells could potentially elicit a maternal immune response towards the semi-allogeneic fetal-placental unit. This is why the existence of mechanisms to limit maternal immune activation is of crucial importance for normal fetal development. Indeed, failure of the maternal immune system to adapt adequately during pregnancy has been associated with several pregnancy disorders. Before describing the immune adaptations that are associated with normal pregnancy, some common pregnancy complications will be introduced.

\section{Pregnancy-associated complications}

The most common pregnancy complications that may be associated with immune maladaptation are recurrent spontaneous miscarriages, preeclampsia and preterm labor.

Spontaneous miscarriage is the most common complication during early pregnancy $(\sim 15 \%)$ and is most often caused by chromosomal abnormalities or fetal malformations that are incompatible with life (Adolfsson and Larsson, 2006; Larsen et al., 2013). In contrast, recurrent spontaneous miscarriages, defined by three (sometimes two) consecutive pregnancy losses before gestational weeks 20-22, have a prevalence of $1-3 \%$ and are considered to be more heterogeneous with many possible causes (Matthiesen et al., 2012; Larsen et al., 2013). Although several risk factors 


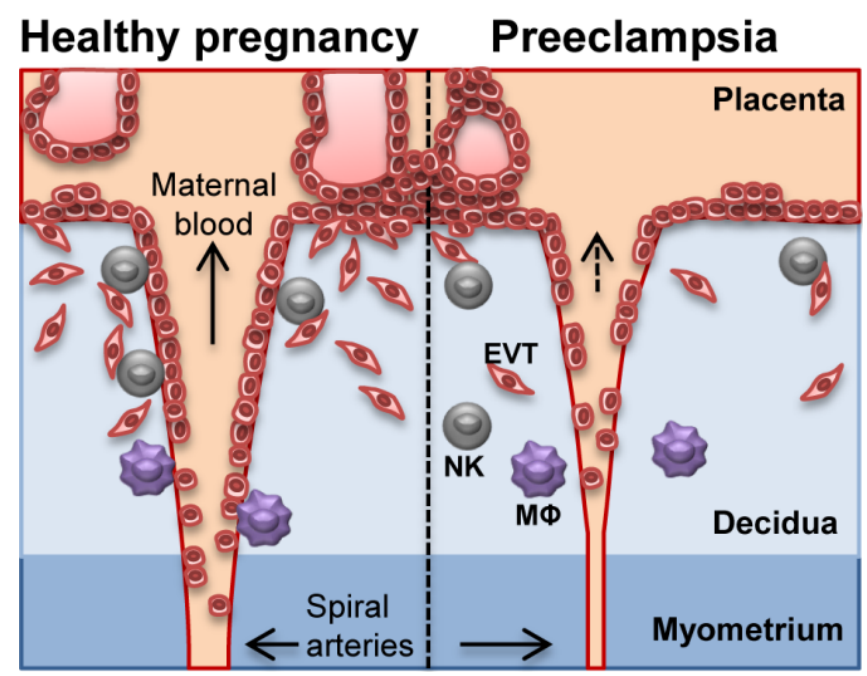

Figure 4. Spiral arteries and trophoblast invasion in healthy and preeclamptic pregnancy. Preeclampsia is characterized by shallow trophoblast invasion and defective vascular remodeling leading to reduced maternal blood flow, which in turn may compromise fetal growth. Failure of decidual natural killer (NK) cells and macrophages (MФ) to recruit trophoblast cells and support angiogenesis may contribute to the development of the disease. EVT: extravillous trophoblast. Figure from Svensson-Arvelund et al. (2014).

have been identified, including chromosomal abnormalities, endocrine dysfunction and trombophilias, about $50 \%$ of cases remain unknown (idiopathic). Increasing evidence suggests that failure of the maternal immune system to adapt properly may be an underlying cause of idiopathic recurrent miscarriage.

Preeclampsia is a disorder with multiple clinical features affecting both the mother and the fetus (Sibai et al., 2005). It is characterized by shallow trophoblast invasion and defective spiral artery remodeling (Fig. 4). The resultant poor placentation leads to reduced maternal blood flow compromising fetal growth and causing maternal hypertension. The maternal syndrome is also associated with severe inflammation and endothelial dysfunction affecting multiple organs. The pathogenesis of preeclampsia is unknown but is believed to involve maladaptation of the maternal immune system (Redman and Sargent, 2010). This concept is supported by the observations that the 
risk of developing preeclampsia is lowered with increased exposure to paternal antigens before pregnancy (Kho et al., 2009). In addition, preeclampsia occurs more often in first pregnancies, while change of partner increases the risk, supporting the hypothesis that decreased risk correlates with maternal immune adaptation to the fetus (Redman and Sargent, 2010).

Preterm birth, occurring in 5-15\% of all pregnancies, is the most common cause of neonatal deaths worldwide, and is also associated with complications later in life (Chang et al., 2013). Similar to recurrent miscarriages, preterm labor seems to be associated with multiple pathological mechanisms (Romero et al., 2014). It is generally recognized that infection-induced inflammation is the cause of a subset of all preterm deliveries (Romero et al., 2006). This is mediated by the release of inflammatory cytokines, chemokines and prostaglandins in a manner similar to spontaneous labor. However, breakdown of maternal fetal tolerance or vascular disorders (for instance preeclampsia) may also contribute to premature birth by mechanisms that are not well understood.

Thus, in spite of intensive research, the pathological mechanisms behind recurrent miscarriage, preeclampsia and preterm birth remain unresolved making prediction, prevention and treatment difficult. This may in part be due to the limited knowledge about the immune adaptations associated with healthy human pregnancy. Therefore, an increased understanding of maternal immune adaptation should offer new insights into mechanisms of potential importance also in pregnancy-associated complications. 


\section{Immune regulation during pregnancy}

\section{Characteristics of trophoblast cells}

An important property of trophoblast cells that allows interaction with maternal immune cells without triggering activation is their restricted expression of $\mathrm{MHC}$ antigens. In contrast to the fetus that has a full MHC expression, trophoblast cells completely lack MHC class II molecules as well as the most polymorphic of the classical MHC class I molecules, HLA-A and HLA-B (Trundley and Moffett, 2004). The villous syncytiotrophoblast cells that are surrounded by maternal blood are devoid of any MHC expression, while EVT cells in the decidua express the non-classical MHC class I antigens HLA-G and HLA-E, and the classical HLA-C antigen (McMaster et al., 1995; King et al., 2000a; King et al., 2000b). HLA-G has been proposed to be involved in the modulation of maternal immune responses and has for instance been shown to inhibit cytokine production and reduce NK cell cytotoxicity, to eliminate activated cytotoxic $\mathrm{CD} 8^{+} \mathrm{T}$ cells and to induce production of TGF- $\beta$ by APCs (Rieger et al., 2002; Hunt et al., 2005). Although less pronounced, HLA-E has also been shown to inhibit NK cell cytotoxicity (King et al., 2000a).

HLA-C expression may also prevent NK cell activation by binding to inhibitory NK cell receptors, and this interaction has been shown to be of particular importance for placentation, possibly by the regulation of trophoblast invasion (Hiby et al., 2004; Sanchez-Rodriguez et al., 2011). Although HLA-C could also potentially induce detrimental immune responses by $\mathrm{CD}^{+} \mathrm{T}$ cells, these cells usually do not cause fetal rejection (Tilburgs and Strominger, 2013). How this protection is mediated is not well understood, but likely involves multiple immune regulating mechanisms that are present at the fetal-maternal interface.

In addition, trophoblast cells produce large amount of hormones, including progesterone, estradiol, and human chorionic gonadotrophin (hCG) that besides promoting endocrine effects are involved in modulating the maternal immune response. Progesterone has been shown to suppress the development of Th1 cells 
while promoting Th2 cells and the production of IL-10 (Miyaura and Iwata, 2002) and to induce production of M-CSF and leukemia inhibitory factor (LIF), shown to be essential for murine pregnancy (Piccinni et al., 2001). Progesterone has also been suggested to modulate the activation of macrophages (Jones et al., 2008; Menzies et $a l .$, 2011). The effects of estradiol have been proposed to be concentration-dependent, with low concentrations promoting IFN- $\gamma$ production and Th1 immunity and high levels stimulating IL-10 secretion and Th2 immunity (Whitacre et al., 1999; Beagley and Gockel, 2003). Other effects of estradiol include reduced antigen presentation by APCs and activation of T cells and induction of Treg cells (Beagley and Gockel, 2003; Polanczyk et al., 2006). Also hCG has been shown to down-modulate immune responses by reducing the production of IFN- $\gamma$ and TNF and increasing the production of IL-10 and TGF- $\beta$ (Khil et al., 2007). In addition, hCG increased the number and suppressive function of Treg cells and prevented fetal loss in mice (Schumacher et al., 2013).

Trophoblast cells have been shown to express and produce Th2-associated and immune suppressive cytokines, including IL-4, IL-13, IL-10 and TGF- $\beta$ (Chaouat et al., 1999; Hanna et al., 2000; Sacks et al., 2001; Simpson et al., 2002). In contrast, pro-inflammatory cytokines like IL-12, TNF and IFN- $\gamma$ are present at lower levels in trophoblast cells (Sacks et al., 2001). Trophoblast cells may also be an important source of the macrophage growth factors M-CSF and GM-CSF (Bartocci et al., 1986; Jokhi et al., 1994; Engert et al., 2007). Other mechanisms that have been described to promote fetal tolerance include the elimination of maternal reactive $\mathrm{T}$ cells by indoleamine 2,3-dioxynenase (IDO) or Fas-ligand (FasL) and the induction of tolerogenic cells by for instance galectin-1 (Hunt et al., 1997; Munn et al., 1998; Blois et al., 2007).

\section{Systemic adaptations of the maternal immune system}

Despite the unique properties that trophoblast cells have acquired to limit the activation of circulating maternal immune cells, immune changes do occur systemically during pregnancy, likely as a result of placental-derived factors including 
cytokines, growth factors, hormones and trophoblast-derived microvesicles. The observations that inflammatory diseases like multiple sclerosis and rheumatoid arthritis ameliorate during pregnancy, support a systemic influence of pregnancy (Ostensen et al., 1983; Confavreux et al., 1998).

\section{Th and Treg cells}

After the initial proposal that pregnancy was associated with a shift from Th1, towards Th2 immunity (Wegmann et al., 1993), the frequencies of Th cells, including the more recently described Th17 cells, have been extensively studied in the circulation of healthy pregnant women. Despite some initial studies showing enhanced Th2 immune responses (Saito et al., 1999a), the reports have been inconsistent and the consensus appears to be that no major changes occur in the circulating Th cell compartment, and that adaptations are more likely to occur at the fetal-maternal interface (Saito et al., 2010; Ernerudh et al., 2011). Many reports have also addressed the frequency of circulating Treg cells and initial findings showed that human pregnancy was associated with increased circulating Treg cell numbers (Heikkinen et al., 2004; Sasaki et al., 2004; Somerset et al., 2004). However, the observed increase was likely due to an increase in activated non-suppressive $\mathrm{CD} 4^{+} \mathrm{CD} 25^{\text {high }} \mathrm{T}$ cells that were included when using the traditional gating strategies for Treg cells (Mjosberg et al., 2009; Ernerudh et al., 2011; Jiang et al., 2014). Most recent reports, using a more strict definition of Treg cells (for instance $\mathrm{CD} 4{ }^{\mathrm{dim}} \mathrm{CD} 25^{\text {high }}$ or including Foxp3 and CD127) show that circulating Treg cell numbers are unaltered or even decreased (Tilburgs et al., 2008; Mjosberg et al., 2009) and this has been proposed to be due to specific Treg cell migration to the decidua (Tilburgs et al., 2008).

\section{Innate immune cells}

Pregnancy has been associated with increased circulating numbers and activation of monocytes and granulocytes (Sacks et al., 1999). Granulocytes have for instance been shown to produce increased levels of CXCL8 and reactive oxygen species (Sacks et al., 1998; Luppi et al., 2002). Monocytes have been shown to upregulate the expression of activation markers like CD64 (Fc $\gamma \mathrm{RI})$, to have enhanced potential to 
produce reactive oxygen species and to produce pro-inflammatory cytokines including IL-12 and IL-1 $\beta$ (Sacks et al., 1998; Luppi et al., 2002; Sacks et al., 2003). More recent reports also show differential regulation of monocyte subsets during normal pregnancy, with increased numbers of non-classical $\left(\mathrm{CD} 14^{+/ \text {low }} \mathrm{CD} 16^{+}\right)$and decreased numbers of classical $\left(\mathrm{CD} 14^{++} \mathrm{CD} 16^{-}\right)$monocytes (Melgert et al., 2012). The nonclassical subset has been considered more pro-inflammatory due to its increased potential to produce pro-inflammatory cytokines, such as TNF and IL-1 $\beta$ (Ancuta et al., 2009; Cros et al., 2010; Ziegler-Heitbrock et al., 2010). The increased activation of blood leukocytes may in part be caused by the release of microparticles from syncytiotrophoblasts into the circulation (Germain et al., 2007). The activation of innate immune cells has been proposed to protect the mother against infections and to serve a compensatory mechanism for the weakened adaptive immunity observed in the circulation (Sacks et al., 1999). However, not all innate components show activated phenotypes; DCs have been shown to be decreased and to show a more suppressive phenotype with for instance lower expression of the co-stimulatory CD86 and increased expression of the tolerance-associated molecules CD200 and CD200R (Cordeau et al., 2012; Darmochwal-Kolarz et al., 2012). In addition, NK cells show decreased production of IFN- $\gamma$ (Veenstra van Nieuwenhoven et al., 2002) and a shift towards a Th2-associated phenotype has been proposed (Borzychowski et al., 2005). 


\section{Maternal immune adaptation at the fetal-maternal interface}

The most pronounced changes occur at the fetal-maternal interface, where maternal immune cells in the decidua are closely associated with placental trophoblast cells (Fig. 5). The human decidua is populated by a unique composition of immune cells with specialized functions that are necessary to meet the requirements associated with pregnancy. The majority of decidual leukocytes during early human pregnancy are innate immune cells, where NK cells account for $\sim 70 \%$ and macrophages $\sim 20 \%$ of all leukocytes, while only $\sim 10 \%$ are T cells (Starkey et al., 1988; King et al., 1991). Some DCs are also present $(\sim 1 \%)$, while B cells and granulocytes are scarce (Bulmer and Johnson, 1984; Gardner and Moffett, 2003; Ban et al., 2008).

\section{Restricted $T$ cell activation}

Several mechanisms have been described to limit the activation of the maternal immune system and the likelihood of mounting an anti-fetal immune response. The trophoblast cells' lack of MHC class II and classical MHC class I (HLA-A and -B) molecules (which are the main cause of $\mathrm{CD}^{+}$and $\mathrm{CD} 8^{+} \mathrm{T}$ cell activation and transplant rejection), prevents strong immune responses towards fetal antigens (Tilburgs and Strominger, 2013; Nancy and Erlebacher, 2014). Studies in mice have also shown that although decidual DCs could potentially process and present placental or fetal antigens, they fail to migrate to lymph nodes thus reducing the potential of activating Th cells (Collins et al., 2009). In addition, the recruitment of activated T cells to the decidua is limited and reactive cytotoxic T cells are eliminated by clonal deletion (Erlebacher et al., 2007; Nancy et al., 2012), thus limiting the potential of inducing an inflammatory environment in the decidua. As mentioned above, molecules produced or expressed by trophoblast cells (for instance IDO and FasL) may be in part responsible for the elimination of activated $\mathrm{T}$ cells.

\section{Th1, Th2 and Th17 cells}

Given the importance of Th cells and Treg cells in generating and controlling immune responses, much research has focused on the role of these populations in the 


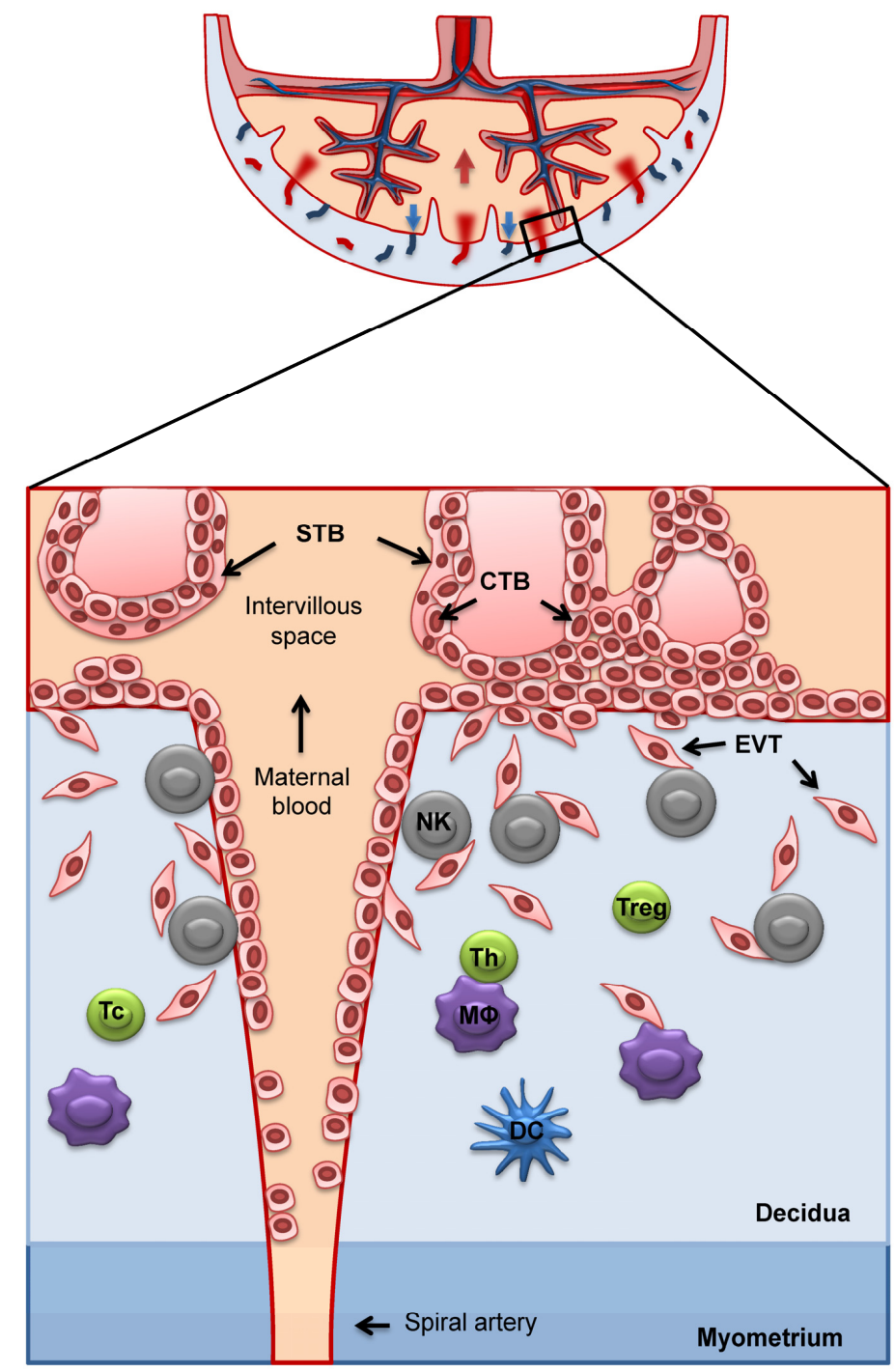

Figure 5. Schematic representation of the fetal-maternal interface. Fetal-derived trophoblast cells are in close contact with maternal immune cells in the decidua. The major leukocyte populations in the first trimester decidua are natural killer (NK) cells, macrophages (MФ), cytotoxic $\mathrm{T}(\mathrm{Tc})$ cells and $\mathrm{T}$ helper $(\mathrm{Th})$ cells, in particular regulatory $\mathrm{T}$ (Treg) cells. A small population of dendritic cells (DCs) is also present. These immune cells come into close contact with invading extravillous trophoblast cells (EVT), which proliferate from cytotrophoblast cells (CTB) and migrate from the placental villi to the decidua to take part in the remodeling of spiral arteries. STB: Syncytiotrophoblast cells. 
maintenance of human pregnancy. Early data from mouse models proposed that fetal tolerance was associated with enhanced local production of Th2 cytokines (IL-4, IL-5, IL-13 and IL-10) and that the Th1 cytokine IFN- $\gamma$ could promote fetal rejection (Lin et al., 1993; Wegmann et al., 1993; Raghupathy, 1997). Human pregnancy was initially also reported to be associated with increased IL-4 and decreased IFN- $\gamma$ secretion by decidual T cells (Saito et al., 1999b). More recent data, defining Th1, Th2 and Th17 cells by their chemokine receptor expression, showed that Th2 cells are not increased in the decidua as compared to blood and that decidual Th17 cells are scarce (Mjosberg et al., 2010). In addition, a subset of CCR6- Th1 cells that secrete moderate levels of IFN- $\gamma$ was increased, whereas $\mathrm{CCR} 6^{+}$Th1 cells associated with high secretion of IFN$\gamma$ were found to be less frequent in the early pregnancy decidua (Acosta-Rodriguez et al., 2007; Mjosberg et al., 2010). The high frequency of the CCR6 ${ }^{-}$Th1 cell subset was interpreted to be consistent with the view of early pregnancy as a state of controlled mild inflammation.

\section{Treg cells}

Treg cells have been shown to be essential to the establishment and maintenance of murine pregnancy (Aluvihare et al., 2004; Rowe et al., 2012b; Samstein et al., 2012). The accumulation of Treg cells has been shown to occur in both syngeneic and allogeneic pregnancies, and in response to fetal-specific antigens but also independently of fetal antigens, for instance by pregnancy-associated hormones (Aluvihare et al., 2004; Zhao et al., 2007; Guerin et al., 2009; Rowe et al., 2012b). In humans, $\mathrm{CD} 4^{+} \mathrm{CD} 25^{\text {high }}$ Treg cells are enriched in the decidua, express markers of activation (CD45R0 and HLA-DR) and show a suppressive phenotype, with high expression of Foxp3 and CTLA-4 (Heikkinen et al., 2004; Tilburgs et al., 2006; Tilburgs et al., 2008; Mjosberg et al., 2010; Dimova et al., 2011). In contrast to mouse pregnancy, little is known about the specificity and the mechanisms that promote expansion of Treg cells in humans. Indirect evidence suggests that human Treg cells might recognize and expand in response to paternal antigens (Mjosberg et al., 2007; Tilburgs et al., 2008). The conversion and expansion of human Treg cells may be supported by factors present in seminal fluid (in particular TGF- $\beta$ ) or by factors 
produced at the fetal-maternal interface, including hormones and cytokines (Guerin et al., 2009; Ernerudh et al., 2011; Robertson et al., 2013). It has also been suggested that the locally produced chemokines CCL4 and CCL17 attract Treg cells by binding to CCR5 and CCR4, which have been shown to be expressed on decidual Treg cells (Kallikourdis et al., 2007; Mjosberg et al., 2010). hCG may also be involved in the recruitment of Treg cells to the fetal-maternal interface in humans (Schumacher et al., 2009).

Dysregulation of Th cell populations, in particular the activation of Th1 and Th17 cells, as well as defective induction of Treg cells have been implicated in the development of pregnancy complications associated with failure to induce or maintain fetal tolerance (Saito et al., 2010; Ernerudh et al., 2011; Jiang et al., 2014).

\section{Decidual NK cells}

In peripheral blood, the majority of NK cells ( $~ 90 \%)$ belong to the cytotoxic $\mathrm{CD} 56^{\text {dim }} \mathrm{CD} 16^{\text {bright }}$ subtype, while only a small proportion belongs to the more regulatory and cytokine-secreting $\mathrm{CD} 56^{\text {bright }} \mathrm{CD} 16^{-/ \mathrm{dim}}$ subtype (Nagler et al., 1989; Cooper et al., 2001). In contrast, most human decidual NK cells are CD56 ${ }^{\text {bright }}$ CD16 (Starkey et al., 1988; King et al., 1991), and seem to be cytokine-producing cells that express immunosuppressive molecules (Koopman et al., 2003; Engert et al., 2007). In addition, although they express NK cell-activating receptors and cytolytic granules (King et al., 1991; Koopman et al., 2003; Hanna et al., 2006), decidual NK cells have been proposed to have limited cytotoxic ability (Kopcow et al., 2005), a feature that is essential for their close association with trophoblast cells. The reduced cytotoxic potential is believed to be mediated by inhibitory receptors and their interaction with MHC class I molecules (HLA-C, -E, and -G) on EVT cells (Manaster and Mandelboim, 2010; Vacca et al., 2011a).

Decidual NK cells have mostly been implicated to have a role in spiral artery remodeling. For instance, they have been shown to be closely associated with remodeling vessels (Smith et al., 2009; Hazan et al., 2010), to promote trophoblast invasion (Hanna et al., 2006; De Oliveira et al., 2010; Fraser et al., 2012) and to 
stimulate vascular remodeling by the production of angiogenic factors (Hanna et al., 2006; Lash et al., 2006; Kalkunte et al., 2009). The interaction of activating NK cell receptors with MHC I molecules on trophoblast cells seems to promote both chemotactic and angiogenic factors (Hanna et al., 2006), suggesting that a moderate level of activation is necessary for proper trophoblast invasion and spiral artery remodeling. The importance of this balance has been demonstrated by studies showing that too much inhibition by NK cell receptors is associated with an increased risk of developing preeclampsia (Hiby et al., 2004; Sanchez-Rodriguez et al., 2011), which is characterized by poor trophoblast invasion and impaired vascular remodeling.

The origin of decidual NK cells is not well established but may involve the maturation from endometrial NK cells or hematopoietic precursors in response to pregnancyassociated factors (Manaster et al., 2008; Vacca et al., 2011b). In addition, NK cells might be recruited from peripheral blood and differentiate locally into decidual NK cells, for instance in response to TGF- $\beta$ (Keskin et al., 2007; Carlino et al., 2008; Male et al., 2010). The recruitment from blood to decidua may be mediated by CXCL10 and CXCL12 through the interaction with CXCR3 and CXCR4 that have been shown to be expressed on decidual NK cells (Hanna et al., 2003).

\section{Decidual macrophages}

Macrophages represent the most abundant APC population in the decidua and their high frequency persists throughout pregnancy (Bulmer and Johnson, 1984; Starkey et al., 1988; Williams et al., 2009). Given their potential to respond to microbial antigens as well as alloantigens, decidual macrophages have been proposed to play a central role in creating a balance between the activation of pro-inflammatory responses and the maintenance of immune homeostasis during pregnancy (Nagamatsu and Schust, 2010b; Svensson-Arvelund and Ernerudh, 2015). Furthermore, due to their proinflammatory potential, decidual macrophages have also been implicated in the initiation of parturition, which is characterized by production of inflammatory cytokines, including IL-1 $\beta$, IL-6 and TNF, and infiltration of leukocytes with 
inflammatory properties (Nagamatsu and Schust, 2010a; Hamilton et al., 2013). However, the most important immunological events occur in early pregnancy, the time when the placenta is being established and when the maternal immune system needs to adjust to support the growing fetus. Therefore, literature reporting the phenotype and function of macrophages from early human pregnancy will be described below (if not otherwise stated).

\section{Phenotype and function of decidual macrophages}

Two decades ago it was shown that decidual macrophages from early human pregnancy had a more suppressive effect on the proliferation of $\mathrm{T}$ cells than blood monocytes in a mixed lymphocyte reaction (Mizuno et al., 1994). This study suggested that decidual macrophages have the ability to process and present alloantigens and possess immune suppressive activity. Besides this early observation, the function of decidual macrophages has mainly been deduced from gene expression, cell surface phenotyping and cytokine secretion analyses. For instance, the observation that decidual macrophages were the major source of IL-10 among decidual leukocytes, implicated them as important immune suppressors (Lidstrom et al., 2003). At the same time, it was reported that the majority of $\mathrm{CD} 14^{+}$decidual macrophages expressed the pattern recognition receptors CD209 (DC-SIGN) and CD206 (mannose receptor) associated with an M2 macrophage phenotype (Kammerer et al., 2003; Laskarin et al., 2005). Further, global gene expression profiling showed that a large number of upregulated genes in decidual macrophages were associated with immune regulation and M2 polarization (Gustafsson et al., 2008). These included the previously described CD206 and CD209, but also the newly proposed M2 marker NRP-1 and the chemokines CCL2 and CCL18. Another observation indicating an immune regulatory function of decidual macrophages was their role in the clearance of apoptotic cells (Mor and Abrahams, 2003). It is only recently that the function of human decidual macrophages has been studied in more detail, and several reports now suggest that decidual macrophages influence the function of both decidual NK cells and T cells (Vacca et al., 2010; Co et al., 2013; Sayama et al., 2013). The function of 


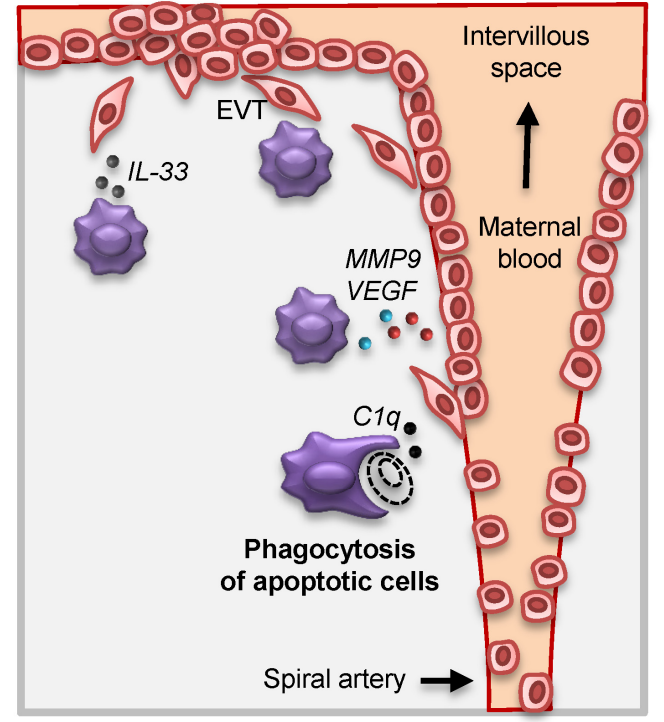

Figure 6. Decidual macrophages may support spiral artery remodeling by promoting trophoblast invasion (for instance by IL-33) and producing factors associated with tissue remodeling and angiogenesis (MMP9 and VEGF). They may also maintain a homeostatic tissue environment by phagocytosing apoptotic cells and debris. EVT: Extravillous trophoblast, MMP: Matrix metalloproteinase, VEGF: Vascular endothelial growth factor. Figure adapted from Svensson-Arvelund and Ernerudh (2015).

decidual macrophages is likely controlled by epigenetic mechanisms; in particular, genes encoding M1-associated factors (for instance IL-1 $\beta$ and the IL-12 receptor $\beta 2$ subunit) are hypermethylated, while genes encoding M2-associated factors (for instance A2M and IL-10) are hypomethylated, suggesting a predisposition towards an immune regulatory phenotype (Kim et al., 2012).

Decidual macrophages may also have other functions, an area of research that was in part reinforced by microarray data reveling expression of tissue remodeling genes (Gustafsson et al., 2008). For instance, decidual macrophages contribute to spiral artery remodeling by promoting trophoblast invasion into the decidua and by remodeling of the extracellular matrix (Fig. 6). This concept is supported by the accumulation of decidual macrophages in areas close to invading trophoblast cells and remodeling spiral arteries (Smith et al., 2009; Helige et al., 2014). Decidual macrophages express or produce factors particularly associated with tissue remodeling, for instance matrix metalloproteinase-9, fibronectin-1 and collagens, and angiogenic factors such as epidermal growth factor (EGF) and vascular endothelial growth factor (VEGF) (Engert et al., 2007; Li et al., 2009; Smith et al., 2009). In 
addition, decidual macrophages produce chemotactic cytokines and chemokines (for instance CXCL8 and IL-33) proposed to promote trophoblast recruitment (Engert et al., 2007; Fock et al., 2013). Another important function attributed to decidual macrophages is the clearance of debris and apoptotic cells that are generated during spiral remodeling. Supporting this, macrophages with phagocytic activity are closely associated with apoptotic VSMCs around the remodeling vessels (Hazan et al., 2010), and production of molecules such as fibronectin-1 and C1q by decidual macrophages may facilitate the uptake of apoptotic cells (Gustafsson et al., 2008; SvenssonArvelund and Ernerudh, 2015). The process of apoptotic clearance may be particularly important to limit the inflammatory potential at the fetal-maternal interface (Mor and Abrahams, 2003; Svensson-Arvelund and Ernerudh, 2015).

\section{Origin and development of decidual macrophages}

The origin of decidual macrophages in human pregnancy is largely unknown. Experiments in mice suggest that uterine macrophages originate from blood-derived Ly6 $\mathrm{C}^{\text {hi }}$ monocytes, the mouse counterpart of classical CD $14^{++} \mathrm{CD} 16^{-}$human monocytes, by a CCR2-dependent mechanism (Tagliani et al., 2011). CCL2, the main CCR2 ligand, is produced by many cells at the fetal-maternal interface in humans, including macrophages, NK cells, decidual stromal and trophoblast cells (Engert et al., 2007; Gustafsson et al., 2008; He et al., 2012), suggesting that macrophage numbers may be maintained by the active recruitment of blood monocytes, in a manner similar to mouse pregnancy. The high proportion of proliferating $\left(\mathrm{Ki} 67^{+}\right)$ decidual macrophages also suggests that they proliferate on site, possibly from endometrial macrophages, which represent one of the major leukocyte populations in the non-pregnant uterus (Kammerer et al., 2003). This idea would support the recently observed importance of macrophage proliferation in most tissues (Hashimoto et al., 2013). At least in the mouse uterus, macrophage proliferation was shown to be induced by M-CSF (Tagliani et al., 2011). However, very little research has focused on the mechanisms that control human decidual macrophage differentiation and function, and the factors that influence the polarization of these cells are unknown. 


\section{The role of decidual macrophages in pregnancy complications}

Given their important role in both immune regulation and tissue remodeling, macrophages have been implicated in the development of pregnancy complications. Most studies have focused on the number of decidual macrophages and these have failed to show consistent results for instance showing both unaltered, decreased and increased numbers of macrophages in preeclamptic patients (Burk et al., 2001; Lockwood et al., 2006; Kim et al., 2007; Schonkeren et al., 2011). Thus, the role of decidual macrophages in the development of complications is likely to be more related to the function of macrophages (or a combination of the both). Indeed, some studies have reported altered expression of M2 macrophage markers (for instance CD163, CD206 and CD209) (Schonkeren et al., 2011; Hsu et al., 2012; Prins et al., 2012) or production of factors influencing macrophage polarization (for instance GM-CSF and IL-10) (Hennessy et al., 1999; Huang et al., 2010; Schonkeren et al., 2011) in pregnancy complications. However, evidence is still scarce, partly reflecting the limited information about decidual macrophage function during healthy pregnancy. 


\section{Aims and hypotheses}

The general aim of this thesis was to increase our understanding of how the maternal immune system adapts during pregnancy to accept the semi-allogeneic fetus. The adaptations that occur at the fetal-maternal interface were assessed, in particular the role of decidual macrophages in maintaining a tolerogenic microenvironment but also the role of the placenta in promoting immune cells with regulatory properties.

\section{Specific aims}

- The aim of paper I was to characterize first trimester decidual macrophages in terms of cell surface expression and secretion of cytokines, and to identify key molecules involved in the polarization of decidual macrophages into cells with an immunosuppressive phenotype.

- The aim of paper II was to analyze the immunological function of decidual macrophages, in particular their role in promoting the unique composition and phenotype of decidual leukocytes.

- The aim of paper III was to investigate if the placenta could contribute to the tolerant immune environment that is necessary for protecting both itself and the fetus. More specifically, we tested the ability of human placental tissue and trophoblast cells from early human pregnancy to induce homeostatic M2 macrophages and Treg cells and searched for specific factors involved in this process. 


\section{Hypotheses}

- In paper I we hypothesized that decidual macrophages would show a phenotype characteristic for M2 macrophages and that this phenotype could be induced by anti-inflammatory (IL-10) or Th2-related (IL-4 and IL-13) cytokines or by pregnancy-associated hormones (in particular progesterone and estradiol).

- In paper II we hypothesized that decidual macrophages would mainly produce immunosuppressive cytokines and induce Treg cells. We also hypothesized that decidual macrophages would produce chemokines associated with the recruitment of NK cells, monocytes and Treg cells. Further, we anticipated that the cytokine and chemokine profile of decidual macrophages would be similar to that of macrophages polarized with M-CSF and IL-10.

- In paper III we hypothesized that the fetal placenta, being a temporary organ closely associated with maternal leukocytes, would promote the polarization of homeostatic M2 macrophages and the induction of Treg cells. In particular, placental trophoblast cells were expected to play a major role in the adaptation process, through production of molecules such as M-CSF, IL-10, TGF- $\beta$, LIF and CCL18. 


\section{Study design and methodological considerations}

\section{Subjects}

A total of 66 healthy first trimester pregnant women undergoing elective surgical abortions at Linköping University Hospital, Sweden $(n=50)$ or at the Royal Victoria Infirmary, Newcastle upon Tyne, UK $(n=16)$ were included in papers I-III. The maternal age and gestational length of the pregnant women in papers I-III is summarized in Table I. In all cases, pregnancies were viable as determined by crownrump length by using ultrasound. Misoprostol (Cytotec $\left.{ }^{\circledR}\right)$ was given to all women prior to surgery. In all studies, samples were collected after obtaining informed consent and the studies were approved by the Local Ethics Committees of Linköping University (papers I-III) and Newcastle and North Tyneside (paper III).

In paper I, decidual tissues and venous blood samples were collected from 28 of the pregnant women recruited in Linköping (Table II). In paper II, decidual tissues and venous blood samples were collected from 17 of the pregnant women recruited in Linköping. Eleven of these women were also included in paper I.

Table I. Information about the pregnant women participating in papers I-III*

\begin{tabular}{cccc} 
& $\begin{array}{c}\text { Paper I } \\
(\mathbf{n}=\mathbf{2 8})\end{array}$ & $\begin{array}{c}\text { Paper II } \\
(\mathbf{n = 1 7})\end{array}$ & $\begin{array}{c}\text { Paper III } \\
(\mathbf{n = 4 5})\end{array}$ \\
\hline \hline $\begin{array}{c}\text { Age at inclusion } \\
\text { (years) }\end{array}$ & $28(17-43)$ & $32(20-43)$ & $25(16-42)$ \\
$\begin{array}{c}\text { Gestational age at } \\
\text { inclusion (weeks) }\end{array}$ & $10(7-11)$ & $9(7-11)$ & $9(7-11)$ \\
\hline
\end{tabular}

*Data is shown as median and range (parentheses). 
Table II. Overview of patient samples used in papers I-III

\begin{tabular}{|c|c|c|c|c|c|}
\hline \multirow[b]{2}{*}{ Patient ID } & \multicolumn{2}{|c|}{ Paper I } & \multirow{2}{*}{$\begin{array}{l}\text { Paper II } \\
\text { Isolated } \\
\text { DecMФ }\end{array}$} & \multicolumn{2}{|c|}{ Paper III } \\
\hline & DMC & $\begin{array}{l}\text { Isolated } \\
\text { DecMФ }\end{array}$ & & $\begin{array}{l}\text { Placental } \\
\text { tissue }\end{array}$ & $\begin{array}{c}\text { Isolated } \\
\text { CTB/EVT cells }\end{array}$ \\
\hline Dec\#3 & & & & $\mathrm{x}$ & \\
\hline Dec\#10 & $\mathrm{x}$ & & & & \\
\hline Dec\#11 & $\mathrm{x}$ & & & & \\
\hline Dec\#12 & $\mathrm{x}$ & & & & \\
\hline Dec\#15 & $\mathrm{x}$ & & & & \\
\hline Dec\#16 & $\mathrm{x}$ & & & & \\
\hline Dec\#17 & $\mathrm{x}$ & & & $\mathrm{x}$ & \\
\hline Dec\#19 & $\mathrm{x}$ & & & & \\
\hline Dec\#20 & $\mathrm{x}$ & & & & \\
\hline Dec\#21 & $\mathrm{x}$ & & & & \\
\hline Dec\#23 & $\mathrm{x}$ & & & & \\
\hline Dec\#24 & $\mathrm{x}$ & & & & \\
\hline Dec\#25 & $\mathrm{x}$ & & & & \\
\hline Dec\#26 & $\mathrm{x}$ & & & $\mathrm{x}$ & \\
\hline Dec\#28 & $\mathrm{x}$ & & & & \\
\hline Dec\#30 & $\mathrm{x}$ & & & & \\
\hline Dec\#35 & $\mathrm{x}$ & & & & \\
\hline Dec\#36 & $\mathrm{x}$ & $\mathrm{x}$ & $\mathrm{x}$ & $\mathrm{x}$ & \\
\hline Dec\#37 & & $\mathrm{x}$ & $\mathrm{x}$ & $\mathrm{x}$ & \\
\hline Dec\#38 & & $\mathrm{x}$ & $\mathrm{x}$ & & \\
\hline Dec\#39 & & $\mathrm{x}$ & $\mathrm{x}$ & $\mathrm{x}$ & \\
\hline Dec\#41 & & $\mathrm{x}$ & $\mathrm{x}$ & & \\
\hline Dec\#42 & & $\mathrm{x}$ & $\mathrm{x}$ & & \\
\hline Dec\#43 & & $\mathrm{x}$ & $\mathrm{x}$ & $\mathrm{x}$ & \\
\hline Dec\#44 & $\mathrm{x}$ & $\mathrm{x}$ & $\mathrm{x}$ & & \\
\hline Dec\#45 & $\mathrm{x}$ & & & & \\
\hline Dec\#46 & $\mathrm{x}$ & $\mathrm{x}$ & $\mathrm{x}$ & $\mathrm{x}$ & \\
\hline Dec\#47 & & $\mathrm{x}$ & $\mathrm{x}$ & $\mathrm{x}$ & \\
\hline Dec\#48 & $\mathrm{x}$ & $\mathrm{x}$ & $\mathrm{x}$ & $\mathrm{x}$ & \\
\hline Dec\#49 & & & & $\mathrm{x}$ & \\
\hline Dec\#54 & & & & $\mathrm{x}$ & \\
\hline Dec\#55 & & & & $\mathrm{x}$ & \\
\hline Dec\#57 & & & & $\mathrm{x}$ & \\
\hline Dec\#58 & & & $\mathrm{x}$ & $\mathrm{x}$ & \\
\hline Dec\#59 & & & & $\mathrm{x}$ & \\
\hline Dec\#60 & & & & $\mathrm{x}$ & \\
\hline Dec\#61 & & & & $\mathrm{x}$ & \\
\hline Dec\#62 & & & & $\mathrm{x}$ & \\
\hline Dec\#63 & & & & $\mathrm{x}$ & \\
\hline Dec\#64 & & & & $\mathrm{x}$ & \\
\hline Dec\#65 & & & & $\mathrm{x}$ & \\
\hline Dec\#67 & & & $\mathrm{x}$ & $\mathrm{x}$ & \\
\hline Dec\#68 & & & & $\mathrm{x}$ & \\
\hline
\end{tabular}


Table II. Cont.

\begin{tabular}{|c|c|c|c|c|c|}
\hline & \multicolumn{2}{|c|}{ Paper I } & \multirow{2}{*}{$\begin{array}{l}\text { Paper II } \\
\text { Isolated } \\
\text { DecMФ }\end{array}$} & \multicolumn{2}{|c|}{ Paper III } \\
\hline Patient ID & DMC & $\begin{array}{l}\text { Isolated } \\
\text { DecMФ }\end{array}$ & & $\begin{array}{c}\text { Placental } \\
\text { tissue }\end{array}$ & $\begin{array}{c}\text { Isolated } \\
\text { CTB/EVT cells }\end{array}$ \\
\hline Dec\#69 & & & & $\mathrm{x}$ & \\
\hline Dec\#70 & & & & $\mathrm{x}$ & \\
\hline Dec\#71 & & & & $\mathrm{x}$ & \\
\hline Dec\#72 & & & $\mathrm{x}$ & $\mathrm{x}$ & \\
\hline Dec\#73 & & & $\mathrm{x}$ & $\mathrm{x}$ & \\
\hline Dec\#76 & & & $\mathrm{x}$ & & \\
\hline Dec\#78 & & & $\mathrm{x}$ & & \\
\hline B1303 & & & & & $\mathrm{x}$ \\
\hline B1307 & & & & & $\mathrm{x}$ \\
\hline B1308 & & & & & $\mathrm{x}$ \\
\hline B1309 & & & & & $\mathrm{x}$ \\
\hline B1312 & & & & & $\mathrm{x}$ \\
\hline B1313 & & & & & $\mathrm{x}$ \\
\hline B1317 & & & & & $\mathrm{x}$ \\
\hline B1318 & & & & & $\mathrm{x}$ \\
\hline B1320 & & & & & $\mathrm{x}$ \\
\hline B1321 & & & & & $\mathrm{x}$ \\
\hline B1324 & & & & & $\mathrm{x}$ \\
\hline B1325 & & & & & $\mathrm{x}$ \\
\hline B1327 & & & & & $\mathrm{x}$ \\
\hline B1328 & & & & & $\mathrm{x}$ \\
\hline B1330 & & & & & $\mathrm{x}$ \\
\hline B1331 & & & & & $\mathrm{x}$ \\
\hline
\end{tabular}

DMC: Decidual mononuclear cells, DecMФ: Decidual macrophages, CTB: Cytotrophoblast, EVT: Extravillous trophoblast.

In paper III, first trimester placental tissues were collected from 45 pregnant women, 29 recruited in Linköping and 16 in Newcastle. Thirteen of the women from Linköping were also included in paper I or II. In papers I and II, decidual cells were analyzed, while only placental tissue was used in paper III. Samples from the women recruited in Newcastle, were exclusively used to isolate trophoblast cells (Table II).

For the in vitro assays in papers I-III, blood samples were collected on one or more occasions from healthy non-pregnant female volunteers not taking hormonal contraceptives or any other medication. Twelve different women are included in paper I, 17 in paper II and 23 in paper III. 


\section{Ethical considerations}

The use of samples from first trimester women undergoing elective abortions raises some ethical concerns. An important aspect is the use of abortion material; in our studies, we only use tissues from the mother (decidua) and placenta and not the fetus itself. The aborted fetus is treated with respect and according to the general guidelines for aborted material at the clinic. Another ethical consideration is whether it is ethically correct to ask women for participation when they are in a vulnerable situation. Here, the first judgement is done by the medical staff who will not ask women for participation when this is considered inappropriate. The women are then always given oral and written information about the study, which is done after the decision of the abortion procedure has been made. Participation is always voluntary and it is made clear that participation will not affect the present or future treatment and that the decision can be withdrawn at any time. Since the information is usually given a couple of weeks before the procedure, the women have time to consider their decision without feeling pressure. Also, most women agree to participate as they feel they contribute to a good thing.

\section{First trimester samples as a model of healthy pregnancy}

In papers I-III, samples obtained from human first trimester pregnancy terminations were used to study immune adaptations associated with healthy pregnancy. One major caveat of this model is the fact that the outcome of these specific pregnancies is unknown. That is, despite showing normal development at the time of ultrasound before the surgical procedure, it is possible that some of the pregnancies might have developed complications later on. Nevertheless, the majority of pregnancies are likely to have developed normally meaning that results obtained from these samples mostly represent the normal situation. In addition, pregnancies that may have developed complications are likely to be heterogeneous with varying underlying causes, and the impact on a specific analysis should be limited. 
Recently described approaches may help overcome this problem. For instance, first trimester decidual tissue could be obtained during chorionic villous sampling and the results could therefore be correlated with the outcome of pregnancy (Prins et al., 2012). One limitation of this approach is the small amount of tissue that can be obtained, in addition to the limited number and perhaps selected set of patients that may undergo chorionic villous sampling. Another method may be the use of uterine artery Doppler ultrasound to define first trimester pregnancies with normal or high resistance indices. This measurement reflects the level of spiral artery remodeling and thus the risk of developing complications associated with impaired vascular remodeling, such as preeclampsia (Fraser et al., 2012). However, only an approximation of the risk is obtained and only to a certain group of complications.

All women undergoing surgical pregnancy terminations were given the synthetic prostaglandin E1 (PGE1) analogue misoprostol (administered orally or vaginally), which is used to enhance uterine contractility and cervical ripening (Tang et al., 2007). However, misoprostol has also been shown to influence the local immune response by promoting the infiltration of monocytes and neutrophils to the decidua after vaginal treatment (Milne et al., 2005). In contrast, misoprostol administrated orally reduced the proliferation of peripheral blood mononuclear cells (PBMC) and inhibited production of IFN- $\gamma$, IL-2 and IL-4 but did not affect IL-10 production (Waiser et al., 2003). Increased phagocytosis by PBMC was also observed, but the phagocytosing leukocyte population was not identified in this study. Although the effects of in vivo administrated misoprostol on human macrophages have not been described, monocytes stimulated in vitro with PGE1 show decreased expression of the co-stimulatory molecules CD86 and CD40 (Takahashi et al., 2003). Misoprostol or PGE1 have also inhibitory effects on the inflammatory cytokines IL-18 and TNF by monocytes and macrophages; however, these effects were observed upon LPSstimulated cytokine production and the spontaneous effects appear to be modest (Haynes et al., 1992; Takahashi et al., 2005). The effects of misoprostol on PRRs, such as CD163, CD206 or CD209, have to our knowledge not been described. 
It can thus not be excluded that misoprostol treatment may influence both the number and phenotype of decidual macrophages. In theory, monocyte infiltration would result in a higher percentage of newly arrived monocytes in relation to fully differentiated decidual macrophages, and in that case the proportion of mature macrophages expressing certain markers may be underestimated in our results. Regarding the effect on macrophage function, it is possible that misoprostol treatment may have skewed macrophages towards a more anti-inflammatory phenotype. However, since plasma concentrations of misoprostol reach similar levels within 2 hours, regardless of the route of administration (Tang et al., 2007), blood monocytes would also have been affected by this treatment. Comparison between blood monocytes from pregnant and non-pregnant women, suggests that misoprostol has no major impact at least on the phenotype of blood monocytes. Also, the potential impact of misoprostol on the interpretation of macrophage function is reduced when comparing decidual macrophages with blood monocytes or by using monocytes as baseline, as is the case for microarray data. 


\section{Experimental design}

In the following sections, the design of experiments used in papers I-III will be described and discussed. An overview of the analytical methods used in papers I-III is shown in Table III; and a summary of the analyzed markers, cytokines and chemokines is shown in Table IV; details are described in the respective papers.

Table III. Overview of the methods used in papers I-III

\begin{tabular}{|c|c|c|c|}
\hline & Paper I & Paper II & Paper III \\
\hline \multicolumn{4}{|l|}{ MACS } \\
\hline Blood CD14 $4^{+}$ & $\mathrm{x}$ & $\mathrm{x}$ & $\mathrm{x}$ \\
\hline Blood $\mathrm{CD}^{+}$ & & $\mathrm{x}$ & $\mathrm{x}$ \\
\hline Decidua CD14 ${ }^{+}$ & $\mathrm{x}$ & $\mathrm{x}$ & \\
\hline \multicolumn{4}{|l|}{ FACS } \\
\hline Treg and responder Th cells & & & $\mathrm{x}$ \\
\hline Flow cytometry & $\mathrm{x}$ & $\mathrm{x}$ & $\mathrm{x}$ \\
\hline Multiplexed bead assay & $\mathrm{x}$ & $\mathrm{x}$ & $\mathrm{x}$ \\
\hline ELISA & & $\mathrm{x}$ & $\mathrm{x}$ \\
\hline Real-time RT-PCR & $\mathrm{x}$ & $\mathrm{x}$ & $\mathrm{x}$ \\
\hline Microarray & $\mathrm{x}$ & $\mathrm{x}$ & \\
\hline Immunohistochemistry & & & $\mathrm{x}$ \\
\hline
\end{tabular}

\section{In vitro polarization of macrophages}

In papers I and II, macrophages generated in vitro were analyzed in parallel with decidual macrophages. Briefly, $\mathrm{CD} 14^{+}$monocytes isolated from peripheral blood were cultured for six days in culture medium containing $10 \%$ fetal calf serum and either GM-CSF or M-CSF and additional polarizing factors (Fig. 7). One of the major objectives was to identify factors that regulate the differentiation and polarization of decidual macrophages (including growth factors, but also cytokines and hormones). Therefore, it would have been desirable to culture macrophages also in the absence of M-CSF or GM-CSF, which alone promote macrophages with distinct functional 
Table IV. Markers, cytokines and chemokines analyzed in papers I-III

\begin{tabular}{|c|c|c|c|c|}
\hline & Method & $\begin{array}{l}\text { Paper } \\
\text { I }\end{array}$ & $\begin{array}{l}\text { Paper } \\
\text { II }\end{array}$ & $\begin{array}{c}\text { Paper } \\
\text { III }\end{array}$ \\
\hline \multicolumn{5}{|l|}{ Monocytes/Macrophages } \\
\hline $\begin{array}{l}\text { CD14, ICAM-3, CD163, } \\
\text { CD206, CD209 }\end{array}$ & Flow cytometry & $\mathrm{x}$ & & $\mathrm{x}$ \\
\hline CD11c, NRP-1 & Flow cytometry & $\mathrm{x}$ & & \\
\hline IRF5 & Real-time RT-PCR & $\mathrm{x}$ & & \\
\hline *IL-10 & $\begin{array}{l}\text { Multiplexed bead } \\
\text { assay }\end{array}$ & $\mathrm{x}$ & & $\mathrm{x}$ \\
\hline$*$ IL-1 $\beta$, IL-6, TNF, CCL4 & $\begin{array}{l}\text { Multiplexed bead } \\
\text { assay }\end{array}$ & $\mathrm{x}$ & & \\
\hline *IL-12p70, IL-23 & $\begin{array}{l}\text { Multiplexed bead } \\
\text { assay }\end{array}$ & & $\mathrm{x}$ & $\mathrm{x}$ \\
\hline$*$ IL-27 & $\begin{array}{l}\text { Multiplexed bead } \\
\text { assay }\end{array}$ & & $\mathrm{x}$ & \\
\hline$*$ IL-35 & ELISA & & $\mathrm{x}$ & \\
\hline TGF- $\beta 1-3$ & $\begin{array}{l}\text { Multiplexed bead } \\
\text { assay }\end{array}$ & & & $\mathrm{x}$ \\
\hline$* p 35, p 19, p 40, E b i 3, p 28$ & Real-time RT-PCR & & $\mathrm{x}$ & \\
\hline $\begin{array}{l}\text { *CXCL1-3, CCL2, CXCL10, } \\
\text { CCL22, CCL20 }\end{array}$ & $\begin{array}{l}\text { Multiplexed bead } \\
\text { assay }\end{array}$ & & $\mathrm{x}$ & \\
\hline *CCL18 & ELISA & & $\mathrm{x}$ & $\mathrm{x}$ \\
\hline $\begin{array}{l}420 \text { macrophage-related genes } \\
\text { (100 decidual macrophage- } \\
\text { specific) }\end{array}$ & Microarray & $\mathrm{x}$ & & \\
\hline $\begin{array}{l}589 \text { Immune Response genes, } 40 \\
\text { with Chemotactic Activity }\end{array}$ & Microarray & & $\mathrm{x}$ & \\
\hline \multicolumn{5}{|l|}{ Th cells } \\
\hline $\mathrm{CD} 4, \mathrm{CD} 25$ & Flow cytometry & & $\mathrm{x}$ & $\mathrm{x}$ \\
\hline $\begin{array}{l}\text { CD127, CD39, CTLA-4, } \\
\text { CD45R0, CD45RA, HLA-DR, } \\
\text { CD69 }\end{array}$ & Flow cytometry & & & $\mathrm{x}$ \\
\hline Foxp3, T-bet, GATA-3, Roryt & Flow cytometry & & $\mathrm{x}$ & $\mathrm{x}$ \\
\hline Foxp3 & Real-time RT-PCR & & & $\mathrm{x}$ \\
\hline $\begin{array}{l}\text { GM-CSF, IL-2, IL-10, IL-13, } \\
\text { IL-17, IFN- } \gamma, \text { TGF- } \beta 1-3\end{array}$ & $\begin{array}{l}\text { Multiplexed bead } \\
\text { assay }\end{array}$ & & & $\mathrm{x}$ \\
\hline \multicolumn{5}{|l|}{ PBMC (Transwell system) } \\
\hline $\begin{array}{l}\text { CD14, CD3, CD4, CD8, CD56, } \\
\text { CD25, CD19 }\end{array}$ & Flow cytometry & & $\mathrm{x}$ & \\
\hline
\end{tabular}


Table IV. Cont.

\begin{tabular}{|c|c|c|c|c|}
\hline & Method & $\begin{array}{c}\text { Paper } \\
\text { I }\end{array}$ & $\begin{array}{c}\text { Paper } \\
\text { II }\end{array}$ & $\begin{array}{c}\text { Paper } \\
\text { III }\end{array}$ \\
\hline \multicolumn{5}{|l|}{ Placenta/trophoblast cells } \\
\hline $\begin{array}{l}\text { GM-CSF, M-CSF, IL-10, TGF- } \\
\beta 1-3, \text { IL-1RA, IL-1 } \beta, \text { IL-6, TNF, } \\
\text { IL-12p70, IL-23, IFN- } \gamma \text {, IL-13, } \\
\text { IL-17, IL-2, TRAIL, IL-33, LIF, } \\
\text { TSLP, CCL2, CXCL1-3, } \\
\text { CXCL8, CXCL10, CXCL11, } \\
\text { CCL17, CCL20, CCL22 }\end{array}$ & $\begin{array}{l}\text { Multiplexed bead } \\
\text { assay }\end{array}$ & & & $\mathrm{x}$ \\
\hline CCL18 & ELISA & & & $\mathrm{x}$ \\
\hline $\begin{array}{l}\text { M-CSF, GM-CSF, IL-10, CD14, } \\
\text { CD163 }\end{array}$ & $\begin{array}{c}\text { Immuno- } \\
\text { histochemistry }\end{array}$ & & & $\mathrm{x}$ \\
\hline
\end{tabular}

*Also analyzed in CD14-depleted decidual and blood mononuclear cells

properties (M2- and M1-like, respectively). Although the presence of colonystimulating factors is essential for macrophage survival and differentiation in vitro, it has been shown that human serum alone may be sufficient to promote macrophage survival or differentiation (Andreesen et al., 1990; Brugger et al., 1991) and could therefore potentially be used to differentiate "unpolarized" macrophages. However, we found that macrophages cultured in the presence of human serum without addition of growth factors showed markedly reduced viability and differentiated poorly (data not shown). Having to choose growth factor, it could be argued that M-CSF would be the optimal choice. Due to its constitutive production in most tissues and the high circulating levels it has been proposed that exposure to M-CSF should be viewed as the normal condition for differentiating macrophages (Hamilton, 2008). M-CSF is also the predominant growth factor used to differentiate macrophages in vitro (Murray et al., 2014). In contrast, GM-CSF controls DC homeostasis in vivo (Greter et al., 2012a) and is commonly used to generate monocyte-derived DCs, usually in combination with IL-4 or IL-13 (Conti and Gessani, 2008). However, although most tissue macrophage populations are dependent on M-CSF, it should be remembered that some macrophages, such as alveolar macrophages and microglia, are rather dependent on GM-CSF (or IL-34). 


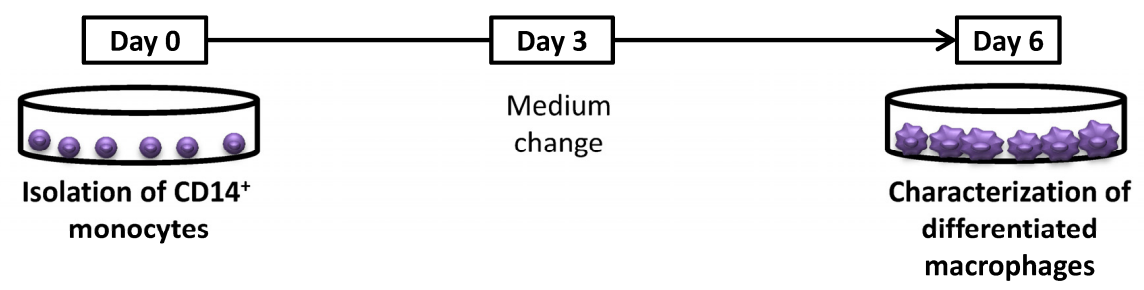

\section{Combinations of factors used during} macrophage differentiation:

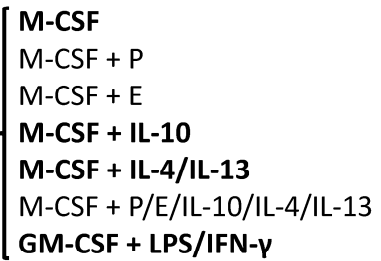

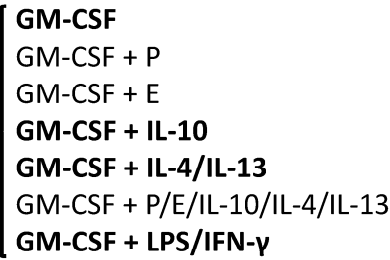

$\left\{\begin{array}{l}M-C S F \\ \text { GM-CSF } \\ M-C S F+G M-C S F \\ M-C S F+G M-C S F+I L-10 \\ \text { GM-CSF + LPS/IFN- } \gamma\end{array}\right.$

Figure 7. MACS-sorted CD14 ${ }^{+}$blood monocytes from healthy female volunteers were differentiated into macrophages in the presence of different stimuli for six days. All factors were added the first day (day 0), except for LPS and IFN- $\gamma$ that were added after three days (day 3). Blood monocytes (day 0) and differentiated macrophages (day 6) were characterized for cell surface marker expression, cytokine and chemokine production and gene expression. All combinations of factors were used in paper I, while those used in paper II are shown in bold. P: progesterone, E: estradiol.

Thus, in order to avoid the potential bias associated with using one growth factor, we chose to analyze the effects of potentially polarizing factors in macrophages differentiated under the influence of both M-CSF and GM-CSF (Fig. 7). One exception was the polarization of pro-inflammatory macrophages (classically activated or M1) that were used for comparison purposes. These macrophages were always stimulated with GM-CSF in combination with LPS and IFN- $\gamma$. In some experiments macrophages were differentiated with a combination of M-CSF and GMCSF.

In paper III, GM-CSF was used as a basic growth factor when analyzing the effects of placental factors on the polarization of macrophages, because placental conditioned medium alone failed to promote macrophage survival and differentiation. Since GM- 
CSF induces M1-like characteristics, this design made it possible to analyze the potential of placental-derived factors to promote an M2 phenotype in M1-primed macrophages, an effect that would have been difficult to observe in M-CSF-driven macrophages. A disadvantage of using GM-CSF is that it may not represent in vivo conditions where GM-CSF is likely to be produced at low levels in the absence of inflammatory stimuli (Hamilton, 2008). Thus, the relatively high levels of GM-CSF in our in vitro cultures could counteract the full potential of the placenta to induce regulatory properties at the steady state.

The most common experimental design for generating polarized macrophages is the differentiation in the presence of M-CSF or GM-CSF and the subsequent activation with cytokines (or TLR ligands) (Martinez et al., 2006; Jaguin et al., 2013; Kittan et $a l ., 2013)$. However, in papers I and II, macrophages were cultured in the presence of macrophage growth factors as well as polarizing factors (cytokines or hormones) from the first day of culture (Fig. 7). The aim of the experiments was to mimic the differentiation and polarization of decidual macrophages in vivo, and we reasoned that as soon as monocytes exit the blood circulation and enter the tissue they would be exposed to potentially polarizing factors produced at the fetal-maternal interface. The one exception was the classically activated macrophages that were stimulated with LPS and IFN- $\gamma$ after three days of differentiation with GM-CSF.

\section{Chemotaxis assay}

In paper II, a Transwell system was used to analyze the ability of macrophages to recruit different cell populations. Due to the difficulty in obtaining enough numbers of decidual macrophages from first trimester pregnancies, we used macrophages generated in vitro with M-CSF and IL-10 as a model for decidual macrophages. These macrophages displayed a chemokine profile similar to that of decidual macrophages as determined by gene expression (microarray) and protein (ELISA and multiplexed bead assay) analysis and should therefore behave similarly in the chemotaxis assay (discussed in more detail later on). The Transwell system consisted of two chambers 


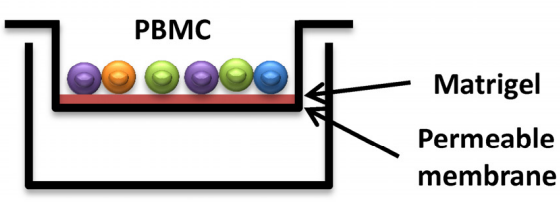

Medium

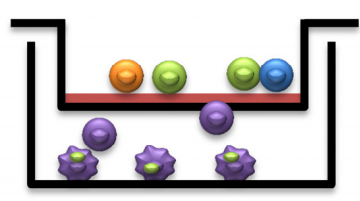

CFSE-labeled MФ

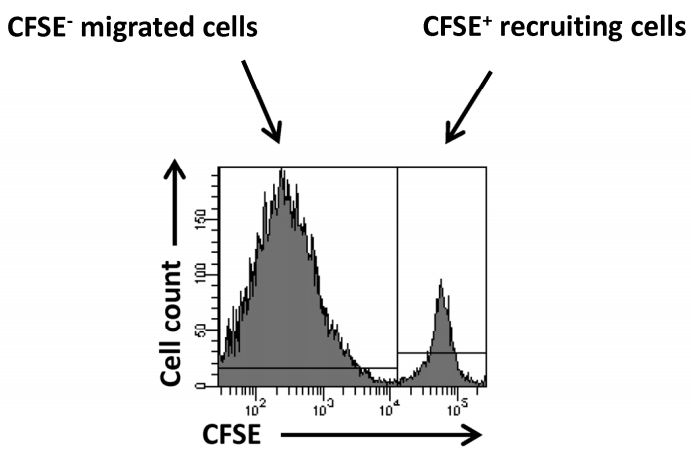

Figure 8. Schematic representation of the Transwell system used for the chemotaxis assay. Recruiting macrophages were CFSE-labeled and placed at the bottom of the Transwell system. Target cells (PBMC) were placed in a Matrigel-coated insert with semipermeable membrane. Wells with medium alone were used as control. PBMC were allowed to migrate overnight and were then characterized by flow cytometry.

separated by a Matrigel-coated semi-permeable membrane (Fig. 8). Matrigel is a biological mixture of basement membrane proteins and is commonly used as a substitute for extracellular matrix (Kleinman and Martin, 2005), and has for instance been used to study the migratory ability of invading trophoblast cells (Tarrade et al., 2001). As compared to the semi-permeable membrane alone, it provides a more physiological barrier, which cells must actively migrate through, reducing the spontaneous migration of cells. PBMC were placed in the upper chamber and were allowed to migrate through the membrane into the lower chamber containing macrophages. The recruiting macrophages were pre-labeled with the cell divisiontracking dye carboxyfluorescein diacetate succinimidyl ester (CFSE) to distinguish them from the recruited cells. The recruited cells were then counted by using 
TruCount tubes and were analyzed by flow cytometry to define the proportion of different cell populations.

Since we wanted to study the ability of macrophages to recruit different cell populations in an unbiased manner, we used PBMC as target cells. Similar systems have been used where the migratory ability of different cells was analyzed by placing PBMC in the upper chamber and by characterizing the migrated cells by flow cytometry (Hayashida et al., 2001; Huang et al., 2008). Although this approach provides a more in vivo-like system where different cell populations compete for chemokine ligands, it may impede the analysis of small populations such as Treg cells. Since we were interested in the Treg cell recruitment potential of macrophages, an option would have been to analyze the migratory ability of isolated Treg cells (or at least $\mathrm{CD}^{+} \mathrm{T}$ cells), which is perhaps the most commonly used approach in chemotaxis assays (Nguyen et al., 2009; Chenivesse et al., 2012). The use of macrophages in the lower chamber may have also interfered with the analysis of recruited cells (in particular small populations) and an alternative would have been to use conditioned media from macrophages. Although conditioned medium is commonly used (Wu et al., 2005; Huang et al., 2008), we chose to use macrophages because they could provide with the continuous production of chemotactic factors thus creating a chemokine gradient sustained over time.

\section{Th cell polarization; unstimulated versus stimulated cells}

In paper III, we studied the effects of placental explant conditioned medium (PE CM) on Th cell polarization and activation as well as the effect on Treg cell induction. In order to mimic the decidual microenvironment at the resting state, $\mathrm{CD} 4{ }^{+} \mathrm{T}$ cells were cultured with PE CM without TCR stimulation (termed unstimulated), and to test the ability of placental-derived factors to prevent or suppress Th cell activation, Th cells were stimulated by anti-CD3/CD28 antibodies to obtain TCR and co-stimulatory signals (Fig. 9). 


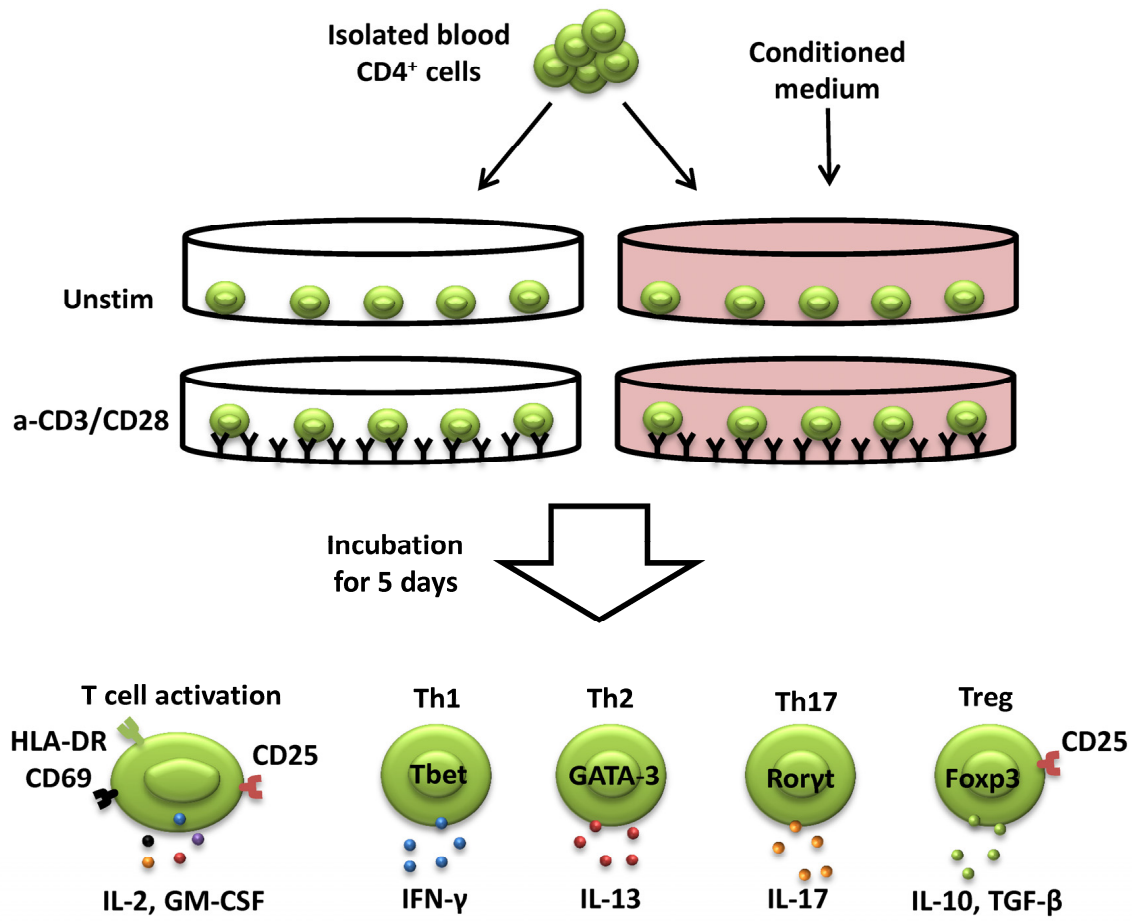

Figure 9. Design of the experiments used to study the effect of placental conditioned medium on Th cell activation and polarization and the induction of Treg cells. Isolated $\mathrm{CD}^{+} \mathrm{T}$ cells were cultured in uncoated or anti-CD3/CD28 Ab-coated wells and in the absence or presence of conditioned medium for five days. The cells were then characterized by flow cytometry, multiplexed bead assay, ELISA and/or real-time RT-PCR.

One obvious drawback of anti-CD3/CD28-stimulation is the strong and widespread activation of Th cells that does not mirror antigen-specific $\mathrm{T}$ cell activation in vivo (Tan et al., 2014). However, in order to study suppressive effects in vitro it is usually necessary to activate a large number of cells, and this is not easily achieved by antigen-stimulation. A strong stimulation was also necessary to induce a clear upregulation of activation-associated parameters and to be able to evaluate the potential suppressive function of placental factors within a reasonable number of experiments. The general activation of Th cells, including activation of Th1, Th2 and 
Th17 cells, also allowed evaluating the effects on Th cell activation regardless of the type of stimuli.

Since Treg cell induction may require TCR stimulation (Chen et al., 2003), the potential effect of PE CM on Treg cell induction could also be studied in antiCD3/CD28-stimulated Th cells in parallel with the effects on unstimulated Th cells. Although it is unclear to what extent Treg cells expand in response to fetal antigens in human pregnancy (Guerin et al., 2009; Ernerudh et al., 2011), this allowed us to evaluate the in vitro potential of placental factors to expand the Treg cell population by a model reflecting both antigen-specific and antigen-non-specific mechanisms.

\section{Treg cell suppression assay}

In paper III, we wanted to test whether the Treg cells induced by placental factors were functional. $\mathrm{CD}^{+} \mathrm{T}$ cells were cultured with PE CM for five days and the expanded population of Treg cells was isolated by flow cytometry-activated cell sorting (FACS) (Fig. 10). Since it is not possible to isolate viable cells based on the expression of Foxp3, Treg cells were defined as $\mathrm{CD} 4{ }^{\mathrm{dim}} \mathrm{CD} 25^{\text {high }}$ as previously described (Mjosberg et al., 2009). $\mathrm{CD}^{+} \mathrm{CD} 25^{-}$responder cells were isolated in parallel and were labeled with the cell division-tracking dye CFSE (Lyons, 2000). CFSE is a fluorescent dye that is incorporated into the cytosol of cells, and dilution of CFSE caused by proliferation of cells is then measured with flow cytometry. The responder cells were then stimulated with anti-CD3/CD28 antibodies and were cocultured with the placental-induced Treg cells for five more days at the ratio 2:1. As discussed above, the stimulation with anti-CD3/CD28 antibodies results in the robust activation of $\mathrm{T}$ cells that may not be reflective of in vivo activation. An alternative could have been to analyze the ability of Treg cells to downregulate the expression of the activation markers HLA-DR, CD69 and CD154 (CD40 ligand), which are more rapidly induced and may require a lower degree of stimulation (Canavan et al., 2012). Nevertheless, inhibition of proliferation assessed by CFSE is well-established and is one of the most common methods to define Treg cell function in vitro. 


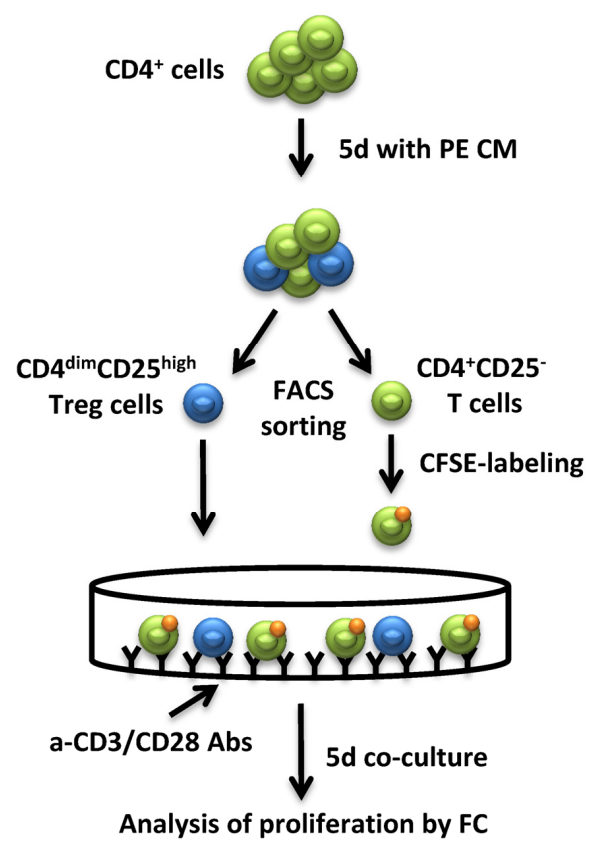

Figure 10. Treg cell suppression assay. Isolated $\mathrm{CD}^{+} \mathrm{T}$ cells were cultured in the presence of placental explant conditioned medium (PE CM) and after five days of culture, $\mathrm{CD} 4^{+} \mathrm{CD} 25^{-}$ responder cells and $\mathrm{CD} 4{ }^{\mathrm{dim}} \mathrm{CD} 25^{\text {high }}$ Treg cells were isolated by FACS. The responder cells were labeled with the cell division-tracking dye CFSE, stimulated with anti-CD3/CD28 antibodies (Abs) and co-cultured with the PE CM-induced Treg cells for five days at the ratio $2: 1$. Proliferation was assessed by flow cytometry (FC).

\section{First trimester placental explants}

In paper III, we aimed to study the ability of placental-derived factors to influence Th cell polarization and activation and to promote the polarization of Treg cells and homeostatic macrophages. First trimester placental villi were dissected into small pieces (termed placental explants) and were cultured overnight in culture medium. The conditioned medium was then used to stimulate macrophages and Th cells during their differentiation in vitro (Fig. 11). Although we hypothesized that trophoblast cells play a central role in immune modulation, the placenta harbors many other cell types, including mesenchymal stromal cells, endothelial cells and fetal macrophages that could potentially contribute to the production of soluble factors, as well as influence the function of trophoblast cells. Thus, one advantage of using placental explants is that it represents a physiological environment where trophoblast cells are cultured together with the cells that they are typically in contact with (Miller et al., 2005). The simple and rapid procedure to prepare placental explants also reduces cell damage and 
the risk of inducing stress-induced inflammation (Gohner et al., 2014). A

disadvantage is the inability to identify specific cell populations responsible for a certain property or mechanism. However, we used immunohistochemistry (IHC) to identify the cellular source of placental-derived factors that were identified as central for the induction of homeostatic leukocytes. We also analyzed the effect of isolated trophoblast cells in parallel with placental explants (discussed below).

\begin{tabular}{|c|c|c|c|}
\hline $\begin{array}{c}\text { Placental tissue } \\
\text { incubated overnight }\end{array}$ & $\begin{array}{l}\text { Conditioned } \\
\text { medium (CM) }\end{array}$ & $\begin{array}{l}\text { Polarization of Th } \\
\text { cells and } M \Phi \text { with } \mathrm{CM}\end{array}$ & $\begin{array}{l}\text { Characterization of } \\
\text { Th cells and MФ }\end{array}$ \\
\hline
\end{tabular}

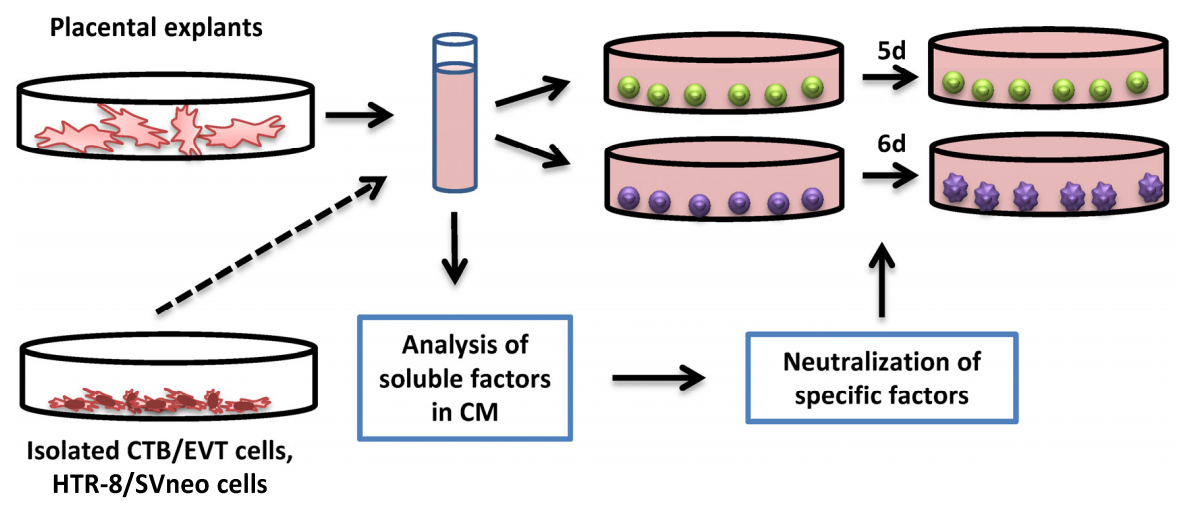

Figure 11. Placental tissue explants or isolated CTB and EVT cells from first trimester pregnancies, or the trophoblast cell line HTR-8/SVneo were cultured overnight in culture medium. The conditioned medium (CM) was used to stimulate Th cells or macrophages during their differentiation process. The $\mathrm{CM}$ was analyzed for cytokines, chemokines and growth factors, and some of these factors were blocked by neutralizing antibodies during the stimulation of Th cells or macrophages. CTB cells: Cytotrophoblast cells, EVT cells: Extravillous trophoblast cells, MФ: Macrophages. 


\section{First trimester primary trophoblast cells and the HTR-8/SVneo trophoblast cell line}

In paper III, we also tested if isolated trophoblast cells, the primary cell component of the placenta, could take part in the induction of homeostatic macrophages and Treg cells (Fig. 11). CTB cells, located under the superficial syncytiotrophoblast layer, represent a high proportion of all trophoblast cells contributing with great production of cytokines and growth factors. EVT cells are less frequent but due to their invasive nature they come into close contact with the maternal immune cells in the decidua and may have a great influence on the polarization of these cells. CTB and EVT cells were isolated by enzymatic digestion of placental villi followed by density centrifugation in a Percoll gradient. The purity of isolated EVT and CTB cells was routinely tested and was confirmed to be $>97 \%$ by immunostaining for cytokeratin 7 (all trophoblast cells) and HLA-G (EVT) as previously described (Lash et al., 2010). In addition, we compared primary trophoblast cells with the first trimester trophoblast cell line HTR8/SVneo, which shows many similarities with invasive EVT cells (Graham et al., 1993) and is commonly used as a surrogate for freshly isolated trophoblast cells.

Some obvious advantages in using HTR-8/SVneo cells are the unlimited availability and the reproducibility of experiments. However, like many other cell lines, HTR8/SVneo cells differ from primary trophoblast cells in many aspects (King et al., 2000c; Apps et al., 2009; Morales-Prieto et al., 2012) and data obtained from these cells may not accurately reflect the properties of trophoblast cells. On the other hand, the use of freshly isolated trophoblast cells is also associated with downsides. The prolonged separation procedure, for instance in comparison to placental explants, may cause cell damage and the induction of pro-inflammatory mediators. The high yield and purity of isolated trophoblast cells, or any other cells from solid tissues, require disaggregation of the tissue, which is commonly achieved by enzymatic digestion, a method also used by us. The specific effects of this treatment on the viability and function of trophoblast cells has not been thoroughly evaluated. However, detailed analysis of immune cells after mechanical or different enzymatic disaggregation procedures shows that a wide range of cell surface receptors is altered by the 
enzymatic treatment (Grange et al., 2011; Quatromoni et al., 2015). On the other hand, one advantage of enzymatic separation is that it may be associated with increased cell viability as compared to mechanical separation (Quatromoni et al., 2015). Although mechanical disaggregation of placental tissue may not be sufficient for the isolation of trophoblast cells (in contrast to isolation of immune cells), the use of a milder enzyme treatment has been proposed to improve the quality of isolated first trimester trophoblast cells (Stenqvist et al., 2008) and should be considered in future experiments.

Taken together, a combination of several approaches (the use of placental explants, IHC analysis of placental tissue, isolated primary trophoblast cells and the HTR8/SVneo cell line), should limit the impact of individual disadvantages on the results.

\section{Microarray data}

In papers I and II, microarray analysis was used to compare the gene expression profiles of decidual macrophages and in vitro macrophage populations. The different macrophage subsets analyzed in the respective papers are shown in Table V. Because of the large number of stimulation combinations, the in vitro macrophages were differentiated at different occasions. Macrophages that were generated in parallel, i.e. from blood monocytes from the same donor, were grouped together to form one "experiment set" as shown in Table V. As a consequence, blood monocytes and for instance GM(LPS/IFN $\gamma$ ) macrophages include more samples than for instance GM(IL-10) macrophages that were only included in one experiment set. However, to avoid bias caused by uneven number of replicates between groups of samples, only samples within the same experiment set were compared statistically (see below).

Microarray technology has become widely used and is a powerful method to analyze the expression of a large number of genes in an unbiased manner. This is a major advantage in comparison with other methods, where the number of markers or analytes is rather limited (for instance flow cytometry and multiplexed bead assay). 
Table V. Samples used in the microarray analysis ${ }^{*}$

\begin{tabular}{|c|c|c|c|}
\hline Exp. set & Monocyte/macrophage subset & Paper I & Paper II \\
\hline \multirow{2}{*}{$\mathbf{A}$} & Blood monocytes (pregnant) & $\mathrm{x}$ & $\mathrm{x}$ \\
\hline & Decidual macrophages & $\mathrm{x}$ & $\mathrm{x}$ \\
\hline \multirow{8}{*}{ B } & Blood monocytes (non-pregnant) & $\mathrm{x}$ & $\mathrm{x}$ \\
\hline & M & $\mathrm{x}$ & $\mathrm{x}$ \\
\hline & $\mathrm{M}+\mathrm{IL}-10$ & $\mathrm{x}$ & $\mathrm{x}$ \\
\hline & $\mathrm{M}+\mathrm{IL}-4$ + IL-13 & $\mathrm{x}$ & $\mathrm{x}$ \\
\hline & $\mathrm{M}+\mathrm{P}$ & $\mathrm{x}$ & \\
\hline & $M+E$ & $\mathrm{x}$ & \\
\hline & $\mathrm{M}+\mathrm{IL}-10+\mathrm{IL}-4+\mathrm{IL}-13+\mathrm{P}+\mathrm{E}$ & $\mathrm{x}$ & \\
\hline & $\mathrm{GM}+\mathrm{LPS}+\mathrm{IFN}-\gamma$ & $\mathrm{x}$ & $\mathrm{x}$ \\
\hline \multirow{8}{*}{$\mathbf{C}$} & Blood monocytes (non-pregnant) & $\mathrm{x}$ & $\mathrm{x}$ \\
\hline & GM & $\mathrm{x}$ & $\mathrm{x}$ \\
\hline & GM + IL-10 & $\mathrm{x}$ & $\mathrm{x}$ \\
\hline & GM + IL-4 + IL-13 & $\mathrm{x}$ & $\mathrm{x}$ \\
\hline & $\mathrm{GM}+\mathrm{P}$ & $\mathrm{x}$ & \\
\hline & $\mathrm{GM}+\mathrm{E}$ & $\mathrm{x}$ & \\
\hline & GM + IL-10 + IL-4 + IL-13 + P + E & $\mathrm{x}$ & \\
\hline & $\mathrm{GM}+\mathrm{LPS}+\mathrm{IFN}-\gamma$ & $\mathrm{x}$ & $\mathrm{x}$ \\
\hline \multirow{6}{*}{ D } & Blood monocytes (non-pregnant) & $\mathrm{x}$ & \\
\hline & M & $\mathrm{x}$ & \\
\hline & GM & $\mathrm{x}$ & \\
\hline & $\mathrm{GM}+\mathrm{M}$ & $\mathrm{x}$ & \\
\hline & $\mathrm{GM}+\mathrm{M}+\mathrm{IL}-10$ & $\mathrm{x}$ & \\
\hline & $\mathrm{GM}+\mathrm{LPS}+\mathrm{IFN}-\gamma$ & $\mathrm{x}$ & \\
\hline
\end{tabular}

"Sample details are available at the GEO database http://www.ncbi.nlm.nih.gov/projects/geo/ under Acc. GSE30595

M: M-CSF, GM: GM-CSF, P: progesterone, E: estradiol, Exp. set: Experiment set

The possibility of comparing the different macrophage subsets based on gene expression profiles in papers I and II was particularly valuable considering the phenotypic overlap and lack of unique markers for different macrophage subpopulations.

Regarding statistical analysis of the data, the high number of genes analyzed in combination with few replicates for each condition presents a statistical challenge. Although the risk of mass significance is reduced by using false discovery rate (FDR) 
correction, the opposite risk exists, the risk of not finding biologically relevant differences. However, in contrast to global gene expression analysis that includes tens of thousands of genes, we chose to focus on 100 decidual macrophage-specific genes in paper I and 589 genes associated with immune function in paper II (described in more detail in the papers). A total of 420 macrophage-related genes were analyzed in paper I; however, only the 100 mentioned above were subject to statistical analysis. Further, the statistical comparison was not performed between all groups of samples. Instead, differentiated macrophages were compared with their matching blood monocytes, thus limiting the number of comparisons. Importantly, the interpretation of the results was not with focus on statistical differences, but rather on fold change patterns and results from hierarchical clustering, which in an unbiased manner grouped samples with similar gene expression patterns together.

\section{Statistical methods}

Details regarding the statistical methods used are provided in each paper. All analyses were performed by using Graph Pad Prism version 5.0 or 6.0, with the exception of microarray data, which were analyzed with GeneSpring GX 11.0.2 or 12.6. In general, flow cytometry and real-time RT-PCR data were analyzed with parametrical tests, because the majority of data was normally distributed. In contrast, cytokine and chemokine data from multiplex bead assays and ELISA were analyzed with nonparametrical tests. $p$ values $\leq 0.05$ were considered statistically significant $(* p \leq 0.05$, $* * \mathrm{p} \leq 0.01, * * * \mathrm{p} \leq 0.001)$.

For microarray data, statistical differences were calculated using ANOVA and the Tukey post-hoc test with Benjamini-Hochberg FDR multiple testing correction. Genes were defined as differentially regulated when $p \leq 0.05$ and the mean fold change regulation was at least $+/-2$. 


\section{Results and Discussion}

\section{Phenotype and subpopulations of decidual macrophages}

In paper I, the phenotype of CD14 ${ }^{+}$decidual macrophages from first trimester pregnancies was analyzed by flow cytometry, and $\mathrm{CD} 14^{+}$blood monocytes were analyzed as a reference population. The M2 markers CD163, CD206 and CD209 and the potential new marker NRP-1 were expected to be upregulated on decidual macrophages. In contrast, intercellular adhesion molecule (ICAM)-3 was used as a control for being downregulated, since this was the case at the gene expression level in decidual macrophages as compared to blood monocytes (Gustafsson et al., 2008).

An early observation was that decidual macrophages not only showed lower expression of ICAM-3 as compared to blood monocytes (Fig. 12A), but could also be further divided into two distinct populations based on the level of ICAM-3 expression (Fig. 12B). ICAM-3 ${ }^{\text {low }}$ and ICAM-3 ${ }^{\text {high }}$ decidual macrophages showed differential expression of all markers analyzed, with ICAM- $3^{\text {low }}$ cells displaying significantly higher expression of CD163, CD206, CD209 and NRP-1 than ICAM-3 ${ }^{\text {high }}$ cells (Fig. 12C). Since ICAM-3 ${ }^{\text {high }}$ macrophages were phenotypically more closely related to blood monocytes, we speculated that the ICAM- $3^{\text {high }}$ population might represent newly recruited monocytes or a contamination of blood in the sample. During the preparation of paper I, CD14 ${ }^{+}$decidual macrophages were also reported to subdivide into two populations based on CD11c expression $\left(\mathrm{CD} 11 \mathrm{c}^{\text {low }}\right.$ or $\left.\mathrm{CD} 11 \mathrm{c}^{\text {high }}\right)$, with similar differential expression of CD206 and CD209 (Houser et al., 2011). When analyzing decidual macrophages with both markers we found a good correlation between ICAM-3 and CD11c expression, and CD209 and CD206 showed similar patterns of expression on ICAM- $3^{\text {low }}$ and ICAM-3 ${ }^{\text {high }}$, as on CD $11 c^{\text {low }}$ and CD $11 c^{\text {high }}$ decidual macrophages, respectively (paper I, Fig. 1D-E). 
A.

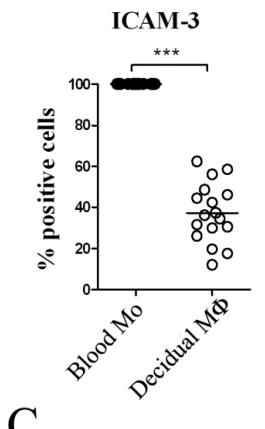

CD163 (SR)

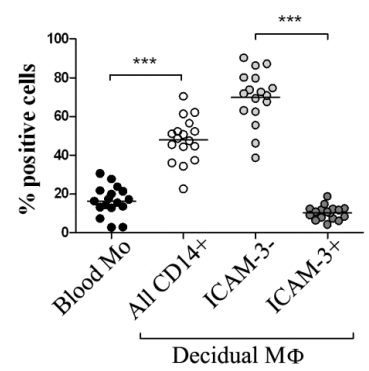

CD209 (DC-SIGN)

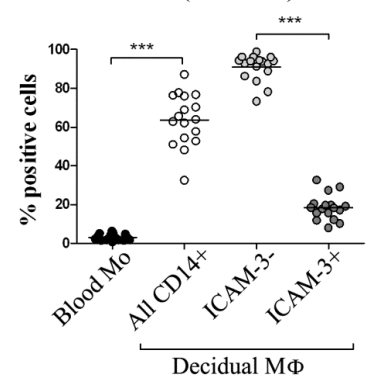

B.

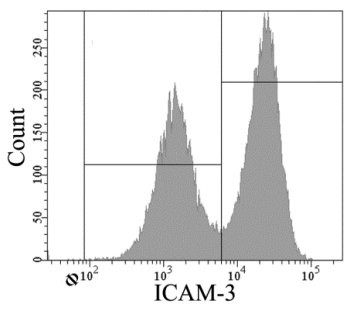

CD206 (MR)

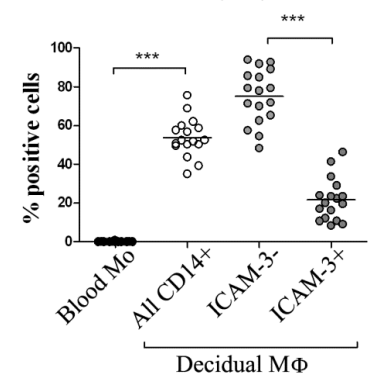

NRP-1 (neuropilin-1)

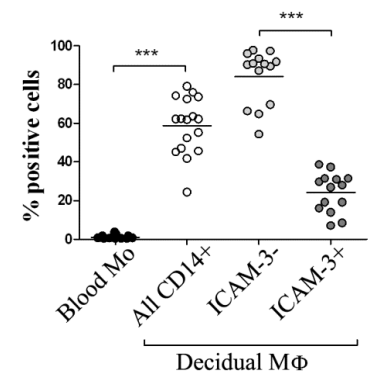

Figure 12. Phenotypic characterization of decidual macrophages (MФ). Flow cytometry was used to characterize the phenotype of $\mathrm{CD} 14^{+}$blood monocytes and $\mathrm{CD} 14^{+}$decidual MФ from first trimester healthy pregnant women $(n=17)$. A, Expression of ICAM-3 in percent of $\mathrm{CD} 14^{+}$blood monocytes and $\mathrm{CD} 14^{+}$decidual MФ. B, The histogram shows that $\mathrm{CD} 14^{+}$ decidual MФ can be divided into two distinct subpopulations based on ICAM-3 fluorescence intensity: CD $14^{+} \mathrm{ICAM}_{-} 3^{- \text {(low) }}$ (left peak) and CD $14^{+} \mathrm{ICAM}^{-3^{+}}{ }^{\text {(high) }}$ (right peak). C, Expression of cell surface markers in percent of $\mathrm{CD} 14^{+}$blood monocytes or decidual $\mathrm{M} \Phi$ shown as the total $\mathrm{CD} 14^{+}$population or as the CD14 ${ }^{+}$ICAM- $3^{-}$or $\mathrm{CD} 14^{+} \mathrm{ICAM}-3^{+}$ subpopulations. All bars represent means. ${ }^{* * *} \mathrm{p} \leq 0.001$. 
Although the CD11 $\mathrm{c}^{\text {high }}$ population was proposed to be a unique subpopulation of tissue macrophages (Houser et al., 2011), several observations suggest that the ICAM$3^{\text {high }} \mathrm{CD} 11 \mathrm{c}^{\text {high }}$ population consists of immature macrophages and blood monocytes.

First, by analyzing the relative expression of ICAM-3 (expressed as the mean fluorescence intensity (MFI) ratio) we observed a dramatic downregulation of ICAM3 in all in vitro macrophage subsets, regardless of stimuli (Fig. 13). This observation is supported by a study reporting the rapid downregulation of ICAM-3 upon macrophage differentiation, but also in response to transendothelial migration in an in vitro model (Estecha et al., 2012). The downregulation of ICAM-3 mRNA occurred within $24 \mathrm{~h}$ and was maintained through the differentiation process. Second, by using lineage-tracing techniques, Ly6 $\mathrm{C}^{\text {hi }}$ monocytes were demonstrated to give rise to intestinal tissue macrophages expressing CD163 and CD206 (Bain et al., 2013). These characteristics were absent on newly arrived monocytes and gradually increased upon macrophage maturation. Analogous populations were found in the human intestine, where $\mathrm{CD} 14^{+}$cells expressing high levels of $\mathrm{CD} 11 \mathrm{c}$ corresponded with an immature $\mathrm{CD} 163^{\text {low }} \mathrm{CD} 209^{\text {low }}$ phenotype while $\mathrm{CD} 11 \mathrm{c}^{\text {low }}$ cells corresponded with a mature
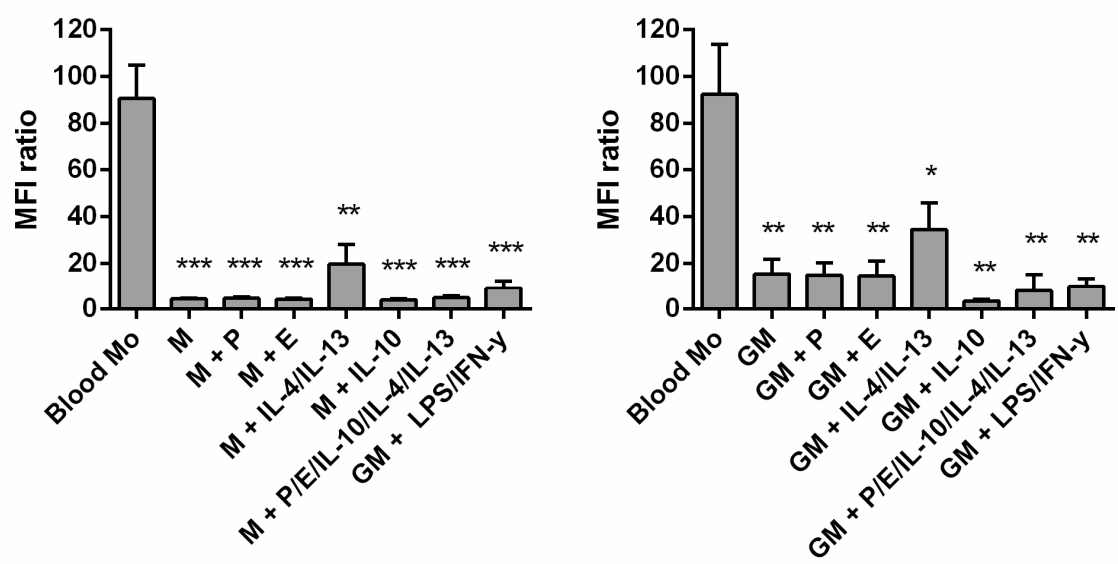

Figure 13. Relative expression of ICAM-3 on blood monocytes and different in vitro differentiated macrophages. Expression of ICAM-3 is expressed as the mean fluorescence intensity (MFI) ratio, which was calculated as the ratio between the MFI of specific Abstained cells and the MFI of isotype control-stained cells. ${ }^{*} \mathrm{p} \leq 0.05, * * \mathrm{p} \leq 0.01, * * * \mathrm{p} \leq$ 0.001 (versus blood monocytes). E: estradiol, GM: GM-CSF, M: M-CSF, Mo: monocytes, P: progesterone. 
CD163 ${ }^{\text {high }} \mathrm{CD} 209^{\text {high }}$ tissue macrophage phenotype. A similar pattern of continuous Ly6C $\mathrm{C}^{\text {hi }}$ monocyte recruitment to the uterus in mice (Tagliani et al., 2011), supports the presence of macrophages at different stages of differentiation, including newly arrived $\mathrm{CD} 11 \mathrm{c}^{\text {high }}$ monocytes. In addition, Tagliani et al. used a novel technique to characterize intra- and extravascular leukocytes, and found that intravascular monocytes constituted a high proportion of uterine decidual monocytes/macrophages (Tagliani et al., 2011). When analyzing decidual mononuclear cells by flow cytometry, the presence of decidual intravascular monocytes is inevitable. Given that blood monocytes express strikingly higher levels of ICAM-3 than differentiated macrophages, the ICAM- $3^{\text {high }}$ population should largely consist of intravascular monocytes and newly recruited monocytes. Thus, although both ICAM-3 and CD11c are potentially useful markers for identifying a more homogenous population of fully differentiated decidual macrophages, this issue deserves further investigation. With this in mind, results presented below, apart from flow cytometry data, represent the total $\mathrm{CD} 14^{+}$decidual macrophage population.

Taken together, $\mathrm{CD} 14^{+}$decidual macrophages showed a distinct pattern of expression with the majority of cells being $\mathrm{CD} 163^{+} \mathrm{CD} 206^{+} \mathrm{CD} 209^{+}$and NRP- $1^{+}$, a pattern that was more pronounced on the ICAM- ${ }^{\text {low }}$ population. These markers were therefore used to evaluate the similarity between decidual macrophages and the different in vitro polarized macrophages.

\section{The influence of M-CSF and IL-10 on decidual macrophage polarization}

Increasing evidence suggests that decidual macrophages predominantly show an immune suppressive phenotype, but the factors that induce these characteristics have not been characterized. The view of pregnancy as a Th2-dominated condition in combination with the decidual macrophage expression of CD206 and CD209 often described in Th2-associated macrophages (Stein et al., 1992; Soilleux et al., 2002; Kammerer et al., 2003; Puig-Kroger et al., 2004; Laskarin et al., 2005), suggested that 
IL-4 and IL-13 may influence decidual macrophage polarization. However, a great number of additional factors, like other cytokines, growth factors and hormones, have been proposed to induce macrophages with M2 properties, and could potentially be involved in the differentiation and polarization of decidual macrophages.

In paper I, we therefore evaluated the influence of M-CSF, GM-CSF, IL-4 and IL-13, IL-10, progesterone and estradiol on macrophage differentiation and polarization by generating macrophages in vitro in the presence of these factors. Our results showed that M-CSF and in particular IL-10 (in combination with both GM-CSF and M-CSF) could induce macrophages with characteristics of decidual macrophages $\left(\mathrm{CD} 163^{+}\right.$ CD $206^{+} \mathrm{CD}^{209^{+}} \mathrm{NRP}^{+}{ }^{+}$ICAM- $^{\text {low }}$ ) (Fig. 14). In contrast, IL-4 and IL-13 induced a different pattern of expression, while progesterone and estradiol had no influence on macrophage polarization (Fig. 14). M-CSF/IL-10-stimulated and decidual macrophages also showed comparable cytokine secretion patterns, based on analysis of IL-6, IL-10, TNF and CCL4 (paper I, Fig. 6B). In addition, this resemblance was largely supported in paper II where the IL-12 family cytokines IL-12, IL-23, IL-27 and IL-35 and the chemokine profiles of the different macrophages were analyzed (paper II, Fig. 1B-E and Fig. 4-5 and 6A).

The close relationship between decidual macrophages and macrophages polarized in vitro under the influence of M-CSF and IL-10 was supported by gene expression analysis by using hierarchical clustering, which in an unbiased manner grouped samples with similar gene expression patterns together (Fig. 15, paper I, Fig. 4A and paper II, Fig. 1A and 6A). Similar clustering patterns were observed regardless of the functional groups of genes analyzed (immune modulating-, tissue remodeling-, cell cycle- or cell metabolism/transport-associated), indicating that decidual and MCSF/IL-10-driven macrophages are not only related in terms of immune function, but also in terms of more general biological processes (Fig. 15). These findings reflect the involvement of different macrophage subsets in various biological aspects, for instance metabolism and angiogenesis, which has been increasingly acknowledged (Pollard, 2009; Biswas and Mantovani, 2012). 
CD14

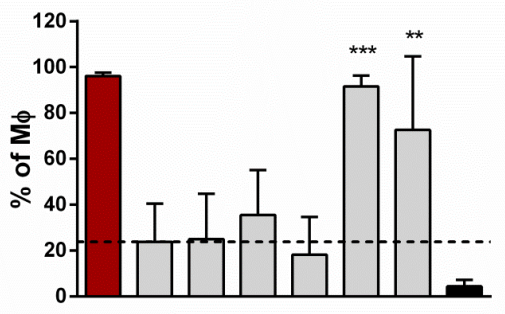

ICAM-3

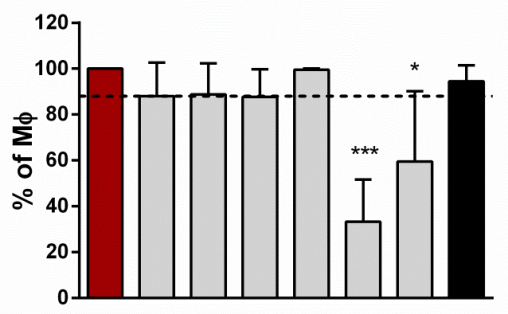

CD163

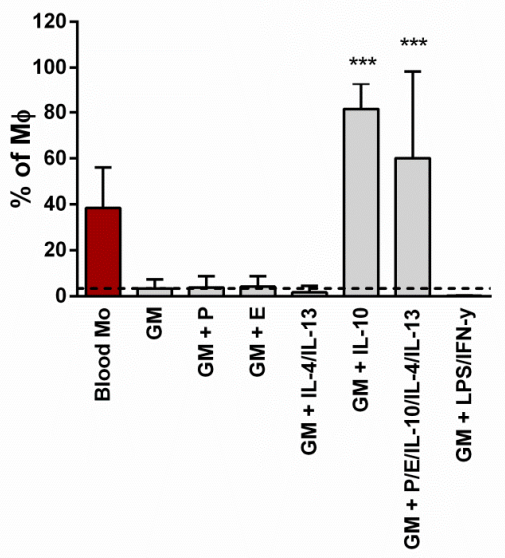

CD14

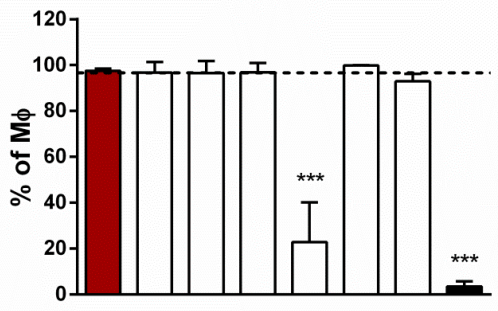

ICAM-3

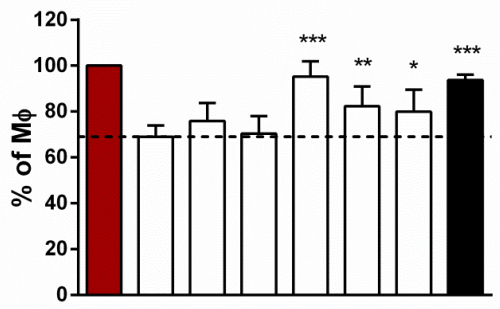

CD163

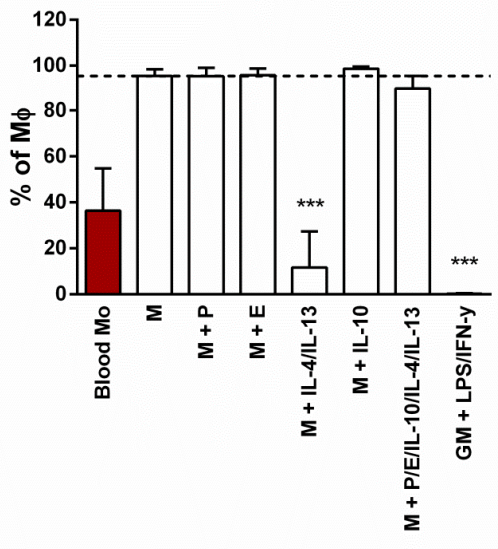

Figure 14. Effect of different stimuli on macrophage (MФ) polarization. CD14 $4^{+}$blood monocytes isolated from non-pregnant healthy women were cultured for six days and phenotypically characterized by flow cytometry. Left panels show macrophages cultured with GM-CSF (GM) and right panels with M-CSF (M) in addition to other stimuli. Data is presented as the percentage of cells expressing a specific marker. Mean + SD from five individual experiments, ${ }^{*} \mathrm{p} \leq 0.05, * * \mathrm{p} \leq 0.01,{ }^{* * *} \mathrm{p} \leq 0.001$ (versus $\mathrm{GM}$ or $\mathrm{M}$ macrophages, of which the means are highlighted by the dotted lines). E: estradiol, P: progesterone, Mo: monocytes 

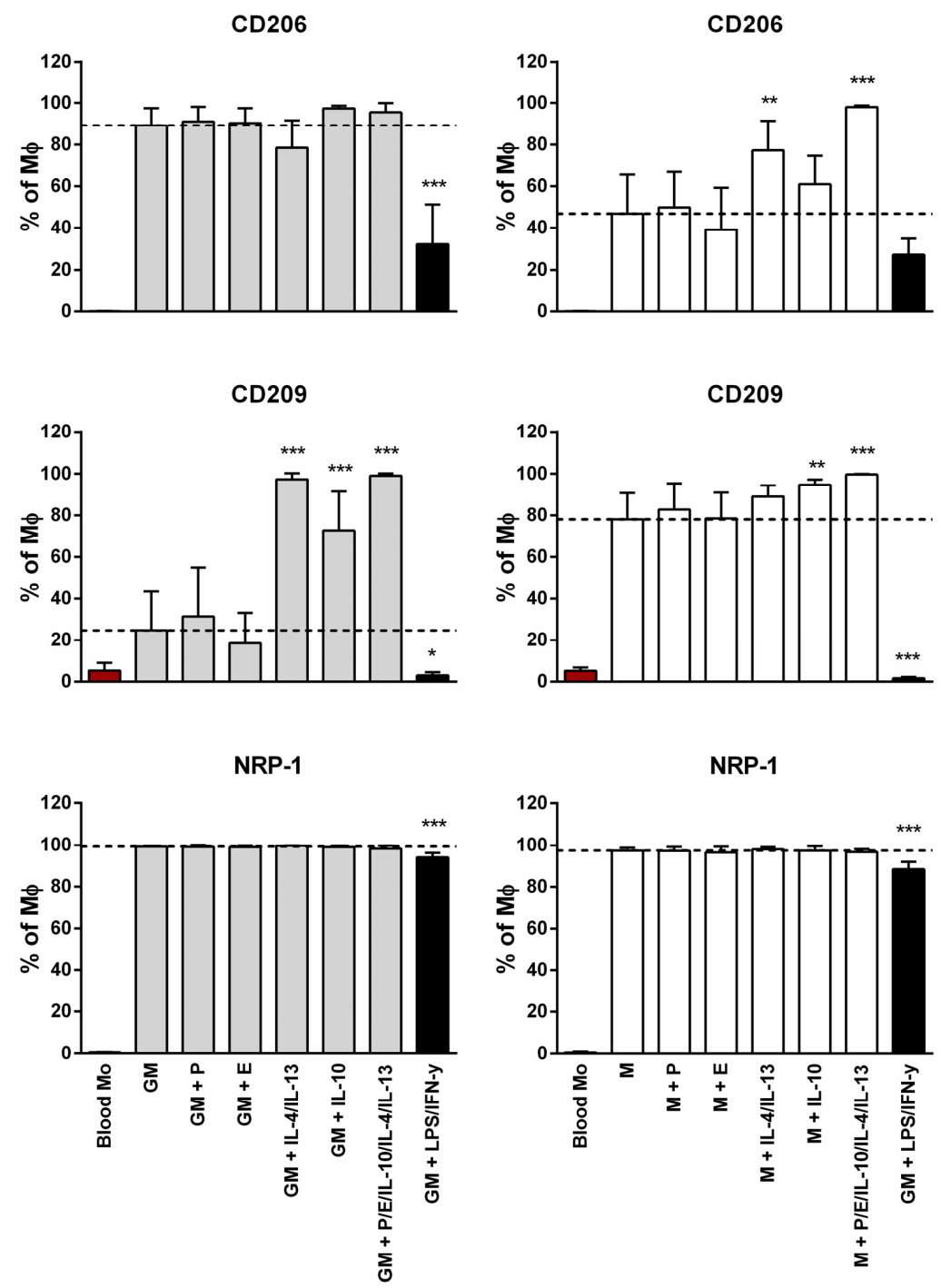

Figure 14. Cont. 
A. Immune modulating

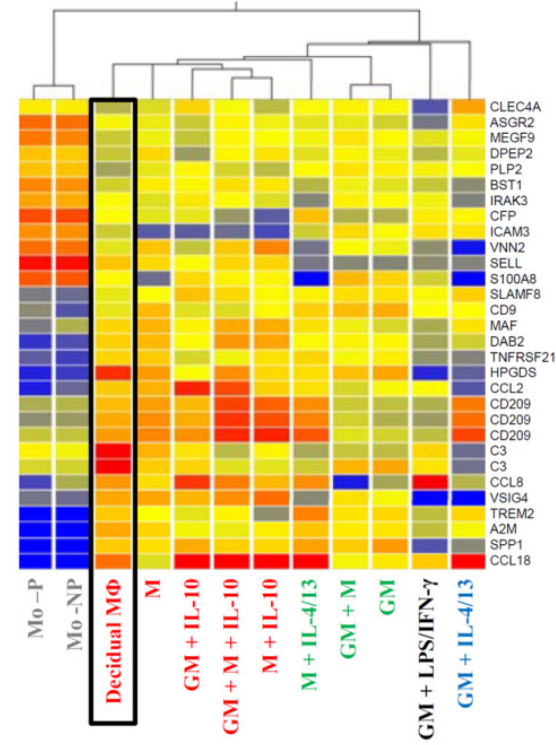

B. Tissue remodeling

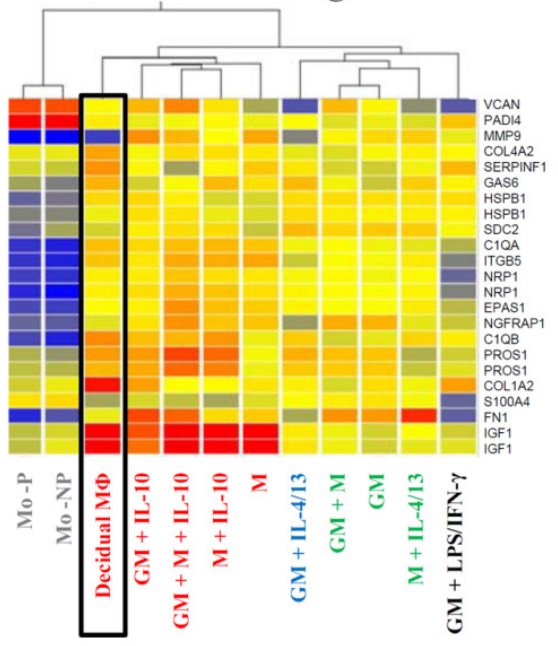

C. Cell cycle-related

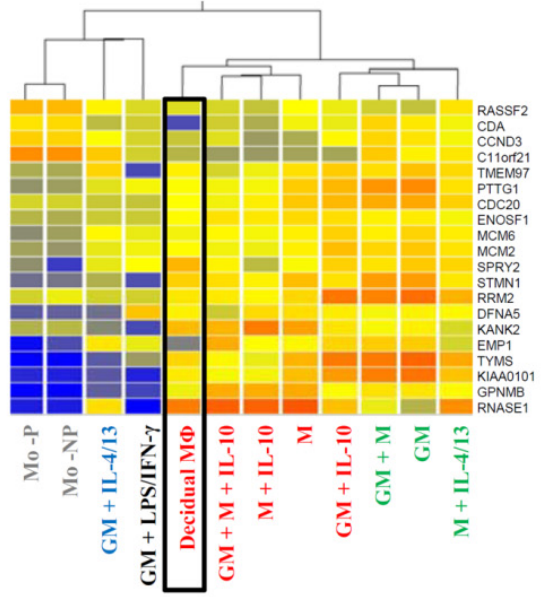

D. Cell metabolism/transport

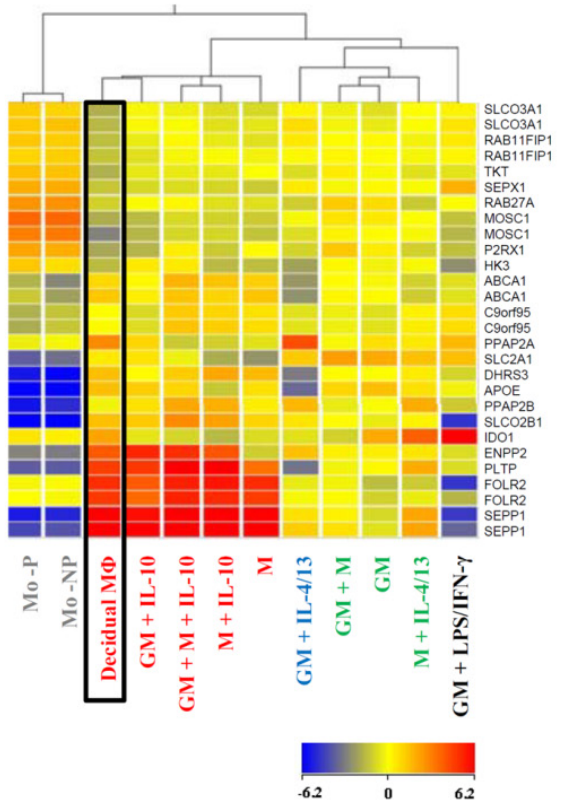


Figure 15. Hierarchical clustering of conditions after grouping genes according to functions: A, Immune modulating, B, Tissue remodeling, C, Cell cycle-related and D, Cell metabolism/transport. Conditions with similar expression profiles were grouped together to form a clustering tree. Each column represents the averaged values of at least three samples of each condition (only two for GM+M+IL-10). The color map represents the normalized intensity values (normalized to the median of all samples) of genes (rows), where red and blue represents up- and downregulated genes, respectively. Some genes are represented by more than one probe and appear therefore more than once in the heat maps. Mo -P: Monocytes from pregnant women, Mo -NP: Monocytes from non-pregnant women, M: MCSF, GM: GM-CSF. MФ: macrophages.

Despite the obvious differences between the complex decidual environment and our in vitro model, decidual and M(IL-10) macrophages were surprisingly similar, supporting an important role of M-CSF and IL-10 in the regulation of decidual macrophages. In paper III, we also showed that macrophages cultured in the presence of conditioned medium from first trimester placental tissue acquire characteristics of decidual macrophages $\left(\mathrm{CD} 14^{+} \mathrm{CD} 163^{+} \mathrm{CD} 206^{+} \mathrm{CD} 209^{+}\right.$and $\mathrm{IL}-10^{+} \mathrm{CCL} 18^{+} \mathrm{IL}^{-12} 2^{-} \mathrm{IL}-$ $23^{-}$) (paper III, Fig. 1). Furthermore, by using blocking antibodies, we showed that MCSF and IL-10 were partly responsible for this phenotype (paper III, Fig. 6A and B). Of note, in this model, the effects of M-CSF and IL-10 were apparent at physiological levels and as part of the complete pool of placental-derived factors. In addition, the decidual macrophage characteristics were induced despite the presence of GM-CSF (which was used a basic growth factor), which in paper I was shown to counteract the M2-polarizing effect of M-CSF.

Thus in papers I-III, we show that M-CSF and IL-10 are potent inducers of the characteristics of decidual macrophages, and likely play a major role in the regulation of macrophages at the fetal-maternal interface. M-CSF has been proposed to promote macrophages with an increased threshold for activation, which may be important for maintaining tissue integrity (Hamilton, 2008). Similarly, IL-10 is a homeostatic cytokine constitutively produced both at the steady state and during inflammation to limit the extent of the immune response (Mosser and Zhang, 2008). During pregnancy, the ability of M-CSF and IL-10 to reduce the inflammatory potential of decidual macrophages may be crucial for preserving a homeostatic fetal environment. 
Further, GM-CSF was observed to counteract the effect of M-CSF, and macrophages stimulated under the influence of both GM-CSF and M-CSF showed characteristics of GM-CSF macrophages (including cell surface expression, cytokine production and gene expression). The antagonistic effect of GM-CSF is likely to be dose-dependent (Hamilton, 2008; Brocheriou et al., 2011) and thus at low levels, local production of GM-CSF (for instance by the placenta as shown in paper III, Fig. 5A and Table I) is likely compatible with the induction of homeostatic macrophages in vivo. However, increased GM-CSF levels in relation to M-CSF could disturb the differentiation of homeostatic decidual macrophages contributing to the pathogenesis of pregnancy complications. Since our data also showed that IL-10 could restore the homeostatic M2 phenotype in macrophages cultured with GM-CSF, it could be argued that GMCSF together with IL-10 could regulate the differentiation of decidual macrophages. However, the numbers of uterine macrophages are not altered in GM-CSF-deficient mice (Moldenhauer et al., 2010), which contrasts data from M-CSF-deficient mice where uterine macrophages are almost absent (Wiktor-Jedrzejczak and Gordon, 1996).

The relevance of M-CSF and IL-10 in pregnancy is supported by their increased levels at the fetal-maternal interface (Bartocci et al., 1986; Pampfer et al., 1992; Hanna et al., 2000), and by observations of increased rates of spontaneous abortions and increased susceptibility to infection-induced fetal loss in both M-CSF- (Pollard et al., 1991; Qiu et al., 2009) and IL-10-deficient mice (Chaouat et al., 1995; Robertson et al., 2007; Thaxton et al., 2009). Thus, we suggest that M-CSF and IL-10 promote homeostatic M2 macrophages that are able to protect the fetus against invading pathogens without compromising fetal survival. In addition, increased decidual GMCSF levels (Huang et al., 2010), and reduced decidual and placental IL-10 have been described in preeclamptic patients (Hennessy et al., 1999; Schonkeren et al., 2011). Further, IL-10-induced M2 macrophages have been shown to promote, and M1-like macrophages to inhibit, trophoblast invasion in vitro (Renaud et al., 2005; Renaud et $a l .$, 2007). The poor placentation associated with preeclampsia may therefore in part be attributed to failure of decidual macrophages to acquire tissue remodeling properties that are associated with the M2 polarized phenotype. 
Our results also showed that Th2 cytokines induced macrophages with characteristics distinct from M-CSF-, IL-10-, and decidual macrophages. Notably, upon culture with a cocktail of cytokines and hormones that included IL-4, IL-13 and IL-10 (Fig. 14), macrophages showed characteristics most similar to IL-10-stimulated macrophages. These results suggest that in the presence of IL-10, Th2 cytokines have little influence on the polarization of decidual macrophages. Although this is in contrast to the initially proposed Th2 paradigm in pregnancy (Wegmann et al., 1993), these results are supported by the preferential accumulation of Treg cells, but not Th2 cells, in the first trimester human decidua (Mjosberg et al., 2010). Taken together, our results are consistent with a predominantly homeostatic and tolerogenic, rather than a Th2dominated, environment at the fetal-maternal interface.

\section{Decidual macrophage function}

\section{Cytokine production by decidual macrophages}

Although decidual macrophages have mainly been attributed immune suppressive or homeostatic properties, it has been pointed out that they differ from both M1 and M2 macrophages (Houser et al., 2011). This interpretation was partly based on the comparison of the transcriptional profile of decidual macrophages $\left(\mathrm{CD} 14^{+} \mathrm{CD} 11 \mathrm{c}^{\text {high }}\right.$ and $\mathrm{CD} 14^{+} \mathrm{CD} 11 \mathrm{c}^{\text {low }}$ ) with the gene profiles of M1 and M2 macrophages previously reported by Martinez and colleagues (Martinez et al., 2006). In that study, M1 and M2 macrophages were generated in vitro under the influence of M-CSF, LPS and IFN- $\gamma$ or M-CSF and IL-4, representing the typical Th1-associated and Th2-associated macrophages, respectively. Thus the lack of correlation between decidual macrophages and M2 macrophages was based on Th2-associated macrophages and not M2 macrophages in a broad sense, for instance those induced by IL-10, M-CSF or glucocorticoids (Mantovani et al., 2004). In this regard, our data showing that IL-4 and IL-13 induce macrophages with characteristics clearly distinct from decidual macrophages, is in line with the data by Houser et al. (2011). However, decidual macrophages were also observed to secrete pro-inflammatory cytokines, including 
TNF and IL-1 $\beta$ (in addition to the production of for instance IL-10 and TGF- $\beta$ ), suggesting that they also possess inflammatory potential (Houser et al., 2011).

In paper I, we analyzed the spontaneous production of IL-10, IL-1 $\beta$, IL-6 and TNF by decidual macrophages and by the different in vitro macrophage subsets. Our data showed that $\mathrm{CD} 14^{+}$decidual macrophages indeed produce IL-6 and TNF (Fig. 16).
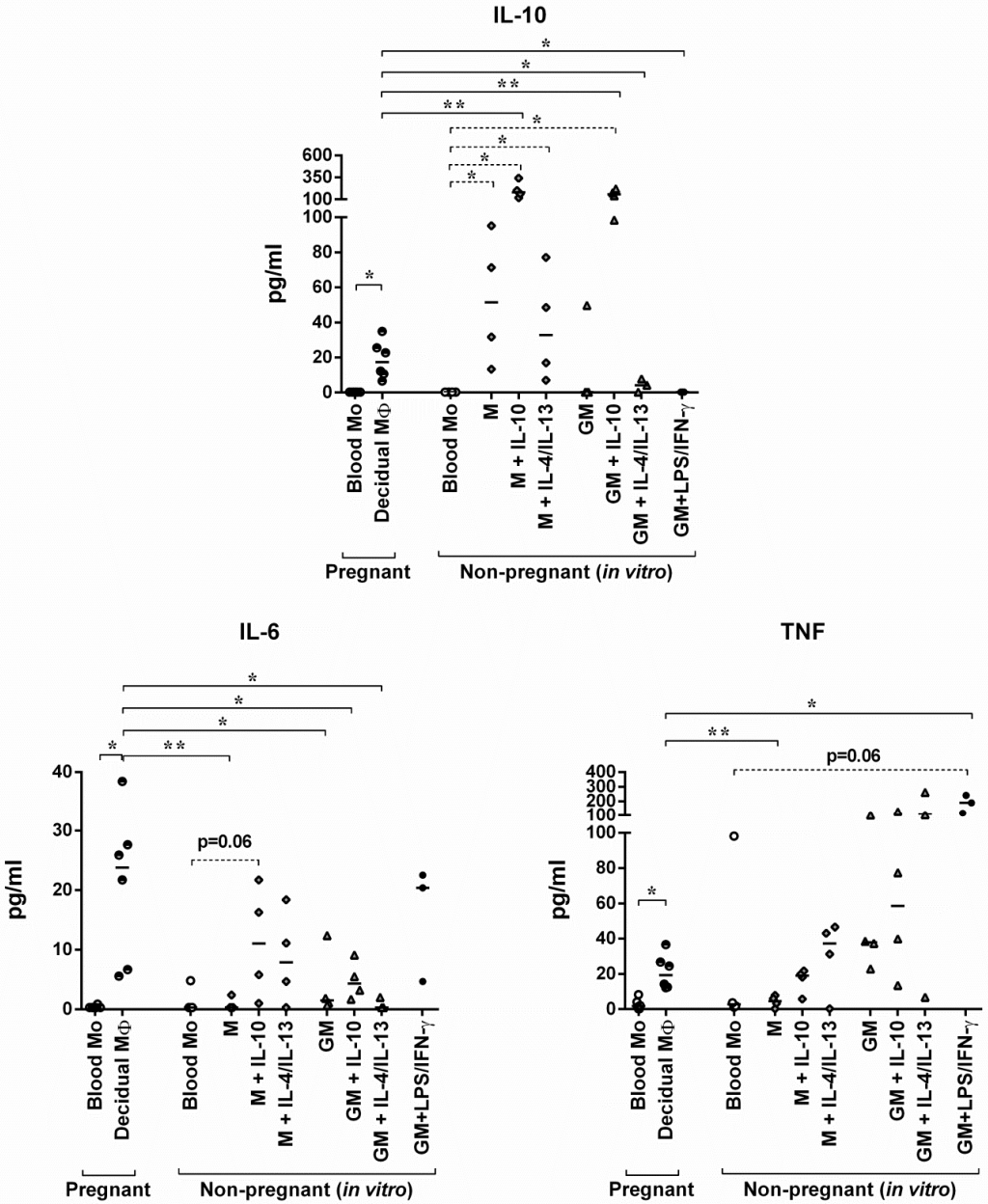

Figure 16. Cytokine secretion pattern in decidual macrophages and different in vitro $\mathrm{M} 1$ and M2 macrophage populations $(n=3-6)$. Monocytes and macrophages were isolated and cultured overnight, after which cytokine levels were analyzed with a Multiplex bead assay. All bars represent medians. ${ }^{*} \mathrm{p} \leq 0.05,{ }^{* *} \mathrm{p} \leq 0.01$. МФ: macrophages. 
However, these cytokines were produced at similar levels by macrophages with immune suppressive phenotypes, for instance those polarized in the presence of $\mathrm{M}$ CSF and IL-10. In addition, TNF was produced at significantly higher levels by proinflammatory GM(LPS/IFN- $\gamma$ ) macrophages than by decidual macrophages. These results demonstrate that production of pro-inflammatory cytokines by itself not necessarily correlates with pro-inflammatory macrophage activity, and the effect of specific cytokines is likely context-dependent. In fact, IL-6 has been shown to upregulate CD163 (Buechler et al., 2000) and to potentiate the M2 phenotype of tumor-associated macrophages by enhancing CD206 and CD209 expression (Roca et al., 2009; Dominguez-Soto et al., 2011). Thus, secretion of moderate levels of inflammatory cytokines does not contradict the classification of decidual macrophages as M2-polarized with potentially suppressive function.

Further, a certain level of pro-inflammatory activity seems to be compatible, if not necessary, with homeostatic or suppressive functions. For instance, in a study showing that term decidual stromal cells could induce CD $25^{\text {high }}$ Foxp $3^{+}$Treg cells and mediate suppression of allo-responses, neutralization of IFN- $\gamma$ reduced the suppressive function of decidual stromal cells (Erkers et al., 2013). In this study, it was also shown that administration of high levels of IFN- $\gamma$ inhibited this suppressive potential, while low IFN- $\gamma$ levels had no effect. The ability of IFN- $\gamma$ to enhance suppressive function was also observed in co-cultures of decidual NK cells and macrophages, which together induced functionally suppressive Foxp $3^{+} \mathrm{CD} 25^{\text {high }}$ Treg cells; in this model IFN- $\gamma$ blockade partially reduced the Treg cell induction (Vacca et al., 2010).

In paper II, we extended the panel of cytokines by analyzing the IL-12 family cytokines IL-12, IL-23, IL-27 and IL-35, which are influential in tailoring adaptive immune responses (Goriely and Goldman, 2008; Vignali and Kuchroo, 2012). These cytokines are unique among cytokines in that they are heterodimeric and composed of subunits that are shared between the members of the family. IL-12 is formed by p35 and p40, while p40 together with p19 instead forms IL-23. p35 also pairs with Ebi3 to form IL-35, whereas Ebi3 together with p28 forms IL-27. Despite having common subunits, these cytokines have divergent functions, ranging from Th1- and Th17- 
IL-12 (p70)

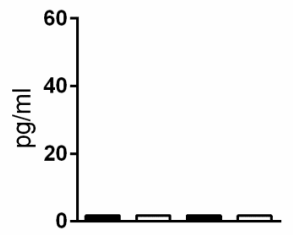

IL-27

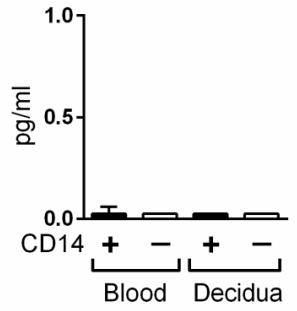

IL-23

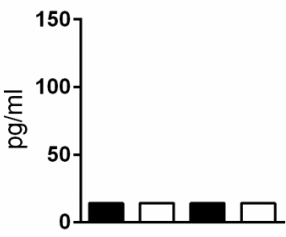

IL-35

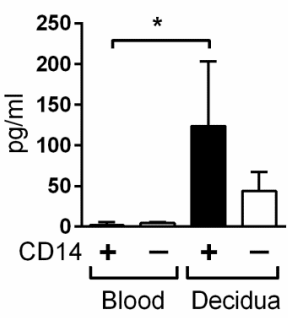

Figure 17. Secretion of IL-12 family cytokines by isolated CD14 ${ }^{+}(\mathrm{n}=4-10)$ and CD14depleted $(n=2-7)$ mononuclear cells from blood and decidua (from first trimester pregnancies). The concentration of IL-12 family cytokines was measured by multiplexed bead assay or ELISA; the graphs show the medians and range. ${ }^{*} \mathrm{p} \leq 0.05$.

promoting (IL-12 and IL-23) or both pro- and anti-inflammatory (depending on the context, IL-27) to immune suppressive and Treg cell-inducing (IL-35). While macrophages are a well-known source of IL-12, IL-23 and IL-27, production of IL-35 has to date mainly been associated with Treg cell function (Collison et al., 2007). Consistent with an M2 phenotype, CD14 ${ }^{+}$decidual macrophages (but also CD14depleted decidual mononuclear cells) from first trimester pregnancies failed to spontaneously produce IL-12, IL-23 and IL-27 (Fig. 17). Accordingly, they expressed low mRNA levels of one ( $p 28$ for IL-27) or both ( $p 35$ and $p 40$ for IL-12; $p 40$ and $p 19$ for IL-23) corresponding cytokine subunits (paper II, Fig. 1C). Among the in vitro macrophage subsets, only GM(LPS/IFN- $\gamma$ ) macrophages produced significant levels of IL-12, IL-23 and IL-27 (Fig. 18), supporting that these cytokines are mainly induced during inflammatory conditions (Vignali and Kuchroo, 2012). In contrast, IL35 was detected in conditioned medium from decidual mononuclear cells and was 
particularly produced by $\mathrm{CD} 14^{+}$decidual macrophages at significantly higher levels than by blood monocytes (Fig. 17). Accordingly, mRNA expression of Ebi3, which forms IL-35 together with p35, was expressed at significantly higher levels in decidual macrophages than in blood monocytes (paper II, Fig. 1C). Although $p 35$ mRNA was detected at low levels in decidual macrophages, GM(LPS/IFN- $\gamma$ ) macrophages that secreted both IL-12 and IL-35 (Fig. 18) (both share the p35 subunit) also expressed low p35 mRNA levels (paper II, Fig. 1E), indicating that failure to detect p35

IL-12 (p70)

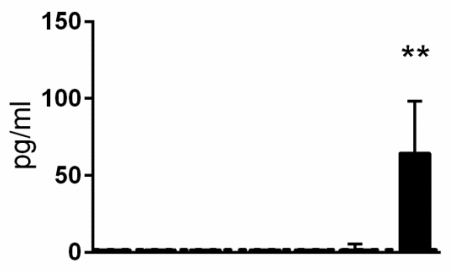

IL-27

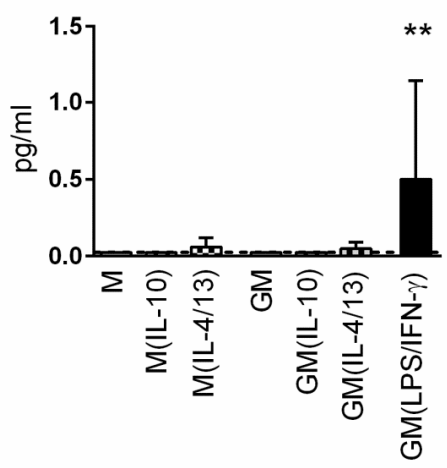

IL-23

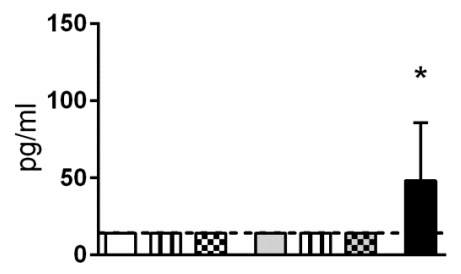

IL-35

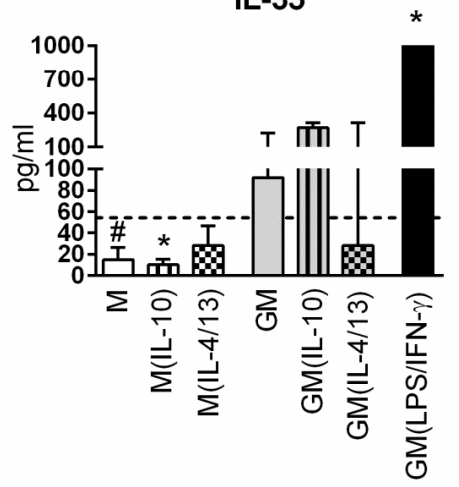

Figure 18. Secretion of IL-12 family cytokines by different in vitro macrophage subsets $(\mathrm{n}=4-5)$. In vitro macrophages were generated from $\mathrm{CD} 14^{+}$monocytes from non-pregnant women. The concentration of IL-12 family cytokines was measured by multiplexed bead assay or ELISA; the graphs show the medians and range. Dotted lines denote the median of blood monocytes. ${ }^{*} \mathrm{p} \leq 0.05,{ }^{* *} \mathrm{p} \leq 0.01$, \# $\mathrm{p}=0.06$ (versus $\mathrm{CD} 14^{+}$blood monocytes). M: MCSF, GM: GM-CSF. 
may be due to methodological issues, possibly kinetic-related, rather than lack of expression by these cells.

IL-35 has been shown to contribute to suppressive function and to protect against several autoimmune and inflammatory disorders and to alleviate graft-versus-host disease in allogeneic stem cell transplantation (Collison et al., 2007; Niedbala et al., 2007; Collison et al., 2010; Olson et al., 2012; Shen et al., 2014; Liu et al., 2015), making it an interesting candidate for promoting fetal tolerance. The production of IL35 by decidual macrophages is however intriguing, since IL-35 has been shown to be mainly restricted to Treg cells in mice (Collison et al., 2007) and, in addition, the production by human Treg cells has been debated (Allan et al., 2008; Bardel et al., 2008). However, human placental trophoblast cells (where IL-35 was first described) have been demonstrated to secrete IL-35 (Devergne et al., 1997; Mao et al., 2013), implying that in humans, IL-35 may have other cellular sources than Treg cells. Although decidual macrophage-derived IL-35 could promote conversion of Treg cells in the decidua, IL-35-induced Treg cells represent a unique population of IL-35producing $\mathrm{CD}^{+}{ }^{+}$Foxp3 $3^{-} \mathrm{IL}_{-10}{ }^{-} \mathrm{TGF}-\beta^{-}$Treg cells (Collison et al., 2010) that have not yet been characterized in human (or mouse) decidua.

A recent study showed that $\mathrm{CD} 163^{+} \mathrm{IL}-10^{+}$macrophages in the murine testis express the IL-35 subunits Ebi3 and p35, which were shown to limit lymphocyte infiltration and IFN- $\gamma$ production, suggesting a role for macrophage-derived IL-35 in the maintenance of immune privilege (Terayama et al., 2014). Contrary to our expectations, the pattern of IL-35 secretion by in vitro macrophages did not correlate with immune regulatory phenotype since pro-inflammatory GM(LPS/IFN- $\gamma$ ) macrophages produced the highest levels of IL-35 in parallel with IL-12, IL-23 and IL-27 (Fig. 18). However, this is in line with the suggested role of IL-35 as an inducible cytokine that suppresses on-going inflammatory responses, in contrast to "house-keeping" cytokines (like TGF- $\beta$ ) that rather may prevent the onset of inflammation (Li et al., 2012; Vignali and Kuchroo, 2012). In addition, Ebi3 alone may have immune modulatory potential. For instance, it has been shown to limit IL12 production, thereby inhibiting Th1 induction during LPS-challenge (Zheng et al., 
2013). A possible mechanism would be by competitive binding to $\mathrm{p} 35$, the shared subunit of IL-12 and IL-35 (Vignali and Kuchroo, 2012).

Whereas the cytokine profile of decidual macrophages supports a homeostatic role at the resting state, it is less clear to what extent they are involved in the protection against invading pathogens. Expression of pattern recognition receptors (CD163, CD206 and CD209) on decidual macrophages suggests that microbial stimuli could trigger an inflammatory immune response and the induction of pro-inflammatory mediators (Fabriek et al., 2009; Geijtenbeek and Gringhuis, 2009). Indeed, decidual macrophages have been shown to respond with increased production of TNF and IL$1 \beta$ in response to microbial stimuli (Heikkinen et al., 2003; Singh et al., 2005; Houser et al., 2011). A recent report also demonstrated that decidual macrophages express mRNA for TLR1-9, of which TLR2, TLR3, and TLR4 where confirmed at the protein level (Duriez et al., 2014). TLR-ligation resulted in the increased production of several pro-inflammatory cytokines and chemokines, including IL-1 $\beta$, TNF, IL-6, GM-CSF, CCL5 and CXCL8. Interestingly, the Th1 cell-recruiting chemokine CXCL10 was not induced and both IL-10 and IL-1RA (which antagonizes IL-1 $\beta$ ) were induced in parallel with the pro-inflammatory cytokines (with the exception of TLR9 activation) (Duriez et al., 2014). The observation that IL-10 was the cytokine most strongly induced and that IL-1RA was secreted at levels higher than IL-1 $\beta$ even upon stimulation, supports that decidual macrophages maintain a high threshold of activation under inflammatory conditions, a property that may be crucial for preventing inflammation-induced fetal loss.

Furthermore, multiple factors present in vivo may influence decidual macrophages by further down-modulating their pro-inflammatory potential. Potential factors include cytokines produced by the placenta, like M-CSF and IL-10 as discussed earlier, but also pregnancy-associated hormones. Although we did not observe any direct effects of physiological doses of progesterone and estradiol on macrophage polarization (Fig. 14 and paper I, supplemental Table I), these and other hormones may modulate the effects of inflammatory stimuli on decidual macrophages. In support of this notion, treatment with progesterone or the glucocorticoid receptor agonist dexamethasone 
reduced the production of nitric oxide, IL-12 and TNF from mouse macrophages in response to LPS (Miller and Hunt, 1998; Jones et al., 2008).

In summary, these data suggest that decidual macrophages are predominantly tolerogenic at the steady state and that the inflammatory potential may be under strict control to preserve a microenvironment compatible with fetal development.

\section{Influence of decidual macrophages on the polarization of Th1, Th2, Th17 and Treg cells}

The cytokine secretion pattern of decidual macrophages, as well as data from gene expression profiling and phenotypic characterization, indicate a predominantly immune suppressive function of these cells. This is supported by the observations that isolated decidual macrophages were more suppressive than blood monocytes in a mixed lymphocyte reaction (Mizuno et al., 1994), and that they did not spontaneously induce proliferation of allogenic T cells (Kammerer et al., 2003). However, a more recent study showed that co-culture of isolated decidual macrophages with allogeneic $\mathrm{CD}^{+} \mathrm{T}$ cells indeed resulted in the proliferation of $\mathrm{T}$ cells, and this was shown to be preferentially within the $\mathrm{CD}^{+}$population (Vacca et al., 2010). By studying the decidual macrophage-primed $\mathrm{CD}^{+} \mathrm{T}$ cells in more detail, Vacca et al showed that a major proportion of the $\mathrm{CD} 4^{+} \mathrm{T}$ cells consisted of cells with a Treg cell phenotype $\left(\mathrm{CD} 127^{\text {low }} \mathrm{CTLA}-4^{+}\right.$and $\left.\mathrm{CD} 39^{+}\right)$and with suppressive activity. Thus, analyzing the influence of decidual macrophages on specific Th cell populations should be more informative than studying proliferation of the entire $\mathrm{CD}^{+}$or $\mathrm{CD}^{+} \mathrm{T}$ cell population. Since the influence of decidual macrophages on specific Th cell subsets is unknown, we analyzed the ability of decidual macrophages to promote the expansion of Treg cells in parallel with the effects on Th1, Th2 and Th17 cell polarization (paper II). By using decidual macrophage conditioned medium during the polarization of $\mathrm{CD} 4^{+} \mathrm{T}$ cells for five days, we showed that decidual macrophages promote the selective expansion of Treg cells, defined as Foxp $3^{+}, \mathrm{CD} 4{ }^{\text {dim }} \mathrm{CD} 25^{\text {high }}$ or $\mathrm{CD} 25^{\text {high }} \mathrm{Foxp}^{+}$(Fig. 19A-C). In contrast, the proportions of $\mathrm{CD}^{+} \mathrm{T}$ cells expressing the lineage-specific transcription factors T-bet, GATA-3 or Ror $\gamma \mathrm{t}$ were not increased, showing that 

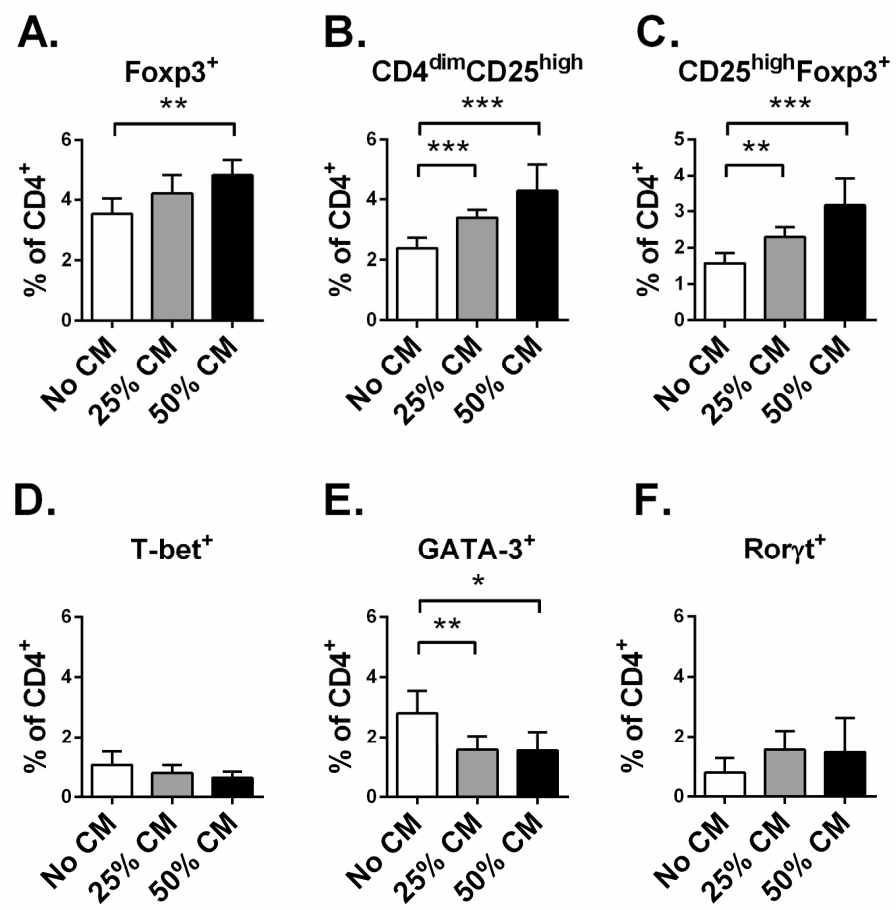

Figure 19. Decidual macrophages preferentially induce Treg cells. The graphs show the effect of $25 \%$ and $50 \%$ conditioned medium $(\mathrm{CM})$ from first trimester decidual macrophages on the polarization of Treg cells, defined as (A) Foxp3 $3^{+}$, (B) $\mathrm{CD} 4{ }^{\mathrm{dim}} \mathrm{CD} 25^{\text {high }}$ or (C) CD25 ${ }^{\text {high }}$ Foxp $^{+}$, and on (D) T-bet ${ }^{+}$Th1, (E) GATA- $3^{+}$Th2 and (F) Roryt ${ }^{+}$Th17 CD4 ${ }^{+} \mathrm{T}$ cells. All data was analyzed by flow cytometry; bars show the mean + SD from eight (No CM and $25 \% \mathrm{CM})$ or three $(50 \% \mathrm{CM})$ experiments. ${ }^{*} \mathrm{p} \leq 0.05,{ }^{* *} \mathrm{p} \leq 0.01,{ }^{* * *} \mathrm{p} \leq 0.001$.

decidual macrophages do not spontaneously induce Th1, Th2 or Th17 cell polarization (Fig. 19D-F). Our results differ with the results reported by Vacca et al, where decidual macrophages alone did not induce Treg cells, but required the interaction with decidual NK cells (Vacca et al., 2010). These discrepancies are likely due to differences in experimental design, for instance the use of $\mathrm{CD}^{+} \mathrm{T}$ cells (Vacca et al., 2010) in contrast to isolated $\mathrm{CD}^{+} \mathrm{T}$ cells and the use of isolated decidual macrophages (Vacca et al., 2010) in contrast to conditioned medium. It may be speculated that although cytokines secreted by decidual macrophages promote Treg cell expansion in vitro (as was the case in our experiments), signals provided by cell- 
contact could counteract this effect, which could explain the lack of Treg cell induction observed in decidual macrophage-T cell co-cultures (Vacca et al., 2010). However, a recent paper showed that isolated term $\mathrm{CD} 14^{+} \mathrm{CD} 209^{+}$decidual macrophages induced proliferation of Foxp $3^{+} \mathrm{T}$ cells and that the percentage of proliferating Foxp $3^{+} \mathrm{T}$ cells negatively correlated with the percentage of proliferating non-Treg cells (Foxp3-), which was interpreted as suppressive activity within the Foxp $3^{+}$Treg cell population (Hsu et al., 2012). This finding supports the ability of decidual macrophages to promote Treg cells also by cell-contact.

Several macrophage-derived factors may contribute to expand the Treg cell population, including the established Treg cell-inducing factors TGF- $\beta$ and IL-10 (Groux et al., 1996; Chen et al., 2003; Rao et al., 2005). In addition, the emerging role of CCL18 as a Treg cell-inducing agent (Chang et al., 2010) and the high production of CCL18 by decidual macrophages (Fig. 20 and Gustafsson et al., 2008) implicates this chemokine in the Treg cell induction process. Interestingly, CD $163^{+} \mathrm{CD} 206^{+} \mathrm{IL}-$ $10^{\text {high }}$ CCL $18^{\text {high }}$ macrophages induced by M-CSF from multipotent stromal cells (thus resembling decidual macrophages) were shown to induce $\mathrm{CD} 4^{+} \mathrm{CD} 25^{\text {high }} \mathrm{Foxp} 3^{+}$Treg cells by a CCL18-dependent mechanism (Melief et al., 2013). Thus, the role of CCL18 in promoting immune homeostasis at the fetal-maternal interface deserves further attention.

As mentioned, the ability of decidual macrophages to influence the polarization of Th1, Th2 or Th17 cells has, to our knowledge, not been reported. However, allogeneic T cells co-cultured with decidual macrophages produced much lower levels of Th1associated IFN- $\gamma$, as compared with T cells co-cultured with blood monocytes (Sayama et al., 2013). Although Th1 cells were not specifically analyzed, this study corroborates our findings by showing that decidual macrophages are poor inducers of Th1-associated immune responses.

Taken together, our findings support the view that decidual macrophages show predominantly homeostatic functions at the resting state by promoting the preferential expansion of $\mathrm{CD} 25^{\text {high }} \mathrm{Foxp}^{+}$Treg cells, which is in line with the accumulation of Treg cells in the human decidua and their importance for maintained immune 
homeostasis during pregnancy (Tilburgs et al., 2006; Mjosberg et al., 2010). As discussed for cytokines, the ability of decidual macrophages to influence Th cell responses during inflammation is of major interest and has implications for pregnancy complications. Although cytokine data suggest that the pro-inflammatory potential of decidual macrophages is somewhat limited, functional studies will be necessary to support this idea.

\section{A potential role for decidual macrophages in controlling cell trafficking at the fetal-maternal interface}

Chemokines play an essential role in the trafficking of leukocytes during inflammation but they also contribute to homeostasis by promoting tissue-specific homing (Griffith et al., 2014). Although chemokines have gained much interest in several aspects of reproductive immunology (Du et al., 2014b), the role of decidual macrophages in contributing to the chemokine environment and the positioning of cells within the decidua has been surprisingly overlooked. NK cells have been attributed the ability to direct trophoblast invasion through production of the chemokines CXCL10 and CXCL8 and the interaction with their receptors CXCR3 and CXCR1 on trophoblast cells (Hanna et al., 2006). In paper II, by comparing the $\mathrm{CD}_{1} 4^{+}$and CD14-depleted decidual mononuclear cell populations, we show that chemokine production is mainly restricted to decidual macrophages (paper II, Fig. 3), including production of CXCL10. Although we did not analyze CXCL8, other studies comparing decidual $\mathrm{CD} 14^{+}$macrophages and $\mathrm{CD} 56^{+} \mathrm{NK}$ cells support that decidual macrophages also constitute the predominant source of CXCL8 (Li et al., 2009; Pitman et al., 2013). Further, decidual stromal cells or trophoblast cells are also a potential source of chemokines. Decidual stromal cells have been proposed to regulate monocyte recruitment by CCL2 (Lockwood et al., 2006) and, in mice, to control (or rather limit) the recruitment of $\mathrm{T}$ cells to the decidua by their inability to upregulate CXCR3 ligands (CXCL9 and CXCL10) and CCL5 (Nancy et al., 2012). Also the recruitment of decidual NK cells has been proposed to be controlled by decidual stromal cell production of CXCR3 ligands (CXCL10 and CXCL11) (Lockwood et al., 
2013). Trophoblast cells have been proposed to control both NK cell and monocyte recruitment to the uterus (Drake et al., 2001; Hanna et al., 2003; Abrahams et al., 2005).

Challenging these observations, our unpublished data show that within the decidua, $\mathrm{CD}^{+} 0^{+}$stromal cells in general secrete lower levels of chemokines than the CD10non-stromal compartment that mainly comprises immune cells (data to be published). Second, chemokine levels in conditioned media from decidual tissues were in general higher than in conditioned media from placental tissues. These data are based on the protein secretion analysis of ten different chemokines including CCL2, CXCL10, CCL22 and CCL18 (data to be published). Since decidual macrophages were a major chemokine source among decidual leukocytes it is tempting to speculate that decidual macrophages have a major influence in the control of leukocyte (and trophoblast) cell trafficking at the fetal-maternal interface and deserves to be further investigated.

To facilitate interpretation of the results, we analyzed the chemokine profile of decidual macrophages in relation to the different in vitro macrophage subsets. By this, we showed that decidual macrophages preferentially secrete the monocyte- and Treg cell-associated chemokines CCL2 and CCL18. In contrast, the Th1-, Th2- and Th17recruiting chemokines CXCL10, CCL22 and CCL20 were produced at low levels from decidual macrophages, both in relation to the production of CCL2 and CCL18 and in relation to the production from other macrophage subsets (Fig. 20). Similar to phenotypic and cytokine analyses, M(IL-10) macrophages showed a chemokine profile similar to decidual macrophages, while both M or GM(IL-4/IL-13) and

Figure 20. Chemokine profile of decidual macrophages and different in vitro macrophage subsets. Decidual macrophages and blood monocytes $(n=6$; for CCL20, $n=10)$ were isolated from first trimester human pregnancies. In vitro macrophages were generated from CD $14^{+}$ monocytes from non-pregnant women $(n=3-4)$. Cells were cultured overnight and the concentration of chemokines in the conditioned media was analyzed by multiplex bead assay or ELISA (medians are shown). Dotted horizontal lines denote the median of blood monocytes from pregnant women (for decidual macrophages) or from non-pregnant women (for in vitro macrophages). ${ }^{*} \mathrm{p} \leq 0.05,{ }^{* *} \mathrm{p} \leq 0.01, \# \mathrm{p}=0.06$ (versus blood monocytes). Dec MФ: decidual macrophages, M: M-CSF, GM: GM-CSF. 
A.

CXCL1-3

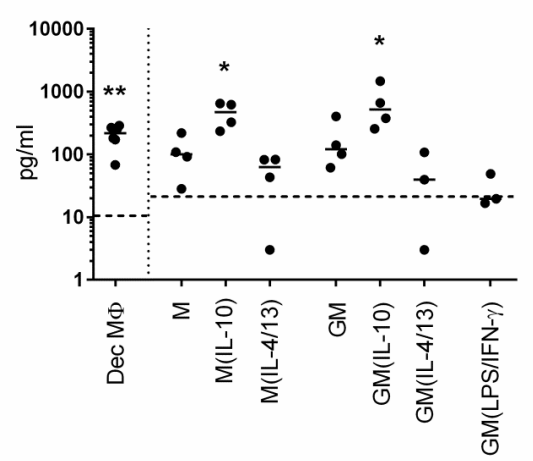

c.

CXCL10

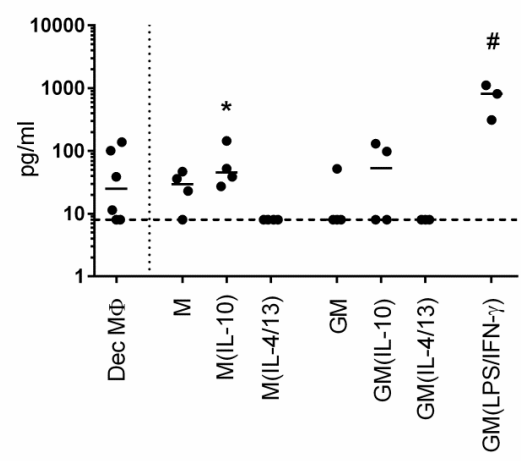

E.

CCL20

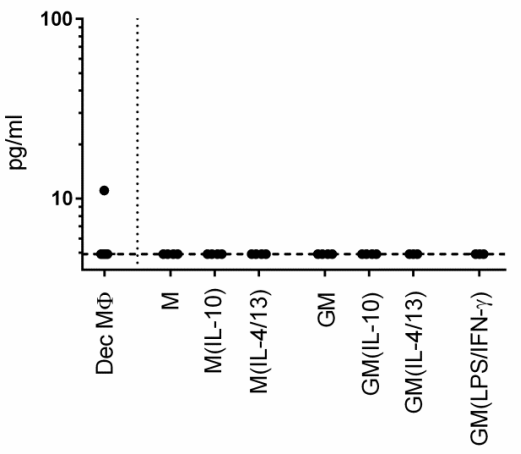

B.

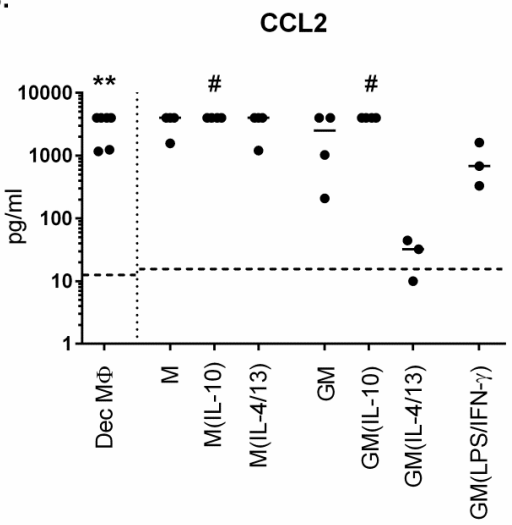

D.

$$
\text { CCL22 }
$$

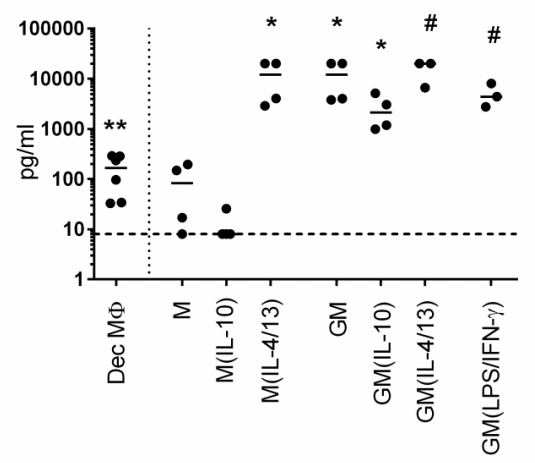

F.

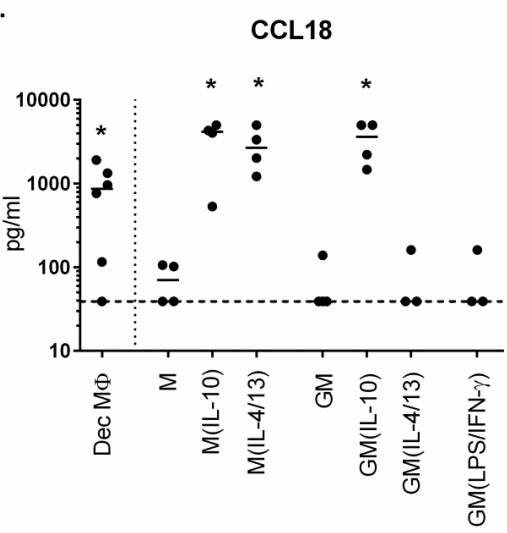



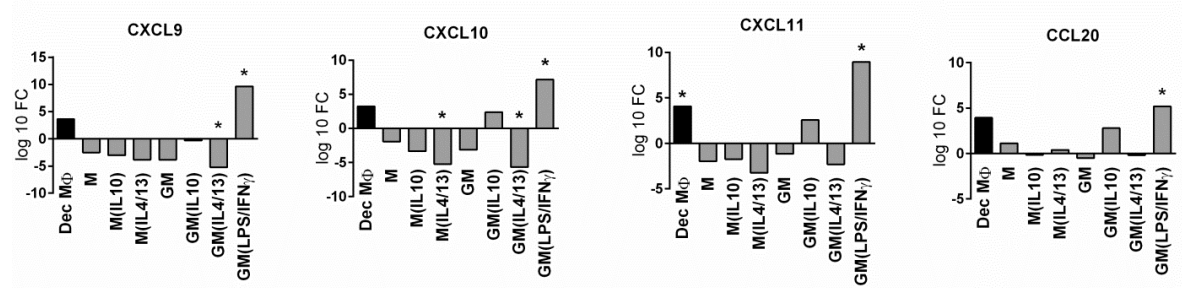

Th2
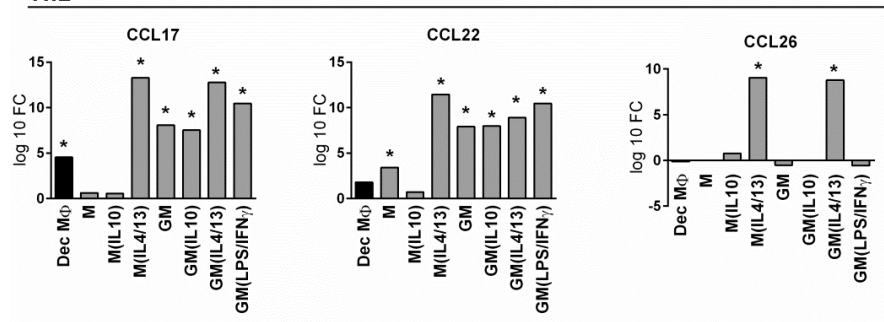

Th2 / Treg

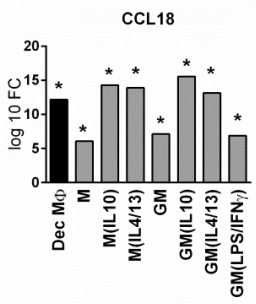

Myeloid
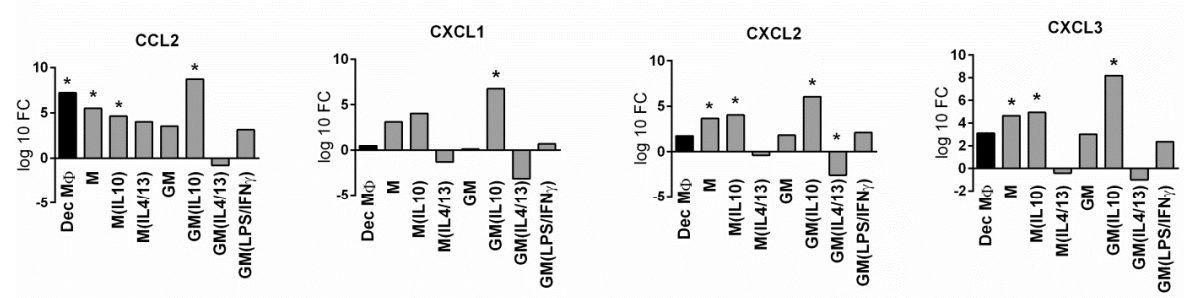

Myeloid

Myeloid / Treg

\section{Myeloid / NK cell}

B cell
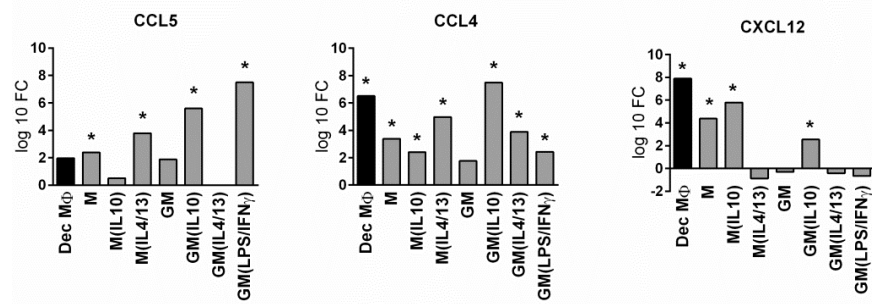

CXCL13

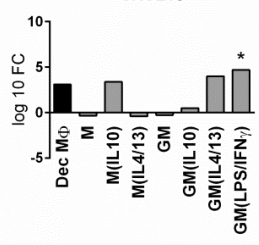

Figure 21. Expression of selected chemokine transcripts in decidual macrophages and different in vitro macrophage subsets. Chemokines are categorized under the major cell types for which they have recruitment specificity. Decidual macrophages and blood monocytes were isolated from first trimester human pregnancies and in vitro macrophages were generated from $\mathrm{CD}_{14}{ }^{+}$blood monocytes from non-pregnant women $(\mathrm{n}=3)$. Fold changes $(\log$ $10 \mathrm{FC}$ ) in respect to blood monocytes are shown. Genes where considered significantly regulated $(*)$ when $\mathrm{p} \leq 0.05$ and the fold change regulation was at least $+/-2$. M: M-CSF, GM: GM-CSF, Dec MФ: decidual macrophages. 
GM(LPS/IFN- $\gamma$ ) macrophages showed different chemokine patterns (Fig. 20). These data was largely confirmed by the analysis of a larger group of chemokines by gene expression analysis with microarray (Fig. 21 and paper II, Fig. 6A). Our results suggest that decidual macrophages contribute to maintain high numbers of macrophages and Treg cells, while limiting recruitment of several lymphocyte subsets to the decidua.

By using M(IL-10) macrophages as a model of decidual macrophages (which showed similar chemokine gene and protein patterns, Fig. 20 and 21 and paper II, Fig. 6A), we show that these cells preferentially promote the recruitment of $\mathrm{CD} 14^{+}$monocytes, possibly mediated by CCL2 (Fig. 22). Mouse studies showing the CCR2-dependent recruitment of macrophages to the pregnant uterus are in support of a CCL2-driven recruitment mechanism (Tagliani et al., 2011). Although it is possible that CCL18 may contribute to Treg cell recruitment in vivo, CCL18-producing M(IL-10) macrophages failed to induce migration of Treg cells in our chemotaxis model (Fig. 22). Since these results are based on an in vitro model using M(IL-10) macrophages, the role of decidual macrophages in the recruitment of decidual leukocytes requires further investigation. In addition to their primary recruiting potential, chemokines are involved in several other functional processes. In this regard, the ability of CCL2 to
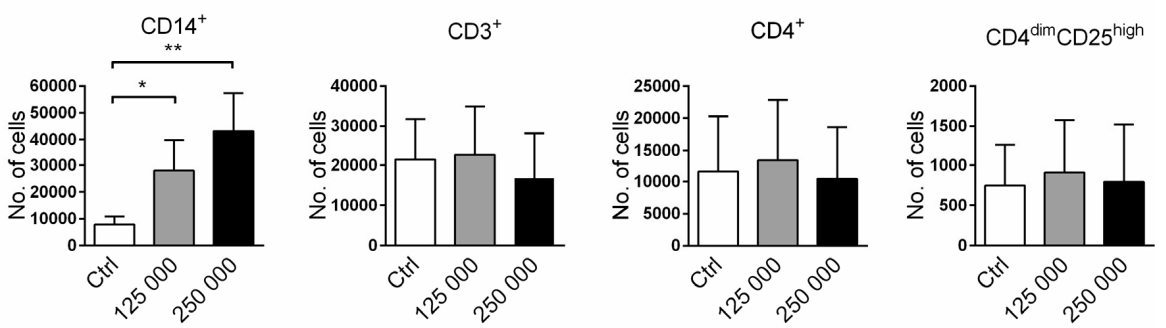

Figure 22. M-CSF- and IL-10-stimulated macrophages selectively recruited CD14 ${ }^{+}$ monocytes. Macrophages were generated from $\mathrm{CD} 14^{+}$blood monocytes from non-pregnant women. CFSE-labeled macrophages (125000 or 250000 ) were placed in the lower wells and PBMC in the upper wells of a Transwell plate and cells were allowed to migrate overnight. Recruited cells were identified as $\mathrm{CFSE}^{-}$and were characterized by flow cytometry. The graphs show the mean $+\mathrm{SD}$ of six experiments. $* \mathrm{p} \leq 0.05, * * \mathrm{p} \leq 0.01$ (versus medium control). Ctrl: medium control, PBMC: peripheral blood mononuclear cells. 
promote M2 polarization (Sierra-Filardi et al., 2014) and of CCL18 to promote induction of Treg cells (Chang et al., 2010; Melief et al., 2013), polarization of M2 macrophages (Schraufstatter et al., 2012) and tolerogenic DCs (Azzaoui et al., 2011), makes them potential candidates in the maintenance of fetal-maternal tolerance by promoting a tolerogenic leukocyte environment.

Decidual macrophages are prominent chemokine producers and therefore, failure to acquire M2 characteristics and accordingly, increased production of inflammatory chemokines, may be associated with the development of complications. CXCR3 ligands have been of particular interest because of their involvement in Th1-associated responses, which are incompatible with normal fetal development. For instance, CXCR3-mediated recruitment of T cells during Listeria monocytogenes infection in mice resulted in fetal loss (Chaturvedi et al., 2015). Interestingly, decidual stromal cells did not account for the increased production of the CXCR3 ligand CXCL9, supporting previous data where decidual stromal cells fail to upregulate CXCL9 and CXCL10 upon inflammatory stimuli (Nancy et al., 2012). In contrast, infiltrating macrophages and neutrophils were shown to be responsible for this inflammatory chemokine production (Chaturvedi et al., 2015). The observation that isolated human decidual macrophages fail to upregulate CXCL10 upon TLR-ligation in vitro (Duriez et al., 2014) supports the idea that newly recruited monocytes, rather than "resident" or differentiated decidual macrophages, may differentiate into Th1 chemokineproducing M1 macrophages.

Increased chemokine production during labor (Hamilton et al., 2013) and increased local and circulating levels of CXCL10 and CXCL11 in preeclampsia (Gotsch et al., 2007; Boij et al., 2012; Lockwood et al., 2013) suggests that altered chemokine production may be associated with both normal (in the case of labor) and pathological inflammatory responses also in the absence of infections. Thus, failure to maintain an M2 phenotype and a shift towards M1 polarization with increased production of inflammatory, in particular Th1-recruiting, chemokines could contribute to the accumulation of inflammatory leukocytes commonly associated with adverse pregnancy outcomes. 


\section{Markers of polarized macrophage subsets}

Although not the principal aim in papers I and II, the comparison of a wide panel of cell surface markers, cytokines, chemokines and genes across the different in vitro polarized macrophage subsets, provided the opportunity to evaluate the suitability of, and to identify new, subset-specific macrophage markers. Some of these markers are discussed below.

Expression of CD163 is often associated with M2, including both homeostatic and Th2-associated, macrophages (Biswas and Mantovani, 2010). Our results showed however that CD163 is mainly restricted to M-CSF- and IL-10-induced macrophages, since not only LPS/IFN- $\gamma$ but also IL-4/IL-13 downregulated CD163 expression (Fig. 14), making CD163 a potential marker of homeostatic macrophages. This is supported by studies evaluating the effects of M-CSF versus GM-CSF and the additional effects of IL-10, IL-4 and IFN- $\gamma$ (Ambarus et al., 2012; Kittan et al., 2013). Importantly, CD163 is also expressed on many tissue macrophages that predominantly have homeostatic functions (Van den Heuvel et al., 1999). CD209 is another marker commonly used to define M2 macrophages and our results showed that it is induced by both homeostatic and Th2 stimuli while it is downregulated by pro-inflammatory stimuli (Fig. 14), in line with previous data (Soilleux et al., 2002; Puig-Kroger et al., 2004; Dominguez-Soto et al., 2011). CD209 may be used as a general M2 marker, but additional markers should be used to define the specific subtype. In contrast, NRP-1 that was proposed to be an M2 marker because of its upregulation by M-CSF and dowregulation by IFN- $\gamma$ (Ji et al., 2009), was highly expressed on all macrophage subsets analyzed in paper I (Fig. 14). However, by evaluating the relative expression (MFI) among subsets, the high NRP-1 expression on IL-4/IL-13 macrophages suggests that it may be more associated with Th2 responses (paper I, supplemental Table I).

The transcription factor IRF5 has been proposed to promote M1 macrophages with production of IL-12 and IL-23 and to inhibit M2 polarization and IL-10 production (Krausgruber et al., 2011). In paper I, we could confirm that GM(LPS/IFN- $\gamma$ ) 
macrophages expressed high levels and decidual macrophages low levels of IRF5; however, the pattern of expression was not entirely consistent with an M1-promoting and M2-inhibiting function (paper I, Fig. 6A). Although the original suggestion of IRF5 as an M1 marker was based on M-CSF- and GM-CSF-driven macrophages, more recent data show that both M-CSF and GM-CSF upregulate IRF5 (Lacey et al., 2012). In addition, IL-4-stimulated macrophages were shown to express higher levels of IRF5 than LPS/IFN- $\gamma$-stimulated macrophages (Jaguin et al., 2013), supporting our data and questioning the suitability of this transcription factor as an M1 marker.

Also, the distinct chemokine profiles observed among in vitro macrophages may be useful for the classification of different macrophage subsets as has been previously suggested (Mantovani et al., 2004). High production of CXCL9-11 was, as expected, a characteristic associated with GM(LPS/IFN- $\gamma$ ) or M1 macrophages (Fig. 20 and 21), which is in line with other reports (Mantovani et al., 2004; Jaguin et al., 2013; Kittan et al., 2013). Production of the Th2-related chemokines CCL17 and CCL22 was however not restricted to IL-4/IL-13-stimulated macrophages as anticipated, but was also induced in response to GM-CSF and GM-CSF in combination with LPS/IFN- $\gamma$ (Fig. 20 and 21). In contrast, CCL26 that is an eosinophil-recruiting chemokine (Blanchard et al., 2006) emerged as a potential chemokine specific for Th2 immunity (Fig. 21). Further, homeostatic macrophages, in particular IL-10-induced, may be characterized by the combined high production of CCL2 and CCL18, and low production of both Th2- and Th1-associated chemokines (CCL17, CCL22, CCL26, CXCL9-11) (Fig. 20 and 21). 


\section{Role of the placenta in promoting a homeostatic immune environment}

\section{Induction of homeostatic M2 macrophages and Treg cells}

It is fairly settled that decidual immune cells adopt immune regulatory properties and that these are essential for a healthy pregnancy outcome. However, the mechanisms behind this adaptation in humans are largely unknown. Since the placenta is a temporary organ closely associated with decidual leukocytes, we hypothesized that it could have a major influence on decidual leukocyte adaptation. The placenta and trophoblast cells have indeed been described to have a wide range of immune regulatory properties; still the ability of the human placenta and the relative contribution of placental-derived factors in promoting tolerogenic leukocytes have not been addressed in a physiological setting. Therefore, in paper III we evaluated the effects of placental- and trophoblast-derived factors on the differentiation and polarization of the major decidual leukocyte populations with regulatory functions, i.e. macrophages, Th cells and Treg cells.

By using conditioned medium from first trimester placental tissue during the in vitro differentiation of macrophages, we showed that placental factors can induce macrophages that express CD14,CD163, CD206 and CD209 and produce IL-10 and CCL18 but not IL-12 and IL-23, i.e. resembling decidual macrophages (Fig. 23). We further showed by using neutralizing antibodies that M-CSF and, in particular, IL-10 were involved in the M2 polarization process (paper III, Fig. 6A-B). As discussed earlier, these results highlight the involvement of M-CSF and IL-10 in the regulation of decidual macrophage differentiation and polarization. These data also identified the placenta as an important source of M-CSF (whose levels greatly exceeded the levels of GM-CSF) and of IL-10, and both factors were mainly expressed by CTB cells and syncytiotrophoblasts (paper III, Fig. 5 and Table I). However, in addition to the placenta, several other cells (including decidual stromal cells, NK cells, Th cells and 

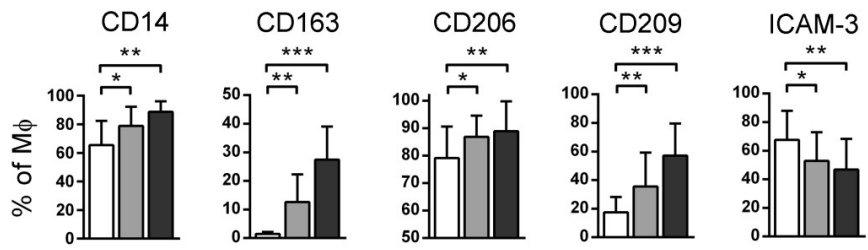

$\square \mathrm{GM}$

$\mathrm{GM}+6.25 \% \mathrm{PE} C \mathrm{M}$

$\mathrm{GM}+12.5 \% \mathrm{PE} \mathrm{CM}$
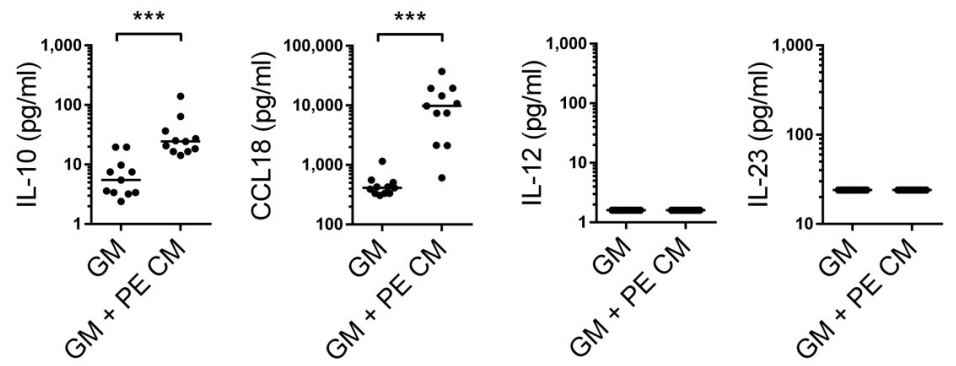

Figure 23. Soluble factors from placental tissue induce macrophages with homeostatic properties. Placental explant conditioned medium (PE CM) induced expression of the macrophage markers CD14, CD163, CD206 and CD209, and reduced ICAM-3 expression in a dose-dependent manner. PE CM (12.5\%) also induced the production of IL-10 and CCL18 from macrophages, while IL-12 and IL-23 were not induced. Macrophages were generated from $\mathrm{CD} 14^{+}$monocytes by culturing them for six days in the presence of $5 \mathrm{ng} / \mathrm{ml} \mathrm{GM-CSF}$ (GM) alone or in combination with PE CM. The graphs show the mean + SD from 12 individual experiments. ${ }^{*} \mathrm{p} \leq 0.05,{ }^{* *} \mathrm{p} \leq 0.01,{ }^{* *} \mathrm{p} \leq 0.001$. МФ: macrophages.

macrophages themselves) can produce M-CSF and IL-10 (Hatayama et al., 1994; Piccinni et al., 2001; Lidstrom et al., 2003; Engert et al., 2007) and may further influence the M2 polarization of decidual macrophages.

In order to mimic the decidual microenvironment at the resting state, $\mathrm{CD} 4^{+} \mathrm{T}$ cells were cultured with PE CM without any additional stimulation ("unstimulated"). In this way, we showed that placental factors could promote the preferential expansion of Treg cells (defined as Foxp $3^{+}, \mathrm{CD} 4{ }^{\mathrm{dim}} \mathrm{CD} 25^{\text {high }}$ or $\mathrm{CD} 25^{\text {high }}$ Foxp $3^{+}$) and increase the production of IL-10 by CD4 ${ }^{+}$T cells (Fig. 24 "unstim", and paper III, Fig. 3A). The expanded Treg cell population was $\mathrm{CD} 127^{\text {low }}$, expressed the suppressive markers CTLA-4 and CD39, and was predominantly CD45R0 $0^{+}$(paper III, Fig. 3C), 

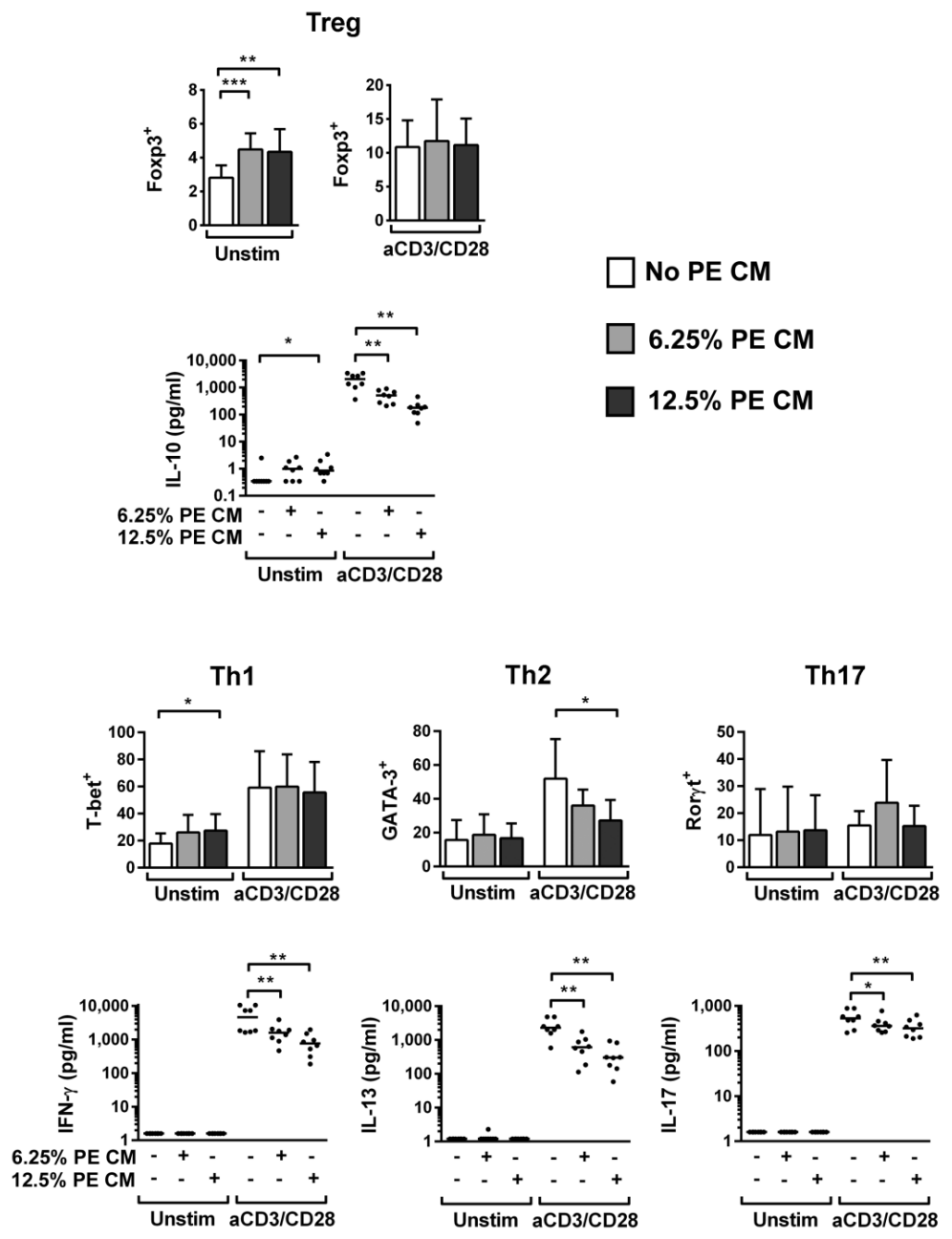

Figure 24. Soluble factors from placental tissue preferentially induce Foxp3 expression and production of IL-10 in $\mathrm{CD}^{+} \mathrm{T}$ cells. The graphs show the effect of $6.25 \%$ and $12.5 \%$ first trimester placental explant conditioned medium (PE CM) on the polarization of Treg, Th1, Th2 and Th17 cells. The bar graphs show the expression of the transcription factors Foxp3 (Treg), T-bet (Th1), GATA-3 (Th2) and Roryt (Th17) in the CD4 ${ }^{+} \mathrm{T}$ cell population and the scatter plots show the production of cytokines from the corresponding Th cell subset.

Peripheral blood $\mathrm{CD}^{+} \mathrm{T}$ cells were cultured either unstimulated (Unstim) or stimulated with $0.25 \mu \mathrm{g} / \mathrm{ml}$ anti-CD3 and anti-CD28 antibodies (aCD3/CD28) for five days in the presence or absence of PE CM. The graphs show the mean $+\mathrm{SD}$ (bar graphs) or the median (scatter plots) of eight individual experiments. $* \mathrm{p} \leq 0.05,{ }^{* *} \mathrm{p} \leq 0.01,{ }^{* * *} \mathrm{p} \leq 0.001$. 
thus resembling decidual Treg cells (Tilburgs et al., 2008; Mjosberg et al., 2010). Further, we showed that the placental-induced Treg cells were functionally suppressive in vitro, by evaluating their effect on the proliferation of CFSE-labeled and anti-CD3/CD28-stimulated responder cells (paper III, Fig. 3D-E). Expression of the Th1-associated transcription factor T-bet was also increased by placental-derived factors, but this increase was not paralleled by increased production of IFN- $\gamma$ by CD4 ${ }^{+}$ T cells (Fig. 24). In contrast, expression of GATA-3 and Roryt and production of IL13 and IL-17, associated with Th2 and Th17 cells respectively, were not induced. The selective increase of Treg cells is in line with their reported accumulation in the human decidua (Tilburgs et al., 2006; Mjosberg et al., 2010) and with the importance of Treg cells in murine pregnancy (Aluvihare et al., 2004; Rowe et al., 2012b; Samstein et al., 2012). Although the increase in T-bet ${ }^{+}$cells was unexpected, a high frequency of CCR6 $6^{-}$Th 1 cells, associated with moderate production of IFN- $\gamma$, has been reported in the human decidua (Mjosberg et al., 2010), thus supporting a role of the placenta in shaping the decidual microenvironment.

An increasing number of in vitro studies clearly demonstrate the important bidirectional communication among decidual cells that results in the development of tolerogenic leukocytes. For example, decidual macrophages are able to inhibit NK cell cytotoxicity (Co et al., 2013), to suppress T cell proliferation and activation (Mizuno et al., 1994; Sayama et al., 2013) and to induce Treg cells (Vacca et al., 2010; Hsu et al., 2012), which our results further support. Also decidual stromal cells have been shown to contribute to limited T cell activation and induction of Treg cells (Nagamatsu et al., 2009; Erkers et al., 2013). Although, to our knowledge, the function of human decidual Treg cells has not been studied, the ability of Treg cells to induce $\mathrm{CD} 163^{+} \mathrm{CD} 206^{+} \mathrm{CCL} 18^{+} \mathrm{M} 2$ macrophages and macrophages with limited proinflammatory potential in vitro (Taams et al., 2005; Tiemessen et al., 2007), suggests that also decidual Treg cells may promote M2 macrophages. Nevertheless, since our results indicate that placental-derived factors can promote homeostatic macrophages and Treg cells, we hypothesize that the placenta itself, being a newly developed organ, is the primary trigger of maternal immune cell adaptation. We also suggest that the crosstalk between the induced tolerogenic leukocytes is an essential secondary 


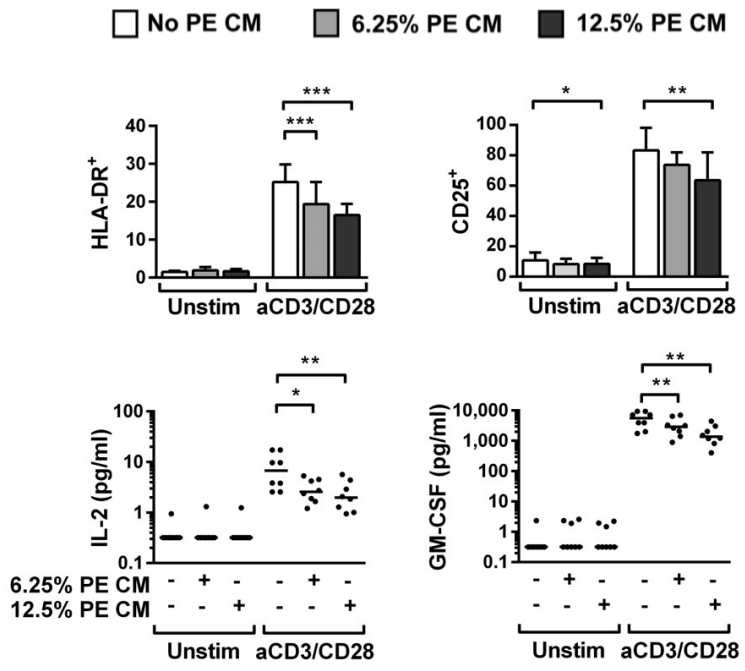

Figure 25. Effect of $6.25 \%$ and $12.5 \%$ placental explant conditioned medium (PE CM) on the activation of $\mathrm{CD}^{+} \mathrm{T}$ cells. Peripheral blood $\mathrm{CD} 4^{+} \mathrm{T}$ cells were cultured either unstimulated (Unstim) or stimulated with $0.25 \mu \mathrm{g} / \mathrm{ml}$ anti-CD3 and anti-CD28 antibodies (aCD3/CD28) for five days in the presence or absence of PE CM. The graphs show the mean $+\mathrm{SD}$ (bar graphs) or the median (scatter plots) of eight individual experiments. ${ }^{*} \mathrm{p} \leq 0.05, * *$ $\mathrm{p} \leq 0.01, * * * \mathrm{p} \leq 0.001$.

mechanism to sustain the homeostatic uterine environment induced by the placenta. The influence of the placenta on tolerogenic decidual leukocytes is likely not restricted to macrophages and Th cells. For, example it was recently reported that trophoblast-derived thymic stromal lymphopoietin (TSLP) could promote DCs with tolerogenic properties, for instance with high production of IL-10 and TGF- $\beta$ and low production of IL-12 and TNF (Du et al., 2014a). In turn, these DCs induced the conversion of functionally suppressive $\mathrm{CD} 4^{+} \mathrm{CD} 25^{+} \mathrm{Foxp}^{+}$Treg cells that also produced IL-10 and TGF- $\beta$.

Placental-derived factors also caused a general downregulation of Th cell activation in anti-CD3/CD28-stimulated cells, as shown by for example decreased levels of HLADR and CD25 and a generally reduced production of cytokines, including those 
associated with Th1, Th2 and Th17 immunity (Fig. 24 and 25, “aCD3/CD28”). We interpreted these data as the ability of the placenta to limit Th cell activation regardless of the type of immune response. Similar to the effect of placental-derived M-CSF and IL-10 on macrophage polarization, we suggest that the placenta promotes Th cells with an increased threshold of activation, which may be essential for preserving a homeostatic decidual immune environment. Bearing in mind that fetal rejection might not only be caused by activation of placental/fetal-specific T cells but also by general immune cell activation, for instance during infections (Krishnan et al., 1996; Rowe et al., 2012a), the ability of the placenta to induce immune cells with a reduced inflammatory potential might be essential for maintaining tissue integrity and preventing inflammation-induced fetal rejection.

Our data also showed that conditioned medium from isolated CTB and EVT cells could induce the phenotype of decidual macrophages and the expansion of Treg cells (Fig. 26A and C), indicating trophoblast cells as major inducers of decidual immune cell adaptation. Notably, IL-10 that was involved in both M2 macrophage and Treg cell polarization was produced at particularly high levels by isolated CTB and EVT cells (paper III, Fig. 5B and Table I). Although the major cellular components of the placenta are trophoblast cells, other cells are present in the placental villous core and could potentially promote immune cells with regulatory functions. For example, placental mesenchymal stem cells have been shown to promote M2 macrophages $\left(\mathrm{CD}_{163}{ }^{+} \mathrm{CD} 206^{+} \mathrm{IL}-10^{+} \mathrm{IL}-12^{-}\right)$and to limit T cell activation (Chang et al., 2006; Abumaree et al., 2013). However, considering that trophoblast cells are in closer association with decidual leukocytes, we suggest that these are the major placental cell type influencing decidual immune cells. Mesenchymal stem cells, together with trophoblast cells, are perhaps more likely to influence the polarization of fetal placental macrophages (Hofbauer cells), which show many similarities with decidual macrophages (Bockle et al., 2008; Joerink et al., 2011). 

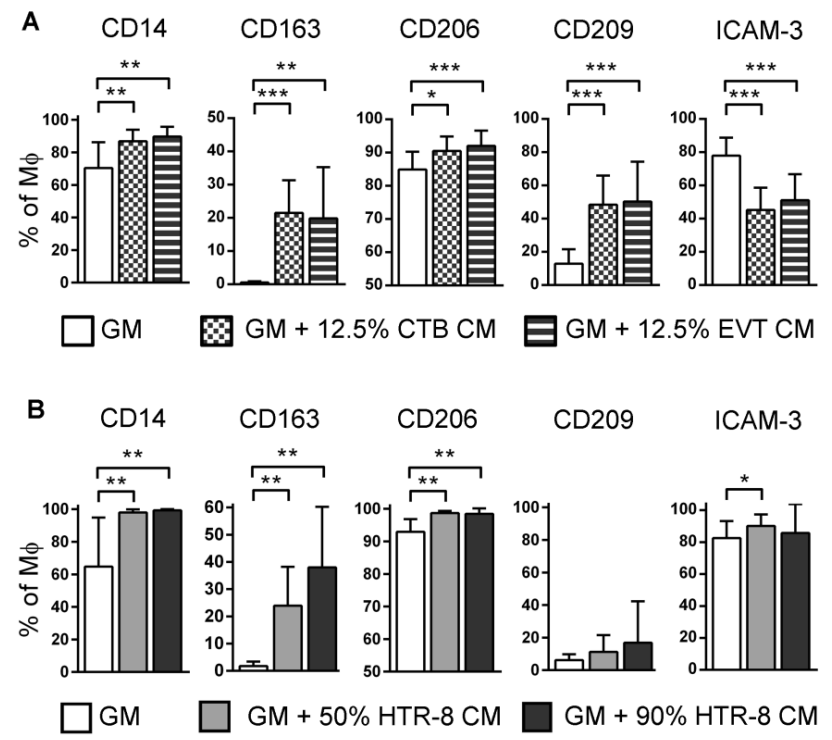

C $\mathrm{CD} 25^{\text {high }} \mathrm{Foxp}^{+}$

D CD25 $^{\text {high }}$ Foxp3 $^{+}$
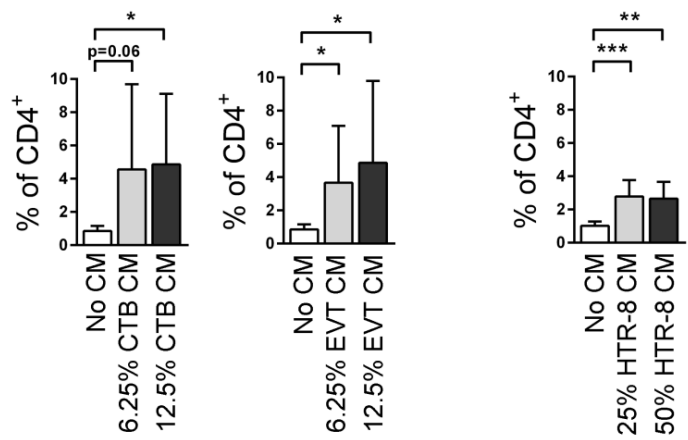

Figure 26. Effect of conditioned medium (CM) from (A) isolated first trimester placental cytotrophoblast (CTB) and extravillous trophoblast (EVT) cells or (B) the trophoblast cell line HTR-8/SVneo (HTR-8) on macrophage cell surface markers. Effect of (C) CTB or EVT CM or (D) HTR-8 CM on CD $25^{\text {high }}$ Foxp $3^{+}$Treg cells. (A and B) Macrophages were generated from $\mathrm{CD} 14^{+}$monocytes (isolated from non-pregnant women) by culturing them for six days with GM-CSF (GM) alone or in combination with CM. (C and D) CD4 ${ }^{+} \mathrm{T}$ cells (isolated from non-pregnant women) were cultured for five days with $\mathrm{CM}$ at the indicated concentrations. All graphs show the mean + SD from nine individual experiments. ${ }^{*} \mathrm{p} \leq$ $0.05, * * \mathrm{p} \leq 0.01, * * * \mathrm{p} \leq 0.001$. MФ: macrophages. 


\section{The HTR-8/SVneo cell line differs from primary trophoblast cells}

In paper III, we also evaluated the effects of conditioned medium from the first trimester trophoblast cell line HTR-8/SVneo, which is commonly used as a substitute for primary trophoblast cells. Although HTR-8/SVneo cells in some ways behaved similar to placental tissue and CTB and EVT cells, they also differed in several aspects. For instance, HTR-8/SVneo conditioned medium failed to induce upregulation of CD209 (Fig. 26B). Further, HTR-8/SVneo cells enhanced the activation of anti-CD3/CD28-stimulated Th cells by for instance upregulating HLADR and both transcription factors and cytokines associated with Th1, Th2 and Th17 cells (paper III, supplemental Fig. 2). These results were in contrast to the downregulating effects of placental conditioned medium on activated Th cells. In addition, the concentrations required to induce the M2 phenotype in macrophages and the expansion of Treg cells were considerably higher for HTR-8/SVneo than for CTB and EVT cells (Fig. 26). A clear difference from primary trophoblast cells was the lack of IL-10 production by HTR-8/SVneo cells (paper III, Fig. 5B and Table I), which is likely an important explanation to the different effects on both macrophages and Th cells. Notably, HTR-8/SVneo cells instead produced considerably higher levels of TGF- $\beta 1$ and TGF- $\beta 2$ than did CTB and EVT cells (paper III, Fig. 5C and Table I), possibly accounting for some of the regulatory effects observed. Our data is in line with other studies showing that HTR-8/SVneo cells, like several other cell lines, may not be representative of primary trophoblast cells (King et al., 2000c; Apps et al., 2009; Morales-Prieto et al., 2012).

\section{Factors involved in the expansion of Treg cells}

Although Treg cells have been extensively studied in the context of pregnancy, the factors responsible for the specific enrichment of Treg cells at the fetal-maternal interface in humans are still unknown. However, due to their essential role in the establishment and maintenance of pregnancy, a redundancy of factors is to be expected. In paper III, by using neutralization antibodies, we showed that placentalderived IL-10, TGF- $\beta$ and TNF-related apoptosis-inducing ligand (TRAIL) partially 
contributed to the Treg cell induction (paper III, Fig. 6C-E). These factors have been shown to promote Treg cell expansion by different mechanisms. For example, TGF- $\beta$ promotes the conversion of naïve $\mathrm{CD}^{+} \mathrm{T}$ cells into Foxp3-expressing Treg cells (Chen et al., 2003; Rao et al., 2005). In contrast, the apoptosis-inducing factor TRAIL may promote the preferential expansion of an already existing Treg cell population while inhibiting expansion of non-Treg cells, as shown in a mouse model (Ikeda et al., 2010). IL-10 may also promote the preferential survival of Treg cells by specifically upregulating the anti-apoptotic Bcl-2 in Treg cells but not in conventional T cells (Santner-Nanan et al., 2013). Although the relevance of such mechanism, i.e. the preferential induction of apoptosis in non-Treg cells by TRAIL and IL-10, remains to be confirmed, it is in line with the established role of trophoblast-derived FasL and IDO in eliminating activated T cells (Hunt et al., 1997; Munn et al., 1998), which also should be in favor of an increased Treg cell proportion.

Other factors proposed to be involved in the generation of Treg cells are LIF and CCL18 (Gao et al., 2009; Chang et al., 2010). However, neither anti-LIF nor antiCCL18 neutralizing antibodies had an effect on the expansion of Treg cells induced by PE CM (data not shown). Still other placental-derived factors, for example soluble HLA-G, galectin-1 and pregnancy-associated hormones, (Blois et al., 2007; Selmani et al., 2008; Schumacher et al., 2013; Schumacher et al., 2014), may influence the generation of Treg cells. Together with the ability of decidual macrophages to induce Treg cells, as discussed previously, these placental-derived factors may be part of a redundancy of mechanisms that may ensure that homeostasis and fetal tolerance is maintained to protect the fetus. 


\section{Summary and conclusions}

The semi-allogeneic nature of the fetus implicates a great challenge during pregnancy, since the maternal immune system needs to adapt in order to tolerate the fetus without compromising the ability to protect the mother and the fetus against infections. Increasing evidence suggests that decidual macrophages and Treg cells have an important role in creating an immune environment that is compatible with normal fetal development. However, although the function of Treg cells is well established, the function of decidual macrophages has not been thoroughly investigated. Further, the factors involved in the regulation of decidual macrophages and Treg cells in humans are largely unknown.

In paper I and II, we showed that decidual macrophages from first trimester human pregnancy have characteristics mainly associated with homeostatic functions (summarized in figure 27). They expressed pattern recognition receptors (CD163, CD206 and CD209) and produced predominantly immune suppressive cytokines, including IL-10 and IL-35, while production of pro-inflammatory cytokines (for instance IL-12, IL-23 and TNF) was limited. These data suggest that decidual macrophages may contribute to the protection against infections while preserving a homeostatic uterine environment. Decidual macrophages also promoted the expansion of CD $25^{\text {high }}$ Foxp $^{+}$Treg cells but not of T-bet ${ }^{+}$Th1, GATA- $3^{+}$Th2 or Ror $\gamma \mathrm{t}^{+}$Th17 cells, suggesting that they contribute to establish fetal tolerance. Further, we showed that decidual macrophages are a prominent source of chemokines, implicating them as major regulators of cell trafficking at the fetal-maternal interface. In particular, they produced high levels of the monocyte- and Treg cell-associated chemokines CCL2 and CCL18, while Th1-, Th2- and Th17-recruiting chemokines were produced at low levels. Decidual macrophages could therefore contribute to shape the unique decidual leukocyte composition by maintaining high numbers of macrophages and Treg cells, while limiting recruitment of several lymphocyte subsets to the decidua. 


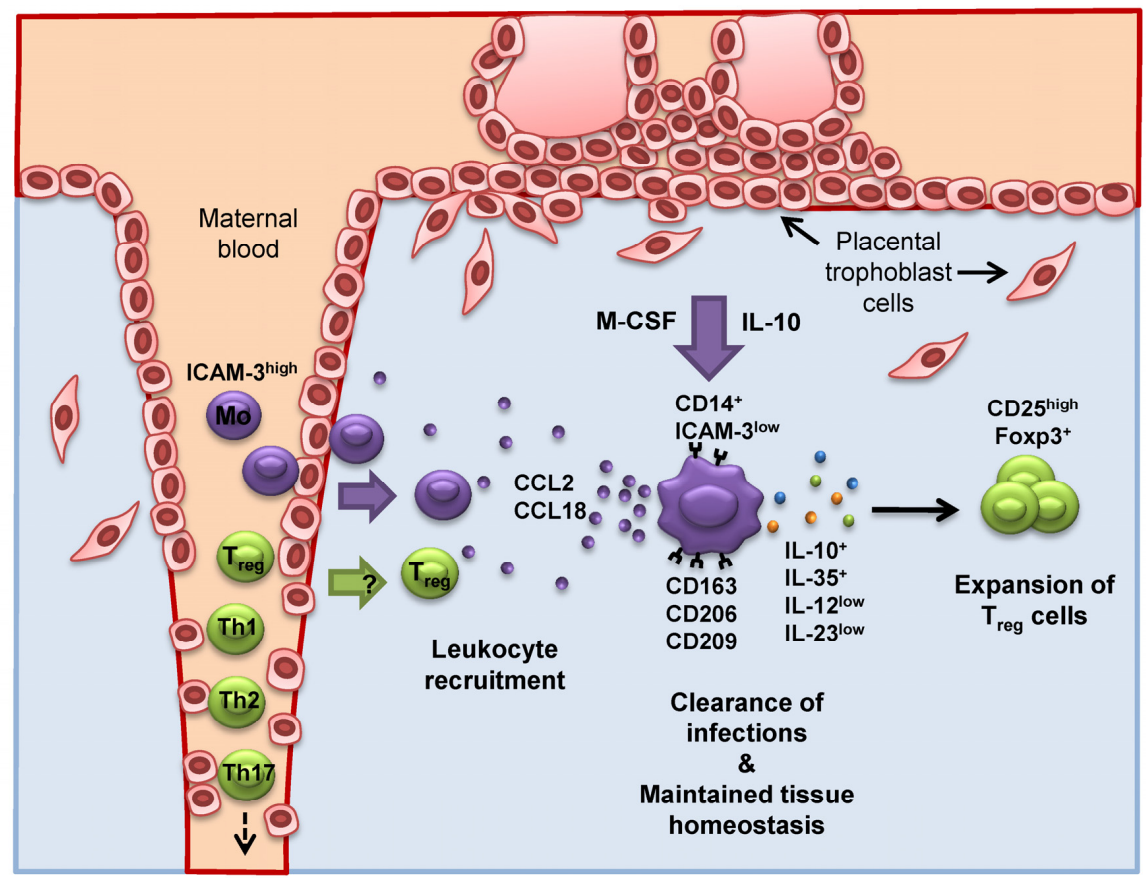

Figure 27. Proposed regulation and function of decidual macrophages during early human pregnancy. Locally produced M-CSF and IL-10 may contribute to the polarization of homeostatic M2 macrophages. Decidual macrophages may protect the fetus against infections while maintaining a homeostatic uterine environment, for instance by producing suppressive cytokines and by inducing Treg cells. Decidual macrophages may also contribute to the unique decidual leukocyte composition by selective recruitment of monocytes and Treg cells, while limiting migration of other lymphocytes into the decidua.

In papers I and II, we also showed that M-CSF and IL-10, but not Th1 and Th2 stimuli, induced macrophages that resemble decidual macrophages in terms of cell surface marker expression, cytokine and chemokine production and gene expression. Further, in paper III, placental tissue and placental trophoblast cells were identified as an important source of M-CSF and IL-10. These factors may therefore play an important role in the regulation of decidual macrophages during early human pregnancy. 
In paper III, we demonstrated that human fetally derived placental tissue promoted the induction of homeostatic M2 macrophages $\left(\mathrm{CD} 163^{+} \mathrm{CD} 206^{+} \mathrm{CD} 209^{+} \mathrm{IL}-10^{+} \mathrm{CCL} 18^{+}\right.$) and the selective expansion of functionally suppressive CD $25^{\text {high }}$ Foxp $3^{+}$Treg cells (Fig. 28). The placenta also limited activation of Th cells, for instance by generally reduced cytokine production. Our data suggests that the placenta has a unique ability to induce immune cells with a reduced inflammatory potential, which might be essential for maintaining tissue integrity and preventing inflammation-induced fetal loss.

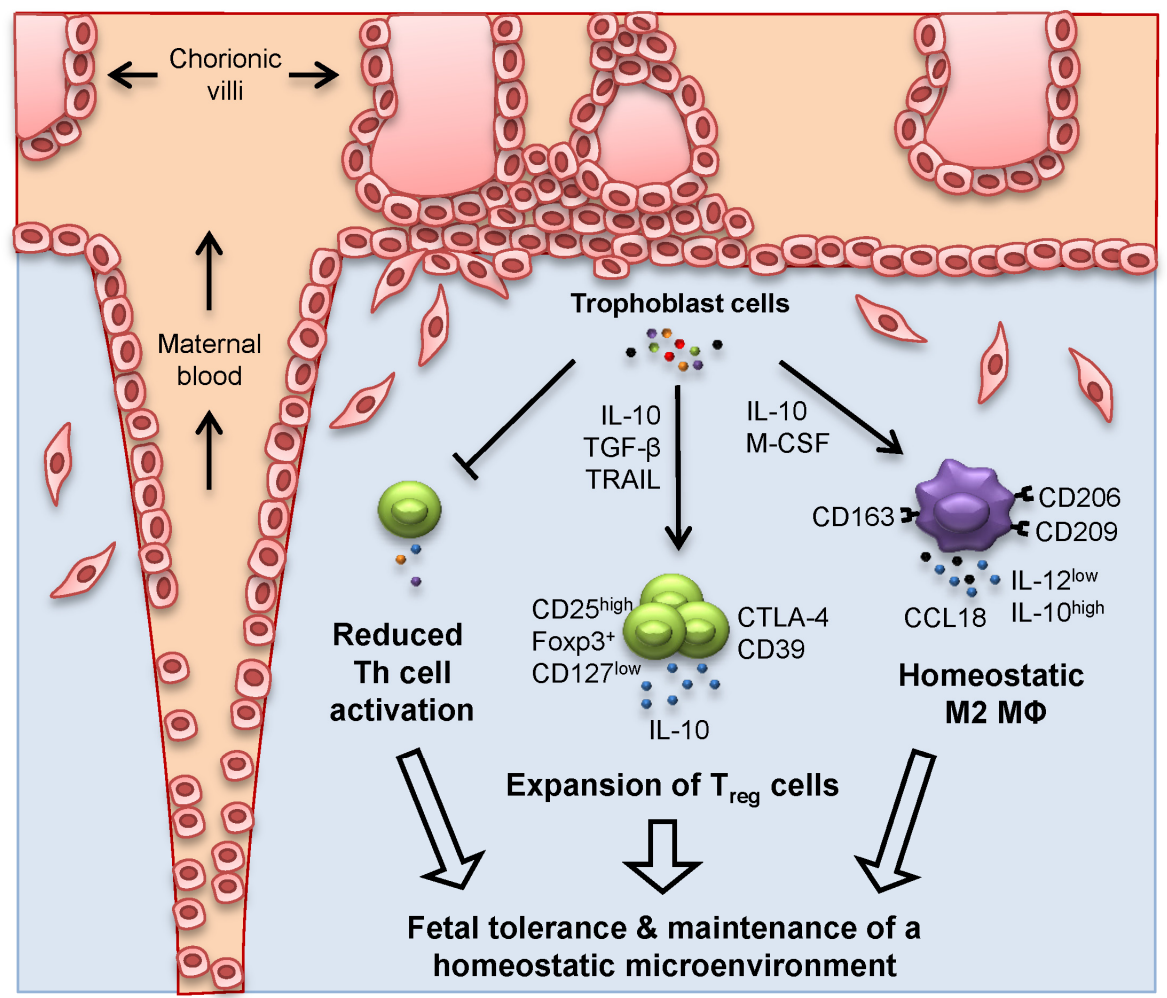

Figure 28. Influence of the placenta on the decidual microenvironment. Placental trophoblast cells may create a homeostatic and tolerant environment by producing factors, like M-CSF, IL-10, TGF- $\beta$ and TRAIL, that induce homeostatic macrophages (MФ) and regulatory $\mathrm{T}$ (Treg) cells and also limit excessive Th cell activation. We propose that the placenta is a primary inducer of tolerogenic immune cells thereby supporting normal fetal development. Figure from Svensson-Arvelund et al. (2015). 


\section{Future perspectives}

The data presented in this thesis may offer improved possibilities to further explore and understand the pathogenesis of pregnancy-associated complications. Furthermore, the identification of factors regulating normal physiology during pregnancy may be useful in the search for potential biomarkers in pregnancy complications. In addition, pregnancy can be used as a model for successful tolerance induction and regulation of inflammation, which should be of great value in areas of medicine characterized by unrestrained inflammation, such as autoimmune diseases and transplantation reactions.

However, several questions regarding the normal function of decidual macrophages remain unanswered. For example, due to their prominent ability to produce chemokines, the contribution of decidual macrophages to the recruitment of decidual leukocytes, both at the resting state and during infections, deserves to be further investigated, as does the role of decidual macrophages in the recruitment of placental trophoblast cells. Further, the interactions between decidual macrophages and other cells at the fetal-maternal interface is likely important for their function. It would therefore be valuable to analyze the properties of decidual macrophages in co-cultures with for instance NK cells, but also non-immune cells like decidual stromal cells and trophoblast cells.

Also the origin of decidual macrophages, which has implications for the response to infections, is an area that requires further study. Although decidual macrophages have been proposed to be continuously recruited from blood monocytes (Tagliani et al., 2011) a recent report suggested that a subpopulation of uterine macrophages may be of fetal origin (Zhao et al., 2015). In mice, infiltrating rather than tissue resident macrophages seem to account for the inflammatory response during infection in the uterus (Chaturvedi et al., 2015), but also in other conditions in other organs such as experimental autoimmune encephalomyelitis and colitis (Codarri et al., 2011; Bain et al., 2013). Thus, if decidual macrophages only derive from the continuous recruitment of monocytes, a pro-inflammatory milieu may cause the differentiation of M1 rather 
than M2 macrophages, quickly disrupting the homeostatic decidual environment. In contrast, longed-lived resident macrophages may be more resistant to a proinflammatory shift and may contribute to maintain a high threshold of activation as well as to a more rapid recovery after an inflammatory insult. Thus, an increased understanding regarding the origins of decidual macrophages would facilitate the extrapolation of results from isolated decidual macrophages to the in vivo situation.

An interesting finding was the ability of M-CSF to induce homeostatic M2 macrophages and of GM-CSF to counteract this effect. Several studies have analyzed the concentrations of these factors, especially GM-CSF, in pregnancy complications, however with inconsistent results (Greer et al., 1994; Gratacos et al., 1998; Hayashi et al., 2004; Huang et al., 2010; Boij et al., 2012). Given their dose-dependent and differential effects on macrophage polarization, studying the levels of M-CSF and GM-CSF in relation to each other, rather than analyzing them separately, should be more informative. It would also be of value to assess if circulating M-CSF and GMCSF concentrations mirror the levels at the fetal-maternal interface, for instance produced by decidual and placental explants from healthy pregnancy. In such case, an altered circulating M-CSF/GM-CSF ratio, reflecting an inflammatory uterine environment, could be used to predict adverse pregnancy outcomes. Moreover, due to their therapeutic potential (Hume and MacDonald, 2012; Hamilton and Achuthan, 2013), understanding the role of M-CSF and GM-CSF (and the possible role of IL-34) at the fetal-maternal interface is of relevance for the potential treatment of pregnancy complications.

Another potential biomarker is soluble CD163 (sCD163), which is shed from the cell surface of macrophages during inflammation and upon macrophage activation (Moller, 2012). Levels of sCD163 were for instance shown to be elevated in the cerebrospinal fluid of multiple sclerosis patients (Stilund et al., 2014) and in the serum of rheumatoid arthritis patients (Jude et al., 2013). In the context of pregnancy, increased serum sCD163 levels were shown to be associated with an increased risk of delivering preterm (Vogel et al., 2005). Since CD163-expressing decidual macrophages have been shown to be decreased in preeclampsia and spontaneous 
abortions (Schonkeren et al., 2011; Piao et al., 2015), it is possible that increased circulating sCD163 levels may also be found in these conditions, even before symptoms appear, reflecting a local shift from M2 to M1 macrophage activation. 


\section{Acknowledgements}

Jan Ernerudh, my main supervisor. Thanks for believing in me and for giving me so many great opportunities both within and outside my research projects. I really appreciate that you have let me be involved in all aspects of research from the very beginning; it has been a pleasure to discuss and plan all kinds of projects!

Maria Jenmalm, my co-supervisor, thanks for encouraging me and for always being up-to-date on basically everything research-related! Your optimism and ability to always see the possibilities are very much appreciated. Thanks for being so supportive also in issues outside research.

My co-supervisor Göran Berg, thanks for always being so kind and supportive and for providing with your clinical expertise. I appreciate that you always take the time to help me, even with short notice.

To my co-authors, Andreas Matussek, Robert Geffers and Sofia Freland, thanks for a fruitful collaboration. Ratnesh Bhai Mehta, thanks for all the help in paper III, and for sharing many lab hours taking care of samples. Heriberto RodriguezMartinez, thanks for the occasional but very much appreciated chats "en ca[h]tellano". A special thanks to Gendie Lash, for a very nice collaboration in paper III and other ongoing projects, and for giving me the opportunity to visit your lab in Newcastle.

To the staff at the Women's clinic (Ob/Gyn), thanks for the invaluable help on recruiting patients and collecting material for our studies. I also really appreciate your enthusiasm when we report about our research results!

Leif Matthiesen and Roland Boij, thank you for contributing with clinical perspectives in the GI-group. Special thanks to Leif for a very appreciated collaboration in "RSA-studien" and for your endless enthusiasm.

Jenny Mjösberg, thanks for being a great supervisor during my first project at AIR and for being a true inspiration! Charlotte Gustafsson, thanks for laying the foundation for my thesis and for your encouraging words at the very beginning and end of my PhD studies. 
Sandra Hellberg, you started as a shy student and then, what happened?! I have really appreciated your fun and crazy company in conferences (especially in Hamburg, where it all started...(-) but also for being the most well-organized person I know! Also, big thanks for taking the time to read through my thesis. Anna Forsberg, thanks for all the fun we have had both at work and outside work and for always taking initiative to social activities. Måns Edström, I really appreciate you for your relaxed attitude (almost all the time...(-)) and for being so genuinely nice!

Big thanks to Helena Enocsson, Linda Fryland and Måns Edström who started almost at the same time as me, finally I also finished! Marie Rubèr, Helena Enocsson, Linda Fryland and Lina Wirestam who I've mostly shared "doktorandrummet" with, thanks for all the fun talks over the years. A special thanks to Marie, who despite being so busy helped me with "RSA-studien" and to take care of samples during my parental leave and during the writing of this thesis. Daniel Eklund, another macrophage enthusiast, yeay! I really appreciate your eager to get things done. Klara Martinsson, I admire you for always dealing with things immediately (I have so much to learn...) Maria Svenvik, thanks for your positive spirit and for being a great shoe-shopping company in Budapest $(-)$.

Petra Cassel, Karin Söderman, Ammi Fornander, Marie Malander, Ylva Billing, Gunnel Almroth and Mari-Anne Åkesson, thanks for always being helpful and making things run smoothly at AIR. A special thanks to Gunnel that patiently helped taking (the very many) blood samples for me and my students. Karin, thanks for taking care of GRABB!

To other colleagues, Martina Abelius, Johanna Huoman, Hammoudi Alkaissi, Jaya Prakash Chalise, Sudeep Chenna Narendra, Jonas Wetterö, Thomas Skogh, Mattias Magnusson, Per Hultman, Christer Bergman, Alf Kastbom, Christina Ekerfelt, Said Havarinasab, Camilla Janefjord and all students that have contributed to the unusually open and pleasant atmosphere at AIR.

I am also very grateful to all the students that with so much enthusiasm worked on projects that not always went as planned: Robert Lindau, Sandra Hellberg, Daniel Söderberg, Valentina Bruno, Elahe Mirrasekhian, Caroline Wendel and Jennie Röjdner. Special thanks to Valentina, the most research-motivated and ambitious clinician I've met! Robert, I trust you can make the macrophages come back to life:-)

To the staff at the flow unit (at the blood central), thanks for always being so supportive, especially Karin Backteman that so patiently helped me solved all kinds of flow mysteries...even in your spare time! Adam and Evert Canto (and little Elof), thanks for always being there, without you this thesis would probably not exist. 
Ett stort tack till mina vänner (livet är ju inte bara jobb (-)), speciellt Ellen, Alexandra och Helene, jag uppskattar verkligen att vi fortfarande ses (om än lite för sällan)! Anna, tack för den härliga tid vi delade när du fortfarande bodde i Linköping och för att du är en sån go' vän! Barbara, jag är så himla glad att vi fortfarande håller ihop sen MedBi-tiden! Tack för allt roligt vi har hittat på genom åren. Royal, volleybolllaget som funnits under alla mina år som doktorand, tack till alla och speciellt till er som var med från allra första början, Oskar, Markus, Anders och Daniel (och Martin som tror att han var med från början... (;) för alla trevliga lagträffar genom åren!

Till min familj, mamma, Shera, Gustav och Jonas (+Alfred och Britta) tack för att ni alltid har stöttat mig, ni betyder väldigt mycket för mig!

Daniel, tack för att du alltid finns vid min sida och stöttar och uppmuntrar mig. Jag kan inte med ord beskriva hur mycket du betyder för mig! Alma, min lilla skatt, tack för att du förgyller min tillvaro! 


\section{References}

Abbas, A., Lichtman, A., and Pillai, S. (2015). Cellular and Molecular Immunology, 8th edn (Elsevier Saunders).

Abrahams, V.M., Visintin, I., Aldo, P.B., Guller, S., Romero, R., and Mor, G. (2005). A role for TLRs in the regulation of immune cell migration by first trimester trophoblast cells. J Immunol 175, 8096-8104.

Abumaree, M.H., Al Jumah, M.A., Kalionis, B., Jawdat, D., Al Khaldi, A., Abomaray, F.M., Fatani, A.S., Chamley, L.W., and Knawy, B.A. (2013). Human placental mesenchymal stem cells (pMSCs) play a role as immune suppressive cells by shifting macrophage differentiation from inflammatory M1 to anti-inflammatory M2 macrophages. Stem cell reviews 9, 620-641.

Acosta-Rodriguez, E.V., Rivino, L., Geginat, J., Jarrossay, D., Gattorno, M., Lanzavecchia, A., Sallusto, F., and Napolitani, G. (2007). Surface phenotype and antigenic specificity of human interleukin 17-producing T helper memory cells. Nat Immunol 8, 639-646.

Adolfsson, A., and Larsson, P.G. (2006). Cumulative incidence of previous spontaneous abortion in Sweden in 1983-2003: a register study. Acta Obstet Gynecol Scand 85, 741-747.

Akagawa, K.S., Komuro, I., Kanazawa, H., Yamazaki, T., Mochida, K., and Kishi, F. (2006). Functional heterogeneity of colony-stimulating factor-induced human monocyte-derived macrophages. Respirology 11 Suppl, S32-36.

Allan, S.E., Song-Zhao, G.X., Abraham, T., McMurchy, A.N., and Levings, M.K. (2008). Inducible reprogramming of human $\mathrm{T}$ cells into Treg cells by a conditionally active form of FOXP3. Eur J Immunol 38, 3282-3289.

Aluvihare, V.R., Kallikourdis, M., and Betz, A.G. (2004). Regulatory T cells mediate maternal tolerance to the fetus. Nat Immunol 5, 266-271.

Ambarus, C.A., Krausz, S., van Eijk, M., Hamann, J., Radstake, T.R., Reedquist, K.A., Tak, P.P., and Baeten, D.L. (2012). Systematic validation of specific phenotypic markers for in vitro polarized human macrophages. J Immunol Methods 375, 196-206.

Ancuta, P., Liu, K.Y., Misra, V., Wacleche, V.S., Gosselin, A., Zhou, X., and Gabuzda, D. (2009). Transcriptional profiling reveals developmental relationship and distinct biological functions of CD16+ and CD16- monocyte subsets. BMC.Genomics 10, 403.

Andreesen, R., Brugger, W., Scheibenbogen, C., Kreutz, M., Leser, H.G., Rehm, A., and Lohr, G.W. (1990). Surface phenotype analysis of human monocyte to macrophage maturation. J Leukoc Biol 47, 490-497.

Annunziato, F., Cosmi, L., Liotta, F., Maggi, E., and Romagnani, S. (2012). Defining the human T helper 17 cell phenotype. Trends Immunol 33, 505-512.

Annunziato, F., and Romagnani, S. (2009). Heterogeneity of human effector CD4+ T cells. Arthritis Res Ther 11, 257. 
Apps, R., Murphy, S.P., Fernando, R., Gardner, L., Ahad, T., and Moffett, A. (2009). Human leucocyte antigen (HLA) expression of primary trophoblast cells and placental cell lines, determined using single antigen beads to characterize allotype specificities of anti-HLA antibodies. Immunology 127, 26-39.

Azzaoui, I., Yahia, S.A., Chang, Y., Vorng, H., Morales, O., Fan, Y., Delhem, N., Ple, C., Tonnel, A.B., Wallaert, B., and Tsicopoulos, A. (2011). CCL18 differentiates dendritic cells in tolerogenic cells able to prime regulatory $\mathrm{T}$ cells in healthy subjects. Blood 118,3549 3558 .

Baecher-Allan, C., Brown, J.A., Freeman, G.J., and Hafler, D.A. (2001). CD4+CD25high regulatory cells in human peripheral blood. J Immunol 167, 1245-1253.

Bain, C.C., Scott, C.L., Uronen-Hansson, H., Gudjonsson, S., Jansson, O., Grip, O., Guilliams, M., Malissen, B., Agace, W.W., and Mowat, A.M. (2013). Resident and proinflammatory macrophages in the colon represent alternative context-dependent fates of the same Ly6Chi monocyte precursors. Mucosal immunology 6, 498-510.

Ban, Y.L., Kong, B.H., Qu, X., Yang, Q.F., and Ma, Y.Y. (2008). BDCA-1+, BDCA-2+ and BDCA-3+ dendritic cells in early human pregnancy decidua. Clin Exp Immunol 151, 399406.

Bardel, E., Larousserie, F., Charlot-Rabiega, P., Coulomb-L'Hermine, A., and Devergne, O. (2008). Human CD4+ CD25+ Foxp3+ regulatory T cells do not constitutively express IL-35. J Immunol 181, 6898-6905.

Bartocci, A., Pollard, J.W., and Stanley, E.R. (1986). Regulation of colony-stimulating factor 1 during pregnancy. J Exp Med 164, 956-961.

Barve, R.A., Zack, M.D., Weiss, D., Song, R.H., Beidler, D., and Head, R.D. (2013). Transcriptional profiling and pathway analysis of CSF-1 and IL-34 effects on human monocyte differentiation. Cytokine 63, 10-17.

Beagley, K.W., and Gockel, C.M. (2003). Regulation of innate and adaptive immunity by the female sex hormones oestradiol and progesterone. FEMS Immunol Med Microbiol 38, 13-22.

Bellinghausen, I., Reuter, S., Martin, H., Maxeiner, J., Luxemburger, U., Tureci, O., Grabbe, S., Taube, C., and Saloga, J. (2012). Enhanced production of CCL18 by tolerogenic dendritic cells is associated with inhibition of allergic airway reactivity. J Allergy Clin Immunol 130, 1384-1393.

Benirschke, K. (1994). Anatomical relationship between fetus and mother. Ann N Y Acad Sci 731, 9-20.

Benoit, M., Desnues, B., and Mege, J.L. (2008). Macrophage polarization in bacterial infections. J Immunol 181, 3733-3739.

Biswas, S.K., and Mantovani, A. (2010). Macrophage plasticity and interaction with lymphocyte subsets: cancer as a paradigm. Nat Immunol 11, 889-896.

Biswas, S.K., and Mantovani, A. (2012). Orchestration of metabolism by macrophages. Cell metabolism 15, 432-437. 
Blanchard, C., Wang, N., Stringer, K.F., Mishra, A., Fulkerson, P.C., Abonia, J.P., Jameson, S.C., Kirby, C., Konikoff, M.R., Collins, M.H., Cohen, M.B., Akers, R., Hogan, S.P., Assa'ad, A.H., Putnam, P.E., Aronow, B.J., and Rothenberg, M.E. (2006). Eotaxin-3 and a uniquely conserved gene-expression profile in eosinophilic esophagitis. J Clin Invest 116, 536-547.

Blois, S.M., Ilarregui, J.M., Tometten, M., Garcia, M., Orsal, A.S., Cordo-Russo, R., Toscano, M.A., Bianco, G.A., Kobelt, P., Handjiski, B., Tirado, I., Markert, U.R., Klapp, B.F., Poirier, F., Szekeres-Bartho, J., Rabinovich, G.A., and Arck, P.C. (2007). A pivotal role for galectin-1 in fetomaternal tolerance. Nat Med 13, 1450-1457.

Bockle, B.C., Solder, E., Kind, S., Romani, N., and Sepp, N.T. (2008). DC-SIGN+ CD163+ Macrophages Expressing Hyaluronan Receptor LYVE-1 Are Located within Chorion Villi of the Placenta. Placenta 29, 187-192.

Boij, R., Svensson, J., Nilsson-Ekdahl, K., Sandholm, K., Lindahl, T.L., Palonek, E., Garle, M., Berg, G., Ernerudh, J., Jenmalm, M., and Matthiesen, L. (2012). Biomarkers of Coagulation, Inflammation, and Angiogenesis are Independently Associated with Preeclampsia. Am J Reprod Immunol 68, 258-270.

Borsellino, G., Kleinewietfeld, M., Di Mitri, D., Sternjak, A., Diamantini, A., Giometto, R., Hopner, S., Centonze, D., Bernardi, G., Dell'Acqua, M.L., Rossini, P.M., Battistini, L., Rotzschke, O., and Falk, K. (2007). Expression of ectonucleotidase CD39 by Foxp3+ Treg cells: hydrolysis of extracellular ATP and immune suppression. Blood 110, 1225-1232.

Borzychowski, A.M., Croy, B.A., Chan, W.L., Redman, C.W., and Sargent, I.L. (2005). Changes in systemic type 1 and type 2 immunity in normal pregnancy and pre-eclampsia may be mediated by natural killer cells. Eur.J.Immunol. 35, 3054-3063.

Brocheriou, I., Maouche, S., Durand, H., Braunersreuther, V., Le Naour, G., Gratchev, A., Koskas, F., Mach, F., Kzhyshkowska, J., and Ninio, E. (2011). Antagonistic regulation of macrophage phenotype by M-CSF and GM-CSF: implication in atherosclerosis. Atherosclerosis 214, 316-324.

Bruder, D., Probst-Kepper, M., Westendorf, A.M., Geffers, R., Beissert, S., Loser, K., von Boehmer, H., Buer, J., and Hansen, W. (2004). Neuropilin-1: a surface marker of regulatory T cells. Eur J Immunol 34, 623-630.

Brugger, W., Kreutz, M., and Andreesen, R. (1991). Macrophage colony-stimulating factor is required for human monocyte survival and acts as a cofactor for their terminal differentiation to macrophages in vitro. J Leukoc Biol 49, 483-488.

Buechler, C., Ritter, M., Orso, E., Langmann, T., Klucken, J., and Schmitz, G. (2000). Regulation of scavenger receptor CD163 expression in human monocytes and macrophages by pro- and antiinflammatory stimuli. J Leukoc Biol 67, 97-103.

Bulmer, J.N., and Johnson, P.M. (1984). Macrophage populations in the human placenta and amniochorion. Clin Exp Immunol 57, 393-403.

Burk, M.R., Troeger, C., Brinkhaus, R., Holzgreve, W., and Hahn, S. (2001). Severely reduced presence of tissue macrophages in the basal plate of pre-eclamptic placentae. Placenta 22, 309-316. 
Canavan, J.B., Afzali, B., Scotta, C., Fazekasova, H., Edozie, F.C., Macdonald, T.T., Hernandez-Fuentes, M.P., Lombardi, G., and Lord, G.M. (2012). A rapid diagnostic test for human regulatory T-cell function to enable regulatory T-cell therapy. Blood 119, e57-66.

Carlino, C., Stabile, H., Morrone, S., Bulla, R., Soriani, A., Agostinis, C., Bossi, F., Mocci, C., Sarazani, F., Tedesco, F., Santoni, A., and Gismondi, A. (2008). Recruitment of circulating NK cells through decidual tissues: a possible mechanism controlling NK cell accumulation in the uterus during early pregnancy. Blood 111,3108-3115.

Cartwright, J.E., Fraser, R., Leslie, K., Wallace, A.E., and James, J.L. (2010). Remodelling at the maternal-fetal interface: relevance to human pregnancy disorders. Reproduction 140, 803813.

Chang, C.J., Yen, M.L., Chen, Y.C., Chien, C.C., Huang, H.I., Bai, C.H., and Yen, B.L. (2006). Placenta-derived multipotent cells exhibit immunosuppressive properties that are enhanced in the presence of interferon-gamma. Stem cells 24, 2466-2477.

Chang, H.H., Larson, J., Blencowe, H., Spong, C.Y., Howson, C.P., Cairns-Smith, S., Lackritz, E.M., Lee, S.K., Mason, E., Serazin, A.C., Walani, S., Simpson, J.L., Lawn, J.E., and Born Too Soon preterm prevention analysis, g. (2013). Preventing preterm births: analysis of trends and potential reductions with interventions in 39 countries with very high human development index. Lancet 381, 223-234.

Chang, Y., de Nadai, P., Azzaoui, I., Morales, O., Delhem, N., Vorng, H., Tomavo, S., Ait Yahia, S., Zhang, G., Wallaert, B., Chenivesse, C., and Tsicopoulos, A. (2010). The chemokine CCL18 generates adaptive regulatory T cells from memory CD4+ T cells of healthy but not allergic subjects. FASEB J 24, 5063-5072.

Chaouat, G., Assal Meliani, A., Martal, J., Raghupathy, R., Elliott, J.F., Mosmann, T., and Wegmann, T.G. (1995). IL-10 prevents naturally occurring fetal loss in the CBA x DBA/2 mating combination, and local defect in IL-10 production in this abortion-prone combination is corrected by in vivo injection of IFN-tau. J Immunol 154, 4261-4268.

Chaouat, G., Cayol, V., Mairovitz, V., and Dubanchet, S. (1999). Localization of the Th2 cytokines IL-3, IL-4, IL-10 at the fetomaternal interface during human and murine pregnancy and lack of requirement for Fas/Fas ligand interaction for a successful allogeneic pregnancy. Am J Reprod Immunol 42, 1-13.

Chaturvedi, V., Ertelt, J.M., Jiang, T.T., Kinder, J.M., Xin, L., Owens, K.J., Jones, H.N., and Way, S.S. (2015). CXCR3 blockade protects against Listeria monocytogenes infectioninduced fetal wastage. J Clin Invest 125, 1713-1725.

Chen, W., Jin, W., Hardegen, N., Lei, K.J., Li, L., Marinos, N., McGrady, G., and Wahl, S.M. (2003). Conversion of peripheral CD4+CD25- naive T cells to CD4+CD25+ regulatory $\mathrm{T}$ cells by TGF-beta induction of transcription factor Foxp3. J Exp Med 198, 1875-1886.

Chenivesse, C., Chang, Y., Azzaoui, I., Ait Yahia, S., Morales, O., Ple, C., Foussat, A., Tonnel, A.B., Delhem, N., Yssel, H., Vorng, H., Wallaert, B., and Tsicopoulos, A. (2012). Pulmonary CCL18 recruits human regulatory T cells. J Immunol 189, 128-137.

Chieppa, M., Bianchi, G., Doni, A., Del Prete, A., Sironi, M., Laskarin, G., Monti, P., Piemonti, L., Biondi, A., Mantovani, A., Introna, M., and Allavena, P. (2003). Cross-linking 
of the mannose receptor on monocyte-derived dendritic cells activates an anti-inflammatory immunosuppressive program. J Immunol 171, 4552-4560.

Co, E.C., Gormley, M., Kapidzic, M., Rosen, D.B., Scott, M.A., Stolp, H.A., McMaster, M., Lanier, L.L., Barcena, A., and Fisher, S.J. (2013). Maternal decidual macrophages inhibit NK cell killing of invasive cytotrophoblasts during human pregnancy. Biol Reprod 88, 155.

Codarri, L., Gyulveszi, G., Tosevski, V., Hesske, L., Fontana, A., Magnenat, L., Suter, T., and Becher, B. (2011). RORgammat drives production of the cytokine GM-CSF in helper T cells, which is essential for the effector phase of autoimmune neuroinflammation. Nat Immunol 12, 560-567.

Collins, M.K., Tay, C.S., and Erlebacher, A. (2009). Dendritic cell entrapment within the pregnant uterus inhibits immune surveillance of the maternal/fetal interface in mice. J Clin Invest 119, 2062-2073.

Collison, L.W., Chaturvedi, V., Henderson, A.L., Giacomin, P.R., Guy, C., Bankoti, J., Finkelstein, D., Forbes, K., Workman, C.J., Brown, S.A., Rehg, J.E., Jones, M.L., Ni, H.T., Artis, D., Turk, M.J., and Vignali, D.A. (2010). IL-35-mediated induction of a potent regulatory T cell population. Nat Immunol 11, 1093-1101.

Collison, L.W., Workman, C.J., Kuo, T.T., Boyd, K., Wang, Y., Vignali, K.M., Cross, R., Sehy, D., Blumberg, R.S., and Vignali, D.A. (2007). The inhibitory cytokine IL-35 contributes to regulatory T-cell function. Nature 450, 566-569.

Confavreux, C., Hutchinson, M., Hours, M.M., Cortinovis-Tourniaire, P., and Moreau, T. (1998). Rate of pregnancy-related relapse in multiple sclerosis. Pregnancy in Multiple Sclerosis Group. N.Engl.J.Med. 339, 285-291.

Conti, L., and Gessani, S. (2008). GM-CSF in the generation of dendritic cells from human blood monocyte precursors: Recent advances. Immunobiology 213, 859-870.

Cooper, M.A., Fehniger, T.A., and Caligiuri, M.A. (2001). The biology of human natural killer-cell subsets. Trends Immunol 22, 633-640.

Cordeau, M., Herblot, S., Charrier, E., Audibert, F., Cordeiro, P., Harnois, M., and Duval, M. (2012). Defects in CD54 and CD86 up-regulation by plasmacytoid dendritic cells during pregnancy. Immunol.Invest 41, 497-506.

Cros, J., Cagnard, N., Woollard, K., Patey, N., Zhang, S.Y., Senechal, B., Puel, A., Biswas, S.K., Moshous, D., Picard, C., Jais, J.P., D'Cruz, D., Casanova, J.L., Trouillet, C., and Geissmann, F. (2010). Human CD14dim monocytes patrol and sense nucleic acids and viruses via TLR7 and TLR8 receptors. Immunity 33, 375-386.

Darmochwal-Kolarz, D.A., Kludka-Sternik, M., Chmielewski, T., Kolarz, B., Rolinski, J., Leszczynska-Gorzelak, B., and Oleszczuk, J. (2012). The expressions of CD200 and CD200R molecules on myeloid and lymphoid dendritic cells in pre-eclampsia and normal pregnancy. American Journal of Reproductive Immunology 67, 474-481.

Davies, L.C., Jenkins, S.J., Allen, J.E., and Taylor, P.R. (2013). Tissue-resident macrophages. Nat Immunol 14, 986-995. 
De Oliveira, L.G., Lash, G.E., Murray-Dunning, C., Bulmer, J.N., Innes, B.A., Searle, R.F., Sass, N., and Robson, S.C. (2010). Role of interleukin 8 in uterine natural killer cell regulation of extravillous trophoblast cell invasion. Placenta 31, 595-601.

Devergne, O., Birkenbach, M., and Kieff, E. (1997). Epstein-Barr virus-induced gene 3 and the p35 subunit of interleukin 12 form a novel heterodimeric hematopoietin. Proc Natl Acad Sci U S A 94, 12041-12046.

Dimova, T., Nagaeva, O., Stenqvist, A.C., Hedlund, M., Kjellberg, L., Strand, M., Dehlin, E., and Mincheva-Nilsson, L. (2011). Maternal Foxp3 expressing CD4+ CD25+ and CD4+ CD25- regulatory T-cell populations are enriched in human early normal pregnancy decidua: a phenotypic study of paired decidual and peripheral blood samples. Am J Reprod Immunol 66 Suppl 1, 44-56.

Doherty, T.A. (2015). At the Bench: Understanding group 2 innate lymphoid cells in disease. J Leukoc Biol 97, 455-467.

Dominguez-Soto, A., Sierra-Filardi, E., Puig-Kroger, A., Perez-Maceda, B., Gomez-Aguado, F., Corcuera, M.T., Sanchez-Mateos, P., and Corbi, A.L. (2011). Dendritic cell-specific ICAM-3-grabbing nonintegrin expression on M2-polarized and tumor-associated macrophages is macrophage-CSF dependent and enhanced by tumor-derived IL-6 and IL-10. J Immunol 186, 2192-2200.

Drake, P.M., Gunn, M.D., Charo, I.F., Tsou, C.L., Zhou, Y., Huang, L., and Fisher, S.J. (2001). Human placental cytotrophoblasts attract monocytes and CD56(bright) natural killer cells via the actions of monocyte inflammatory protein lalpha. J Exp Med 193, 1199-1212.

Du, M.R., Guo, P.F., Piao, H.L., Wang, S.C., Sun, C., Jin, L.P., Tao, Y., Li, Y.H., Zhang, D., Zhu, R., Fu, Q., and Li, D.J. (2014a). Embryonic Trophoblasts Induce Decidual Regulatory T Cell Differentiation and Maternal-Fetal Tolerance through Thymic Stromal Lymphopoietin Instructing Dendritic Cells. J Immunol 192, 1502-1511.

Du, M.R., Wang, S.C., and Li, D.J. (2014b). The integrative roles of chemokines at the maternal-fetal interface in early pregnancy. Cellular \& molecular immunology 11, 438-448.

Duhen, T., Duhen, R., Lanzavecchia, A., Sallusto, F., and Campbell, D.J. (2012).

Functionally distinct subsets of human FOXP3+ Treg cells that phenotypically mirror effector Th cells. Blood 119, 4430-4440.

Duriez, M., Quillay, H., Madec, Y., El Costa, H., Cannou, C., Marlin, R., de Truchis, C., Rahmati, M., Barre-Sinoussi, F., Nugeyre, M.T., and Menu, E. (2014). Human decidual macrophages and NK cells differentially express Toll-like receptors and display distinct cytokine profiles upon TLR stimulation. Frontiers in microbiology 5, 316.

Engert, S., Rieger, L., Kapp, M., Becker, J.C., Dietl, J., and Kammerer, U. (2007). Profiling chemokines, cytokines and growth factors in human early pregnancy decidua by protein array. Am J Reprod Immunol 58, 129-137.

Erkers, T., Nava, S., Yosef, J., Ringden, O., and Kaipe, H. (2013). Decidual stromal cells promote regulatory $\mathrm{T}$ cells and suppress alloreactivity in a cell contact-dependent manner. Stem Cells Dev 22, 2596-2605. 
Erlebacher, A., Vencato, D., Price, K.A., Zhang, D., and Glimcher, L.H. (2007). Constraints in antigen presentation severely restrict $\mathrm{T}$ cell recognition of the allogeneic fetus. J Clin Invest 117, 1399-1411.

Ernerudh, J., Berg, G., and Mjosberg, J. (2011). Regulatory T helper cells in pregnancy and their roles in systemic versus local immune tolerance. Am J Reprod Immunol 66 Suppl 1, 31 43.

Estecha, A., Aguilera-Montilla, N., Sanchez-Mateos, P., and Puig-Kroger, A. (2012). RUNX3 regulates intercellular adhesion molecule 3 (ICAM-3) expression during macrophage differentiation and monocyte extravasation. PLoS One 7, e33313.

Fabriek, B.O., Dijkstra, C.D., and van den Berg, T.K. (2005). The macrophage scavenger receptor CD163. Immunobiology 210, 153-160.

Fabriek, B.O., van Bruggen, R., Deng, D.M., Ligtenberg, A.J., Nazmi, K., Schornagel, K., Vloet, R.P., Dijkstra, C.D., and van den Berg, T.K. (2009). The macrophage scavenger receptor CD163 functions as an innate immune sensor for bacteria. Blood 113, 887-892.

Ferber, I.A., Lee, H.J., Zonin, F., Heath, V., Mui, A., Arai, N., and O'Garra, A. (1999). GATA-3 significantly downregulates IFN-gamma production from developing Th1 cells in addition to inducing IL-4 and IL-5 levels. Clin Immunol 91, 134-144.

Fleetwood, A.J., Lawrence, T., Hamilton, J.A., and Cook, A.D. (2007). Granulocytemacrophage colony-stimulating factor (CSF) and macrophage CSF-dependent macrophage phenotypes display differences in cytokine profiles and transcription factor activities: implications for CSF blockade in inflammation. J Immunol 178, 5245-5252.

Fock, V., Mairhofer, M., Otti, G.R., Hiden, U., Spittler, A., Zeisler, H., Fiala, C., Knofler, M., and Pollheimer, J. (2013). Macrophage-derived IL-33 is a critical factor for placental growth. J Immunol 191, 3734-3743.

Fontenot, J.D., Gavin, M.A., and Rudensky, A.Y. (2003). Foxp3 programs the development and function of CD4+CD25+ regulatory T cells. Nat Immunol 4, 330-336.

Foucher, E.D., Blanchard, S., Preisser, L., Garo, E., Ifrah, N., Guardiola, P., Delneste, Y., and Jeannin, P. (2013). IL-34 induces the differentiation of human monocytes into immunosuppressive macrophages. antagonistic effects of GM-CSF and IFNgamma. PLoS One 8 , e56045.

Fraser, R., Whitley, G.S., Johnstone, A.P., Host, A.J., Sebire, N.J., Thilaganathan, B., and Cartwright, J.E. (2012). Impaired decidual natural killer cell regulation of vascular remodelling in early human pregnancies with high uterine artery resistance. J Pathol 228, $322-332$.

Gaffen, S.L., Jain, R., Garg, A.V., and Cua, D.J. (2014). The IL-23-IL-17 immune axis: from mechanisms to therapeutic testing. Nat Rev Immunol 14, 585-600.

Gao, W., Thompson, L., Zhou, Q., Putheti, P., Fahmy, T.M., Strom, T.B., and Metcalfe, S.M. (2009). Treg versus Th17 lymphocyte lineages are cross-regulated by LIF versus IL-6. Cell Cycle 8, 1444-1450. 
Gardner, L., and Moffett, A. (2003). Dendritic cells in the human decidua. Biol Reprod 69, 1438-1446.

Geijtenbeek, T.B., and Gringhuis, S.I. (2009). Signalling through C-type lectin receptors: shaping immune responses. Nat Rev Immunol 9, 465-479.

Geissmann, F., Manz, M.G., Jung, S., Sieweke, M.H., Merad, M., and Ley, K. (2010). Development of monocytes, macrophages, and dendritic cells. Science 327, 656-661.

Germain, S.J., Sacks, G.P., Sooranna, S.R., Sargent, I.L., and Redman, C.W. (2007). Systemic inflammatory priming in normal pregnancy and preeclampsia: the role of circulating syncytiotrophoblast microparticles. J Immunol 178, 5949-5956.

Ginhoux, F., Greter, M., Leboeuf, M., Nandi, S., See, P., Gokhan, S., Mehler, M.F., Conway, S.J., Ng, L.G., Stanley, E.R., Samokhvalov, I.M., and Merad, M. (2010). Fate mapping analysis reveals that adult microglia derive from primitive macrophages. Science 330, 841845.

Ginhoux, F., and Jung, S. (2014). Monocytes and macrophages: developmental pathways and tissue homeostasis. Nat Rev Immunol 14, 392-404.

Gohner, C., Svensson-Arvelund, J., Pfarrer, C., Hager, J.D., Faas, M., Ernerudh, J., Cline, J.M., Dixon, D., Buse, E., and Markert, U.R. (2014). The placenta in toxicology. Part IV: Battery of toxicological test systems based on human placenta. Toxicologic pathology 42 , 345-351.

Gordon, S. (2003). Alternative activation of macrophages. Nat Rev Immunol 3, 23-35.

Gordon, S., Pluddemann, A., and Martinez Estrada, F. (2014). Macrophage heterogeneity in tissues: phenotypic diversity and functions. Immunol Rev 262, 36-55.

Goriely, S., and Goldman, M. (2008). Interleukin-12 family members and the balance between rejection and tolerance. Current opinion in organ transplantation 13, 4-9.

Gotsch, F., Romero, R., Friel, L., Kusanovic, J.P., Espinoza, J., Erez, O., Than, N.G., Mittal, P., Edwin, S., Yoon, B.H., Kim, C.J., Mazaki-Tovi, S., Chaiworapongsa, T., and Hassan, S.S. (2007). CXCL10/IP-10: a missing link between inflammation and anti-angiogenesis in preeclampsia? J Matern Fetal Neonatal Med 20, 777-792.

Graham, C.H., Hawley, T.S., Hawley, R.G., MacDougall, J.R., Kerbel, R.S., Khoo, N., and Lala, P.K. (1993). Establishment and characterization of first trimester human trophoblast cells with extended lifespan. Exp Cell Res 206, 204-211.

Grange, C., Letourneau, J., Forget, M.A., Godin-Ethier, J., Martin, J., Liberman, M., Latour, M., Widmer, H., Lattouf, J.B., Piccirillo, C.A., Cailhier, J.F., and Lapointe, R. (2011). Phenotypic characterization and functional analysis of human tumor immune infiltration after mechanical and enzymatic disaggregation. J Immunol Methods 372, 119-126.

Gratacos, E., Filella, X., Palacio, M., Cararach, V., Alonso, P.L., and Fortuny, A. (1998). Interleukin-4, interleukin-10, and granulocyte-macrophage colony stimulating factor in second-trimester serum from women with preeclampsia. Obstet Gynecol 92, 849-853. 
Greer, I.A., Lyall, F., Perera, T., Boswell, F., and Macara, L.M. (1994). Increased concentrations of cytokines interleukin- 6 and interleukin-1 receptor antagonist in plasma of women with preeclampsia: a mechanism for endothelial dysfunction? Obstet Gynecol 84, 937-940.

Greter, M., Helft, J., Chow, A., Hashimoto, D., Mortha, A., Agudo-Cantero, J., Bogunovic, M., Gautier, E.L., Miller, J., Leboeuf, M., Lu, G., Aloman, C., Brown, B.D., Pollard, J.W., Xiong, H., Randolph, G.J., Chipuk, J.E., Frenette, P.S., and Merad, M. (2012a). GM-CSF controls nonlymphoid tissue dendritic cell homeostasis but is dispensable for the differentiation of inflammatory dendritic cells. Immunity 36, 1031-1046.

Greter, M., Lelios, I., Pelczar, P., Hoeffel, G., Price, J., Leboeuf, M., Kundig, T.M., Frei, K., Ginhoux, F., Merad, M., and Becher, B. (2012b). Stroma-derived interleukin-34 controls the development and maintenance of langerhans cells and the maintenance of microglia. Immunity 37, 1050-1060.

Griffith, J.W., Sokol, C.L., and Luster, A.D. (2014). Chemokines and chemokine receptors: positioning cells for host defense and immunity. Annu Rev Immunol 32, 659-702.

Groux, H., Bigler, M., de Vries, J.E., and Roncarolo, M.G. (1996). Interleukin-10 induces a long-term antigen-specific anergic state in human CD4+ T cells. J Exp Med 184, 19-29.

Gude, N.M., Roberts, C.T., Kalionis, B., and King, R.G. (2004). Growth and function of the normal human placenta. Thrombosis research 114, 397-407.

Guerin, L.R., Prins, J.R., and Robertson, S.A. (2009). Regulatory T-cells and immune tolerance in pregnancy: a new target for infertility treatment? Hum Reprod Update 15, 517535.

Gustafsson, C., Mjosberg, J., Matussek, A., Geffers, R., Matthiesen, L., Berg, G., Sharma, S., Buer, J., and Ernerudh, J. (2008). Gene expression profiling of human decidual macrophages: evidence for immunosuppressive phenotype. PLoS ONE 3, e2078.

Hamilton, J.A. (2008). Colony-stimulating factors in inflammation and autoimmunity. Nat Rev Immunol 8, 533-544.

Hamilton, J.A., and Achuthan, A. (2013). Colony stimulating factors and myeloid cell biology in health and disease. Trends Immunol 34, 81-89.

Hamilton, S.A., Tower, C.L., and Jones, R.L. (2013). Identification of chemokines associated with the recruitment of decidual leukocytes in human labour: potential novel targets for preterm labour. PLoS One 8, e56946.

Hanna, J., Goldman-Wohl, D., Hamani, Y., Avraham, I., Greenfield, C., Natanson-Yaron, S., Prus, D., Cohen-Daniel, L., Arnon, T.I., Manaster, I., Gazit, R., Yutkin, V., Benharroch, D., Porgador, A., Keshet, E., Yagel, S., and Mandelboim, O. (2006). Decidual NK cells regulate key developmental processes at the human fetal-maternal interface. Nat Med 12, 1065-1074.

Hanna, J., Wald, O., Goldman-Wohl, D., Prus, D., Markel, G., Gazit, R., Katz, G., HaimovKochman, R., Fujii, N., Yagel, S., Peled, A., and Mandelboim, O. (2003). CXCL12 expression by invasive trophoblasts induces the specific migration of CD16- human natural killer cells. Blood 102, 1569-1577. 
Hanna, N., Hanna, I., Hleb, M., Wagner, E., Dougherty, J., Balkundi, D., Padbury, J., and Sharma, S. (2000). Gestational age-dependent expression of IL-10 and its receptor in human placental tissues and isolated cytotrophoblasts. J Immunol 164, 5721-5728.

Harrington, L.E., Hatton, R.D., Mangan, P.R., Turner, H., Murphy, T.L., Murphy, K.M., and Weaver, C.T. (2005). Interleukin 17-producing CD4+ effector T cells develop via a lineage distinct from the T helper type 1 and 2 lineages. Nat Immunol 6, 1123-1132.

Hashimoto, D., Chow, A., Noizat, C., Teo, P., Beasley, M.B., Leboeuf, M., Becker, C.D., See, P., Price, J., Lucas, D., Greter, M., Mortha, A., Boyer, S.W., Forsberg, E.C., Tanaka, M., van Rooijen, N., Garcia-Sastre, A., Stanley, E.R., Ginhoux, F., Frenette, P.S., and Merad, M. (2013). Tissue-resident macrophages self-maintain locally throughout adult life with minimal contribution from circulating monocytes. Immunity 38, 792-804.

Hatayama, H., Kanzaki, H., Iwai, M., Kariya, M., Fujimoto, M., Higuchi, T., Kojima, K., Nakayama, H., Mori, T., and Fujita, J. (1994). Progesterone enhances macrophage colonystimulating factor production in human endometrial stromal cells in vitro. Endocrinology 135, 1921-1927.

Hayashi, M., Hamada, Y., and Ohkura, T. (2004). Elevation of granulocyte-macrophage colony-stimulating factor in the placenta and blood in preeclampsia. Am J Obstet Gynecol 190, 456-461.

Hayashida, K., Nanki, T., Girschick, H., Yavuz, S., Ochi, T., and Lipsky, P.E. (2001). Synovial stromal cells from rheumatoid arthritis patients attract monocytes by producing MCP-1 and IL-8. Arthritis research 3, 118-126.

Haynes, D.R., Whitehouse, M.W., and Vernon-Roberts, B. (1992). The prostaglandin E1 analogue, misoprostol, regulates inflammatory cytokines and immune functions in vitro like the natural prostaglandins E1, E2 and E3. Immunology 76, 251-257.

Hazan, A.D., Smith, S.D., Jones, R.L., Whittle, W., Lye, S.J., and Dunk, C.E. (2010). Vascular-leukocyte interactions: mechanisms of human decidual spiral artery remodeling in vitro. Am J Pathol 177, 1017-1030.

He, Y.Y., He, X.J., Guo, P.F., Du, M.R., Shao, J., Li, M.Q., and Li, D.J. (2012). The decidual stromal cells-secreted CCL2 induces and maintains decidual leukocytes into Th2 bias in human early pregnancy. Clin Immunol 145, 161-173.

Heikkinen, J., Mottonen, M., Alanen, A., and Lassila, O. (2004). Phenotypic characterization of regulatory T cells in the human decidua. Clin Exp Immunol 136, 373-378.

Heikkinen, J., Mottonen, M., Komi, J., Alanen, A., and Lassila, O. (2003). Phenotypic characterization of human decidual macrophages. Clin Exp Immunol 131, 498-505.

Helige, C., Ahammer, H., Moser, G., Hammer, A., Dohr, G., Huppertz, B., and Sedlmayr, P. (2014). Distribution of decidual natural killer cells and macrophages in the neighbourhood of the trophoblast invasion front: a quantitative evaluation. Hum Reprod 29, 8-17.

Hennessy, A., Pilmore, H.L., Simmons, L.A., and Painter, D.M. (1999). A deficiency of placental IL-10 in preeclampsia. J Immunol 163, 3491-3495. 
Hiby, S.E., Walker, J.J., O'Shaughnessy K, M., Redman, C.W., Carrington, M., Trowsdale, J., and Moffett, A. (2004). Combinations of maternal KIR and fetal HLA-C genes influence the risk of preeclampsia and reproductive success. J Exp Med 200, 957-965.

Hoeffel, G., Wang, Y., Greter, M., See, P., Teo, P., Malleret, B., Leboeuf, M., Low, D., Oller, G., Almeida, F., Choy, S.H., Grisotto, M., Renia, L., Conway, S.J., Stanley, E.R., Chan, J.K., Ng, L.G., Samokhvalov, I.M., Merad, M., and Ginhoux, F. (2012). Adult Langerhans cells derive predominantly from embryonic fetal liver monocytes with a minor contribution of yolk sac-derived macrophages. J Exp Med 209, 1167-1181.

Houser, B.L., Tilburgs, T., Hill, J., Nicotra, M.L., and Strominger, J.L. (2011). Two unique human decidual macrophage populations. J Immunol 186, 2633-2642.

Hsieh, C.S., Macatonia, S.E., Tripp, C.S., Wolf, S.F., O'Garra, A., and Murphy, K.M. (1993). Development of TH1 CD4+ T cells through IL-12 produced by Listeria-induced macrophages. Science 260, 547-549.

Hsu, P., Santner-Nanan, B., Dahlstrom, J.E., Fadia, M., Chandra, A., Peek, M., and Nanan, R. (2012). Altered decidual DC-SIGN+ antigen-presenting cells and impaired regulatory T-cell induction in preeclampsia. Am J Pathol 181, 2149-2160.

Huang, S.J., Zenclussen, A.C., Chen, C.P., Basar, M., Yang, H., Arcuri, F., Li, M., Kocamaz, E., Buchwalder, L., Rahman, M., Kayisli, U., Schatz, F., Toti, P., and Lockwood, C.J. (2010). The Implication of Aberrant GM-CSF Expression in Decidual Cells in the Pathogenesis of Preeclampsia. Am J Pathol 177, 2472-2482.

Huang, Y., Zhu, X.Y., Du, M.R., and Li, D.J. (2008). Human trophoblasts recruited T lymphocytes and monocytes into decidua by secretion of chemokine CXCL16 and interaction with CXCR6 in the first-trimester pregnancy. J Immunol 180, 2367-2375.

Hume, D.A., and MacDonald, K.P. (2012). Therapeutic applications of macrophage colonystimulating factor-1 (CSF-1) and antagonists of CSF-1 receptor (CSF-1R) signaling. Blood $119,1810-1820$.

Hunt, J.S., Petroff, M.G., McIntire, R.H., and Ober, C. (2005). HLA-G and immune tolerance in pregnancy. FASEB J 19, 681-693.

Hunt, J.S., Vassmer, D., Ferguson, T.A., and Miller, L. (1997). Fas ligand is positioned in mouse uterus and placenta to prevent trafficking of activated leukocytes between the mother and the conceptus. J Immunol 158, 4122-4128.

Hwang, S.J., Choi, B., Kang, S.S., Chang, J.H., Kim, Y.G., Chung, Y.H., Sohn, D.H., So, M.W., Lee, C.K., Robinson, W.H., and Chang, E.J. (2012). Interleukin-34 produced by human fibroblast-like synovial cells in rheumatoid arthritis supports osteoclastogenesis. Arthritis Res Ther 14, R14.

Iellem, A., Mariani, M., Lang, R., Recalde, H., Panina-Bordignon, P., Sinigaglia, F., and D'Ambrosio, D. (2001). Unique chemotactic response profile and specific expression of chemokine receptors CCR 4 and CCR 8 by CD4(+)CD25(+) regulatory T cells. J Exp Med 194, 847-853. 
Ikeda, T., Hirata, S., Fukushima, S., Matsunaga, Y., Ito, T., Uchino, M., Nishimura, Y., and Senju, S. (2010). Dual effects of TRAIL in suppression of autoimmunity: the inhibition of Th1 cells and the promotion of regulatory T cells. J Immunol 185, 5259-5267.

Islam, S.A., Ling, M.F., Leung, J., Shreffler, W.G., and Luster, A.D. (2013). Identification of human CCR8 as a CCL18 receptor. J Exp Med 210, 1889-1898.

Islam, S.A., and Luster, A.D. (2012). T cell homing to epithelial barriers in allergic disease. Nat Med 18, 705-715.

Ivanov, II, McKenzie, B.S., Zhou, L., Tadokoro, C.E., Lepelley, A., Lafaille, J.J., Cua, D.J., and Littman, D.R. (2006). The orphan nuclear receptor RORgammat directs the differentiation program of proinflammatory IL-17+ T helper cells. Cell 126, 1121-1133.

Jaguin, M., Houlbert, N., Fardel, O., and Lecureur, V. (2013). Polarization profiles of human M-CSF-generated macrophages and comparison of M1-markers in classically activated macrophages from GM-CSF and M-CSF origin. Cellular immunology 281, 51-61.

Jenkins, S.J., Ruckerl, D., Cook, P.C., Jones, L.H., Finkelman, F.D., van Rooijen, N., MacDonald, A.S., and Allen, J.E. (2011). Local macrophage proliferation, rather than recruitment from the blood, is a signature of TH2 inflammation. Science 332, 1284-1288.

Ji, J.D., Park-Min, K.H., and Ivashkiv, L.B. (2009). Expression and function of semaphorin $3 \mathrm{~A}$ and its receptors in human monocyte-derived macrophages. Hum Immunol 70, 211-217.

Jiang, T.T., Chaturvedi, V., Ertelt, J.M., Kinder, J.M., Clark, D.R., Valent, A.M., Xin, L., and Way, S.S. (2014). Regulatory T cells: new keys for further unlocking the enigma of fetal tolerance and pregnancy complications. J Immunol 192, 4949-4956.

Joerink, M., Rindsjo, E., van Riel, B., Alm, J., and Papadogiannakis, N. (2011). Placental macrophage (Hofbauer cell) polarization is independent of maternal allergen-sensitization and presence of chorioamnionitis. Placenta 32, 380-385.

Jokhi, P.P., King, A., and Loke, Y.W. (1994). Production of granulocyte-macrophage colonystimulating factor by human trophoblast cells and by decidual large granular lymphocytes. Hum Reprod 9, 1660-1669.

Jones, L.A., Anthony, J.P., Henriquez, F.L., Lyons, R.E., Nickdel, M.B., Carter, K.C., Alexander, J., and Roberts, C.W. (2008). Toll-like receptor-4-mediated macrophage activation is differentially regulated by progesterone via the glucocorticoid and progesterone receptors. Immunology 125, 59-69.

Jude, C., Dejica, D., Samasca, G., Balacescu, L., and Balacescu, O. (2013). Soluble CD163 serum levels are elevated and correlated with IL-12 and CXCL10 in patients with longstanding rheumatoid arthritis. Rheumatology international 33, 1031-1037.

Kalkunte, S.S., Mselle, T.F., Norris, W.E., Wira, C.R., Sentman, C.L., and Sharma, S. (2009). Vascular endothelial growth factor $\mathrm{C}$ facilitates immune tolerance and endovascular activity of human uterine NK cells at the maternal-fetal interface. J Immunol 182, 4085-4092. 
Kallikourdis, M., Andersen, K.G., Welch, K.A., and Betz, A.G. (2007). Alloantigenenhanced accumulation of CCR5+ 'effector' regulatory T cells in the gravid uterus. Proc Natl Acad Sci U S A 104, 594-599.

Kammerer, U., Eggert, A.O., Kapp, M., McLellan, A.D., Geijtenbeek, T.B., Dietl, J., van Kooyk, Y., and Kampgen, E. (2003). Unique appearance of proliferating antigen-presenting cells expressing DC-SIGN (CD209) in the decidua of early human pregnancy. Am J Pathol $162,887-896$.

Keskin, D.B., Allan, D.S., Rybalov, B., Andzelm, M.M., Stern, J.N., Kopcow, H.D., Koopman, L.A., and Strominger, J.L. (2007). TGFbeta promotes conversion of CD16+ peripheral blood NK cells into CD16- NK cells with similarities to decidual NK cells. Proc Natl Acad Sci U S A 104, 3378-3383.

Khil, L.Y., Jun, H.S., Kwon, H., Yoo, J.K., Kim, S., Notkins, A.L., and Yoon, J.W. (2007). Human chorionic gonadotropin is an immune modulator and can prevent autoimmune diabetes in NOD mice. Diabetologia 50, 2147-2155.

Kho, E.M., McCowan, L.M., North, R.A., Roberts, C.T., Chan, E., Black, M.A., Taylor, R.S., and Dekker, G.A. (2009). Duration of sexual relationship and its effect on preeclampsia and small for gestational age perinatal outcome. J Reprod Immunol 82, 66-73.

Kim, J.S., Romero, R., Cushenberry, E., Kim, Y.M., Erez, O., Nien, J.K., Yoon, B.H., Espinoza, J., and Kim, C.J. (2007). Distribution of CD14+ and CD68+ macrophages in the placental bed and basal plate of women with preeclampsia and preterm labor. Placenta 28 , 571-576.

Kim, S.Y., Romero, R., Tarca, A.L., Bhatti, G., Kim, C.J., Lee, J., Elsey, A., Than, N.G., Chaiworapongsa, T., Hassan, S.S., Kang, G.H., and Kim, J.S. (2012). Methylome of fetal and maternal monocytes and macrophages at the feto-maternal interface. Am J Reprod Immunol $68,8-27$.

King, A., Allan, D.S., Bowen, M., Powis, S.J., Joseph, S., Verma, S., Hiby, S.E., McMichael, A.J., Loke, Y.W., and Braud, V.M. (2000a). HLA-E is expressed on trophoblast and interacts with CD94/NKG2 receptors on decidual NK cells. Eur J Immunol 30, 1623-1631.

King, A., Balendran, N., Wooding, P., Carter, N.P., and Loke, Y.W. (1991). CD3- leukocytes present in the human uterus during early placentation: phenotypic and morphologic characterization of the CD56++ population. Dev Immunol 1, 169-190.

King, A., Burrows, T.D., Hiby, S.E., Bowen, J.M., Joseph, S., Verma, S., Lim, P.B., Gardner, L., Le Bouteiller, P., Ziegler, A., Uchanska-Ziegler, B., and Loke, Y.W. (2000b). Surface expression of HLA-C antigen by human extravillous trophoblast. Placenta 21, 376-387.

King, A., Thomas, L., and Bischof, P. (2000c). Cell culture models of trophoblast II: trophoblast cell lines--a workshop report. Placenta 21 Suppl A, S113-119.

Kittan, N.A., Allen, R.M., Dhaliwal, A., Cavassani, K.A., Schaller, M., Gallagher, K.A., Carson, W.F.t., Mukherjee, S., Grembecka, J., Cierpicki, T., Jarai, G., Westwick, J., Kunkel, S.L., and Hogaboam, C.M. (2013). Cytokine induced phenotypic and epigenetic signatures are key to establishing specific macrophage phenotypes. PLoS One 8 , e78045. 
Kleinman, H.K., and Martin, G.R. (2005). Matrigel: basement membrane matrix with biological activity. Semin Cancer Biol 15, 378-386.

Konstantinov, S.R., Smidt, H., de Vos, W.M., Bruijns, S.C., Singh, S.K., Valence, F., Molle, D., Lortal, S., Altermann, E., Klaenhammer, T.R., and van Kooyk, Y. (2008). S layer protein A of Lactobacillus acidophilus NCFM regulates immature dendritic cell and T cell functions. Proc Natl Acad Sci U S A 105, 19474-19479.

Koopman, L.A., Kopcow, H.D., Rybalov, B., Boyson, J.E., Orange, J.S., Schatz, F., Masch, R., Lockwood, C.J., Schachter, A.D., Park, P.J., and Strominger, J.L. (2003). Human decidual natural killer cells are a unique NK cell subset with immunomodulatory potential. J Exp Med 198, 1201-1212.

Kopcow, H.D., Allan, D.S., Chen, X., Rybalov, B., Andzelm, M.M., Ge, B., and Strominger, J.L. (2005). Human decidual NK cells form immature activating synapses and are not cytotoxic. Proc Natl Acad Sci U S A 102, 15563-15568.

Krausgruber, T., Blazek, K., Smallie, T., Alzabin, S., Lockstone, H., Sahgal, N., Hussell, T., Feldmann, M., and Udalova, I.A. (2011). IRF5 promotes inflammatory macrophage polarization and TH1-TH17 responses. Nat Immunol 12, 231-238.

Krishnan, L., Guilbert, L.J., Wegmann, T.G., Belosevic, M., and Mosmann, T.R. (1996). T helper 1 response against Leishmania major in pregnant C57BL/6 mice increases implantation failure and fetal resorptions. Correlation with increased IFN-gamma and TNF and reduced IL-10 production by placental cells. J Immunol 156, 653-662.

Lacey, D.C., Achuthan, A., Fleetwood, A.J., Dinh, H., Roiniotis, J., Scholz, G.M., Chang, M.W., Beckman, S.K., Cook, A.D., and Hamilton, J.A. (2012). Defining GM-CSF- and macrophage-CSF-dependent macrophage responses by in vitro models. J Immunol 188 , $5752-5765$.

Larsen, E.C., Christiansen, O.B., Kolte, A.M., and Macklon, N. (2013). New insights into mechanisms behind miscarriage. BMC medicine 11, 154.

Lash, G.E., Naruse, K., Innes, B.A., Robson, S.C., Searle, R.F., and Bulmer, J.N. (2010). Secretion of angiogenic growth factors by villous cytotrophoblast and extravillous trophoblast in early human pregnancy. Placenta $31,545-548$.

Lash, G.E., Schiessl, B., Kirkley, M., Innes, B.A., Cooper, A., Searle, R.F., Robson, S.C., and Bulmer, J.N. (2006). Expression of angiogenic growth factors by uterine natural killer cells during early pregnancy. J Leukoc Biol 80, 572-580.

Laskarin, G., Cupurdija, K., Tokmadzic, V.S., Dorcic, D., Dupor, J., Juretic, K., Strbo, N., Crncic, T.B., Marchezi, F., Allavena, P., Mantovani, A., Randic, L., and Rukavina, D. (2005). The presence of functional mannose receptor on macrophages at the maternal-fetal interface. Hum Reprod 20, 1057-1066.

Li, C., Houser, B.L., Nicotra, M.L., and Strominger, J.L. (2009). HLA-G homodimer-induced cytokine secretion through HLA-G receptors on human decidual macrophages and natural killer cells. Proc Natl Acad Sci U S A 106, 5767-5772. 
Li, X., Mai, J., Virtue, A., Yin, Y., Gong, R., Sha, X., Gutchigian, S., Frisch, A., Hodge, I., Jiang, X., Wang, H., and Yang, X.F. (2012). IL-35 is a novel responsive anti-inflammatory cytokine--a new system of categorizing anti-inflammatory cytokines. PLoS One 7, e33628.

Lidstrom, C., Matthiesen, L., Berg, G., Sharma, S., Ernerudh, J., and Ekerfelt, C. (2003). Cytokine secretion patterns of NK cells and macrophages in early human pregnancy decidua and blood: implications for suppressor macrophages in decidua. Am J Reprod Immunol 50, 444-452.

Lin, H., Lee, E., Hestir, K., Leo, C., Huang, M., Bosch, E., Halenbeck, R., Wu, G., Zhou, A., Behrens, D., Hollenbaugh, D., Linnemann, T., Qin, M., Wong, J., Chu, K., Doberstein, S.K., and Williams, L.T. (2008). Discovery of a cytokine and its receptor by functional screening of the extracellular proteome. Science 320, 807-811.

Lin, H., Mosmann, T.R., Guilbert, L., Tuntipopipat, S., and Wegmann, T.G. (1993). Synthesis of T helper 2-type cytokines at the maternal-fetal interface. J Immunol 151, 45624573 .

Liu, W., Putnam, A.L., Xu-Yu, Z., Szot, G.L., Lee, M.R., Zhu, S., Gottlieb, P.A., Kapranov, P., Gingeras, T.R., Fazekas de St Groth, B., Clayberger, C., Soper, D.M., Ziegler, S.F., and Bluestone, J.A. (2006). CD127 expression inversely correlates with FoxP3 and suppressive function of human CD4+ T reg cells. J Exp Med 203, 1701-1711.

Liu, Y., Wu, Y., Wang, Y., Cai, Y., Hu, B., Bao, G., Fang, H., Zhao, L., Ma, S., Cheng, Q., Song, Y., Liu, Y., Zhu, Z., Chang, H., Yu, X., Sun, A., Zhang, Y., Vignali, D.A., Wu, D., and Liu, H. (2015). IL-35 mitigates murine acute graft-versus-host disease with retention of graftversus-leukemia effects. Leukemia : official journal of the Leukemia Society of America, Leukemia Research Fund, U.K 29, 939-946.

Llop-Guevara, A., Chu, D.K., Walker, T.D., Goncharova, S., Fattouh, R., Silver, J.S., Moore, C.L., Xie, J.L., O'Byrne, P.M., Coyle, A.J., Kolbeck, R., Humbles, A.A., Stampfli, M.R., and Jordana, M. (2014). A GM-CSF/IL-33 pathway facilitates allergic airway responses to subthreshold house dust mite exposure. PLoS One 9, e88714.

Lockwood, C.J., Huang, S.J., Chen, C.P., Huang, Y., Xu, J., Faramarzi, S., Kayisli, O., Kayisli, U., Koopman, L., Smedts, D., Buchwalder, L.F., and Schatz, F. (2013). Decidual cell regulation of natural killer cell-recruiting chemokines: implications for the pathogenesis and prediction of preeclampsia. Am J Pathol 183, 841-856.

Lockwood, C.J., Matta, P., Krikun, G., Koopman, L.A., Masch, R., Toti, P., Arcuri, F., Huang, S.T., Funai, E.F., and Schatz, F. (2006). Regulation of monocyte chemoattractant protein-1 expression by tumor necrosis factor-alpha and interleukin-1beta in first trimester human decidual cells: implications for preeclampsia. Am J Pathol 168, 445-452.

Luppi, P., Haluszczak, C., Betters, D., Richard, C.A.H., Trucco, M., and DeLoia, J.A. (2002). Monocytes are progressively activated in the circulation of pregnant women. Journal of Leukocyte Biology 72, 874-884.

Lyons, A.B. (2000). Analysing cell division in vivo and in vitro using flow cytometric measurement of CFSE dye dilution. J Immunol Methods 243, 147-154. 
Male, V., Hughes, T., McClory, S., Colucci, F., Caligiuri, M.A., and Moffett, A. (2010). Immature NK cells, capable of producing IL-22, are present in human uterine mucosa. J Immunol 185, 3913-3918.

Manaster, I., and Mandelboim, O. (2010). The unique properties of uterine NK cells. Am J Reprod Immunol 63, 434-444.

Manaster, I., Mizrahi, S., Goldman-Wohl, D., Sela, H.Y., Stern-Ginossar, N., Lankry, D., Gruda, R., Hurwitz, A., Bdolah, Y., Haimov-Kochman, R., Yagel, S., and Mandelboim, O. (2008). Endometrial NK cells are special immature cells that await pregnancy. J Immunol $181,1869-1876$.

Mantovani, A., Sica, A., Sozzani, S., Allavena, P., Vecchi, A., and Locati, M. (2004). The chemokine system in diverse forms of macrophage activation and polarization. Trends Immunol 25, 677-686.

Mao, H., Gao, W., Ma, C., Sun, J., Liu, J., Shao, Q., Song, B., and Qu, X. (2013). Human placental trophoblasts express the immunosuppressive cytokine IL-35. Hum Immunol 74, 872-877.

Martinez, F.O., and Gordon, S. (2014). The M1 and M2 paradigm of macrophage activation: time for reassessment. F1000prime reports 6, 13.

Martinez, F.O., Gordon, S., Locati, M., and Mantovani, A. (2006). Transcriptional profiling of the human monocyte-to-macrophage differentiation and polarization: new molecules and patterns of gene expression. J Immunol 177, 7303-7311.

Martinez, F.O., Helming, L., and Gordon, S. (2009). Alternative activation of macrophages: an immunologic functional perspective. Annu Rev Immunol 27, 451-483.

Masteller, E.L., and Wong, B.R. (2014). Targeting IL-34 in chronic inflammation. Drug discovery today $19,1212-1216$.

Matthiesen, L., Kalkunte, S., and Sharma, S. (2012). Multiple pregnancy failures: an immunological paradigm. Am J Reprod Immunol 67, 334-340.

McMaster, M.T., Librach, C.L., Zhou, Y., Lim, K.H., Janatpour, M.J., DeMars, R., Kovats, S., Damsky, C., and Fisher, S.J. (1995). Human placental HLA-G expression is restricted to differentiated cytotrophoblasts. J Immunol 154, 3771-3778.

Melgert, B.N., Spaans, F., Borghuis, T., Klok, P.A., Groen, B., Bolt, A., de Vos, P., van Pampus, M.G., Wong, T.Y., van Goor, H., Bakker, W.W., and Faas, M.M. (2012). Pregnancy and preeclampsia affect monocyte subsets in humans and rats. PLoS One 7, e45229.

Melief, S.M., Schrama, E., Brugman, M.H., Tiemessen, M.M., Hoogduijn, M.J., Fibbe, W.E., and Roelofs, H. (2013). Multipotent stromal cells induce human regulatory T cells through a novel pathway involving skewing of monocytes toward anti-inflammatory macrophages.

Stem cells 31, 1980-1991.

Menzies, F.M., Henriquez, F.L., Alexander, J., and Roberts, C.W. (2011). Selective inhibition and augmentation of alternative macrophage activation by progesterone. Immunology 134, 281-291. 
Miller, L., and Hunt, J.S. (1998). Regulation of TNF-alpha production in activated mouse macrophages by progesterone. J Immunol 160, 5098-5104.

Miller, R.K., Genbacev, O., Turner, M.A., Aplin, J.D., Caniggia, I., and Huppertz, B. (2005). Human placental explants in culture: approaches and assessments. Placenta 26, 439-448.

Mills, C.D., Kincaid, K., Alt, J.M., Heilman, M.J., and Hill, A.M. (2000). M-1/M-2 macrophages and the Th1/Th2 paradigm. J Immunol 164, 6166-6173.

Milne, S.A., Henderson, T.A., Kelly, R.W., Saunders, P.T., Baird, D.T., and Critchley, H.O. (2005). Leukocyte populations and steroid receptor expression in human first-trimester decidua; regulation by antiprogestin and prostaglandin E analog. J Clin Endocrinol Metab 90, 4315-4321.

Miyara, M., and Sakaguchi, S. (2011). Human FoxP3(+)CD4(+) regulatory T cells: their knowns and unknowns. Immunol Cell Biol 89, 346-351.

Miyaura, H., and Iwata, M. (2002). Direct and indirect inhibition of Th1 development by progesterone and glucocorticoids. J Immunol 168, 1087-1094.

Mizuno, M., Aoki, K., and Kimbara, T. (1994). Functions of macrophages in human decidual tissue in early pregnancy. Am J Reprod Immunol 31, 180-188.

Mjosberg, J., Berg, G., Ernerudh, J., and Ekerfelt, C. (2007). CD4+ CD25+ regulatory T cells in human pregnancy: development of a Treg-MLC-ELISPOT suppression assay and indications of paternal specific Tregs. Immunology 120, 456-466.

Mjosberg, J., Berg, G., Jenmalm, M.C., and Ernerudh, J. (2010). FOXP3+ regulatory T cells and $\mathrm{T}$ helper $1, \mathrm{~T}$ helper 2 , and $\mathrm{T}$ helper 17 cells in human early pregnancy decidua. Biol Reprod 82, 698-705.

Mjosberg, J., Svensson, J., Johansson, E., Hellstrom, L., Casas, R., Jenmalm, M.C., Boij, R., Matthiesen, L., Jonsson, J.I., Berg, G., and Ernerudh, J. (2009). Systemic reduction of functionally suppressive CD4dimCD25highFoxp3+ Tregs in human second trimester pregnancy is induced by progesterone and 17beta-estradiol. J Immunol 183, 759-769.

Moldenhauer, L.M., Keenihan, S.N., Hayball, J.D., and Robertson, S.A. (2010). GM-CSF is an essential regulator of $\mathrm{T}$ cell activation competence in uterine dendritic cells during early pregnancy in mice. J Immunol 185, 7085-7096.

Moller, H.J. (2012). Soluble CD163. Scandinavian journal of clinical and laboratory investigation $72,1-13$.

Mor, G., and Abrahams, V.M. (2003). Potential role of macrophages as immunoregulators of pregnancy. Reprod Biol Endocrinol 1, 119.

Morales-Prieto, D.M., Chaiwangyen, W., Ospina-Prieto, S., Schneider, U., Herrmann, J., Gruhn, B., and Markert, U.R. (2012). MicroRNA expression profiles of trophoblastic cells. Placenta 33, 725-734.

Morstyn, G., and Burgess, A.W. (1988). Hemopoietic growth factors: a review. Cancer Res $48,5624-5637$. 
Mosser, D.M., and Edwards, J.P. (2008). Exploring the full spectrum of macrophage activation. Nat Rev Immunol 8, 958-969.

Mosser, D.M., and Zhang, X. (2008). Interleukin-10: new perspectives on an old cytokine. Immunol Rev 226, 205-218.

Munn, D.H., Shafizadeh, E., Attwood, J.T., Bondarev, I., Pashine, A., and Mellor, A.L. (1999). Inhibition of T cell proliferation by macrophage tryptophan catabolism. J Exp Med 189, 1363-1372.

Munn, D.H., Zhou, M., Attwood, J.T., Bondarev, I., Conway, S.J., Marshall, B., Brown, C., and Mellor, A.L. (1998). Prevention of allogeneic fetal rejection by tryptophan catabolism. Science 281, 1191-1193.

Murray, P.J., Allen, J.E., Biswas, S.K., Fisher, E.A., Gilroy, D.W., Goerdt, S., Gordon, S., Hamilton, J.A., Ivashkiv, L.B., Lawrence, T., Locati, M., Mantovani, A., Martinez, F.O., Mege, J.L., Mosser, D.M., Natoli, G., Saeij, J.P., Schultze, J.L., Shirey, K.A., Sica, A., Suttles, J., Udalova, I., van Ginderachter, J.A., Vogel, S.N., and Wynn, T.A. (2014). Macrophage activation and polarization: nomenclature and experimental guidelines. Immunity 41, 14-20.

Murray, P.J., and Wynn, T.A. (2011). Protective and pathogenic functions of macrophage subsets. Nat Rev Immunol 11, 723-737.

Nagamatsu, T., and Schust, D.J. (2010a). The contribution of macrophages to normal and pathological pregnancies. Am J Reprod Immunol 63, 460-471.

Nagamatsu, T., and Schust, D.J. (2010b). The immunomodulatory roles of macrophages at the maternal-fetal interface. Reprod Sci 17, 209-218.

Nagamatsu, T., Schust, D.J., Sugimoto, J., and Barrier, B.F. (2009). Human decidual stromal cells suppress cytokine secretion by allogenic CD4+ T cells via PD-1 ligand interactions. Hum Reprod 24, 3160-3171.

Nagler, A., Lanier, L.L., Cwirla, S., and Phillips, J.H. (1989). Comparative studies of human FcRIII-positive and negative natural killer cells. J Immunol 143, 3183-3191.

Nancy, P., and Erlebacher, A. (2014). T cell behavior at the maternal-fetal interface. Int J Dev Biol 58, 189-198.

Nancy, P., Tagliani, E., Tay, C.S., Asp, P., Levy, D.E., and Erlebacher, A. (2012). Chemokine gene silencing in decidual stromal cells limits $\mathrm{T}$ cell access to the maternal-fetal interface. Science 336, 1317-1321.

Nguyen, K.D., Vanichsarn, C., Fohner, A., and Nadeau, K.C. (2009). Selective deregulation in chemokine signaling pathways of CD4+CD25(hi)CD127(lo)/(-) regulatory T cells in human allergic asthma. J Allergy Clin Immunol 123, 933-939 e910.

Niedbala, W., Wei, X.Q., Cai, B., Hueber, A.J., Leung, B.P., McInnes, I.B., and Liew, F.Y. (2007). IL-35 is a novel cytokine with therapeutic effects against collagen-induced arthritis through the expansion of regulatory T cells and suppression of Th17 cells. Eur J Immunol 37, 3021-3029. 
Noster, R., Riedel, R., Mashreghi, M.F., Radbruch, H., Harms, L., Haftmann, C., Chang, H.D., Radbruch, A., and Zielinski, C.E. (2014). IL-17 and GM-CSF expression are antagonistically regulated by human $\mathrm{T}$ helper cells. Science translational medicine 6 , $241 \mathrm{ra} 280$

Olson, B.M., Jankowska-Gan, E., Becker, J.T., Vignali, D.A., Burlingham, W.J., and McNeel, D.G. (2012). Human prostate tumor antigen-specific CD8+ regulatory T cells are inhibited by CTLA-4 or IL-35 blockade. J Immunol 189, 5590-5601.

Ostensen, M., Aune, B., and Husby, G. (1983). Effect of pregnancy and hormonal changes on the activity of rheumatoid arthritis. Scand.J Rheumatol. 12, 69-72.

Pampfer, S., Daiter, E., Barad, D., and Pollard, J.W. (1992). Expression of the colonystimulating factor-1 receptor (c-fms proto-oncogene product) in the human uterus and placenta. Biol Reprod 46, 48-57.

Park, H., Li, Z., Yang, X.O., Chang, S.H., Nurieva, R., Wang, Y.H., Wang, Y., Hood, L., Zhu, Z., Tian, Q., and Dong, C. (2005). A distinct lineage of CD4 T cells regulates tissue inflammation by producing interleukin 17. Nat Immunol 6, 1133-1141.

Piao, L., Chen, C.P., Yeh, C.C., Basar, M., Masch, R., Cheng, Y.C., Lockwood, C.J., Schatz, F., and Huang, S.J. (2015). Chinese herbal medicine for miscarriage affects decidual microenvironment and fetal growth. Placenta 36, 559-566.

Piccinni, M.P., Scaletti, C., Vultaggio, A., Maggi, E., and Romagnani, S. (2001). Defective production of LIF, M-CSF and Th2-type cytokines by T cells at fetomaternal interface is associated with pregnancy loss. J Reprod Immunol 52, 35-43.

Pitman, H., Innes, B.A., Robson, S.C., Bulmer, J.N., and Lash, G.E. (2013). Altered expression of interleukin-6, interleukin-8 and their receptors in decidua of women with sporadic miscarriage. Hum Reprod 28, 2075-2086.

Polanczyk, M.J., Hopke, C., Vandenbark, A.A., and Offner, H. (2006). Estrogen-mediated immunomodulation involves reduced activation of effector T cells, potentiation of Treg cells, and enhanced expression of the PD-1 costimulatory pathway. Journal of neuroscience research 84, 370-378.

Pollard, J.W. (2004). Tumour-educated macrophages promote tumour progression and metastasis. Nature reviews. Cancer 4, 71-78.

Pollard, J.W. (2009). Trophic macrophages in development and disease. Nat Rev Immunol 9, 259-270.

Pollard, J.W., Hunt, J.S., Wiktor-Jedrzejczak, W., and Stanley, E.R. (1991). A pregnancy defect in the osteopetrotic (op/op) mouse demonstrates the requirement for CSF-1 in female fertility. Dev Biol 148, 273-283.

Prins, J.R., Faas, M.M., Melgert, B.N., Huitema, S., Timmer, A., Hylkema, M.N., and Erwich, J.J. (2012). Altered expression of immune-associated genes in first-trimester human decidua of pregnancies later complicated with hypertension or foetal growth restriction. Placenta 33, 453-455. 
Puig-Kroger, A., Serrano-Gomez, D., Caparros, E., Dominguez-Soto, A., Relloso, M., Colmenares, M., Martinez-Munoz, L., Longo, N., Sanchez-Sanchez, N., Rincon, M., Rivas, L., Sanchez-Mateos, P., Fernandez-Ruiz, E., and Corbi, A.L. (2004). Regulated expression of the pathogen receptor dendritic cell-specific intercellular adhesion molecule 3 (ICAM-3)grabbing nonintegrin in THP-1 human leukemic cells, monocytes, and macrophages. J Biol Chem 279, 25680-25688.

Qin, S., Rottman, J.B., Myers, P., Kassam, N., Weinblatt, M., Loetscher, M., Koch, A.E., Moser, B., and Mackay, C.R. (1998). The chemokine receptors CXCR3 and CCR5 mark subsets of T cells associated with certain inflammatory reactions. J Clin Invest 101, 746-754.

Qiu, X., Zhu, L., and Pollard, J.W. (2009). Colony-stimulating factor-1-dependent macrophage functions regulate the maternal decidua immune responses against Listeria monocytogenes infections during early gestation in mice. Infect Immun 77, 85-97.

Quatromoni, J.G., Singhal, S., Bhojnagarwala, P., Hancock, W.W., Albelda, S.M., and Eruslanov, E. (2015). An optimized disaggregation method for human lung tumors that preserves the phenotype and function of the immune cells. J Leukoc Biol 97, 201-209.

Raghupathy, R. (1997). Th1-type immunity is incompatible with successful pregnancy. Immunol Today $18,478-482$.

Rao, P.E., Petrone, A.L., and Ponath, P.D. (2005). Differentiation and expansion of T cells with regulatory function from human peripheral lymphocytes by stimulation in the presence of TGF-\{beta\}. J Immunol 174, 1446-1455.

Redman, C.W., and Sargent, I.L. (2010). Immunology of pre-eclampsia. Am J Reprod Immunol 63, 534-543.

Renaud, S.J., Macdonald-Goodfellow, S.K., and Graham, C.H. (2007). Coordinated regulation of human trophoblast invasiveness by macrophages and interleukin 10. Biol Reprod 76, 448-454.

Renaud, S.J., Postovit, L.M., Macdonald-Goodfellow, S.K., McDonald, G.T., Caldwell, J.D., and Graham, C.H. (2005). Activated macrophages inhibit human cytotrophoblast invasiveness in vitro. Biol Reprod 73, 237-243.

Rieger, L., Hofmeister, V., Probe, C., Dietl, J., Weiss, E.H., Steck, T., and Kammerer, U. (2002). Th1- and Th2-like cytokine production by first trimester decidual large granular lymphocytes is influenced by HLA-G and HLA-E. Mol Hum Reprod 8, 255-261.

Robertson, S.A., Care, A.S., and Skinner, R.J. (2007). Interleukin 10 regulates inflammatory cytokine synthesis to protect against lipopolysaccharide-induced abortion and fetal growth restriction in mice. Biol Reprod 76, 738-748.

Robertson, S.A., Prins, J.R., Sharkey, D.J., and Moldenhauer, L.M. (2013). Seminal fluid and the generation of regulatory T cells for embryo implantation. Am J Reprod Immunol 69, 315330 .

Roca, H., Varsos, Z.S., Sud, S., Craig, M.J., Ying, C., and Pienta, K.J. (2009). CCL2 and interleukin-6 promote survival of human $\mathrm{CD} 11 \mathrm{~b}+$ peripheral blood mononuclear cells and induce M2-type macrophage polarization. J Biol Chem 284, 34342-34354. 
Romero, R., Dey, S.K., and Fisher, S.J. (2014). Preterm labor: one syndrome, many causes. Science $345,760-765$.

Romero, R., Espinoza, J., Goncalves, L.F., Kusanovic, J.P., Friel, L.A., and Nien, J.K. (2006). Inflammation in preterm and term labour and delivery. Seminars in fetal \& neonatal medicine 11,317-326.

Rowe, J.H., Ertelt, J.M., Xin, L., and Way, S.S. (2012a). Listeria monocytogenes cytoplasmic entry induces fetal wastage by disrupting maternal Foxp3+ regulatory T cell-sustained fetal tolerance. PLoS Pathog 8, e1002873.

Rowe, J.H., Ertelt, J.M., Xin, L., and Way, S.S. (2012b). Pregnancy imprints regulatory memory that sustains anergy to fetal antigen. Nature 490, 102-106.

Sacks, G., Sargent, I., and Redman, C. (1999). An innate view of human pregnancy. Immunol Today 20, 114-118.

Sacks, G.P., Clover, L.M., Bainbridge, D.R., Redman, C.W., and Sargent, I.L. (2001). Th1 and Th2 cytokine production by villous and extravillous cytotrophoblast. Placenta 22, 550559.

Sacks, G.P., Redman, C.W.G., and Sargent, I.L. (2003). Monocytes are primed to produce the Th1 type cytokine IL-12 in normal human pregnancy: an intracellular flow cytometric analysis of peripheral blood mononuclear cells. Clinical and Experimental Immunology 131, 490-497.

Sacks, G.P., Studena, K., Sargent, I.L., and Redman, C.W.G. (1998). Normal pregnancy and preeclampsia both produce inflammatory changes in peripheral blood leukocytes akin to those of sepsis. American Journal of Obstetrics and Gynecology 179, 80-86.

Saito, S., Nakashima, A., Shima, T., and Ito, M. (2010). Th1/Th2/Th17 and regulatory T-cell paradigm in pregnancy. Am J Reprod Immunol 63, 601-610.

Saito, S., Sakai, M., Sasaki, Y., Tanebe, K., Tsuda, H., and Michimata, T. (1999a). Quantitative analysis of peripheral blood Th0, Th1, Th2 and the Th1:Th2 cell ratio during normal pregnancy and preeclampsia. Clinical and Experimental Immunology 117, 550-555.

Saito, S., Tsukaguchi, N., Hasegawa, T., Michimata, T., Tsuda, H., and Narita, N. (1999b). Distribution of Th1, Th2, and Th0 and the Th1/Th2 cell ratios in human peripheral and endometrial T cells. American Journal of Reproductive Immunology 42, 240-245.

Sakaguchi, S., Sakaguchi, N., Asano, M., Itoh, M., and Toda, M. (1995). Immunologic selftolerance maintained by activated T cells expressing IL-2 receptor alpha-chains (CD25). Breakdown of a single mechanism of self-tolerance causes various autoimmune diseases. J Immunol 155, 1151-1164.

Samstein, R.M., Josefowicz, S.Z., Arvey, A., Treuting, P.M., and Rudensky, A.Y. (2012). Extrathymic generation of regulatory $\mathrm{T}$ cells in placental mammals mitigates maternal-fetal conflict. Cell 150, 29-38.

Sanchez-Rodriguez, E.N., Nava-Salazar, S., Mendoza-Rodriguez, C.A., Moran, C., RomeroArauz, J.F., Ortega, E., Granados, J., Cervantes-Peredo, A., and Cerbon, M. (2011). 
Persistence of decidual NK cells and KIR genotypes in healthy pregnant and preeclamptic women: a case-control study in the third trimester of gestation. Reprod Biol Endocrinol 9, 8.

Santner-Nanan, B., Straubinger, K., Hsu, P., Parnell, G., Tang, B., Xu, B., Makris, A., Hennessy, A., Peek, M.J., Busch, D.H., da Costa, C.P., and Nanan, R. (2013). Fetal-maternal alignment of regulatory T cells correlates with IL-10 and Bcl-2 upregulation in pregnancy. $\mathrm{J}$ Immunol 191, 145-153.

Sarris, M., Andersen, K.G., Randow, F., Mayr, L., and Betz, A.G. (2008). Neuropilin-1 expression on regulatory $\mathrm{T}$ cells enhances their interactions with dendritic cells during antigen recognition. Immunity 28, 402-413.

Sasaki, Y., Sakai, M., Miyazaki, S., Higuma, S., Shiozaki, A., and Saito, S. (2004). Decidual and peripheral blood $\mathrm{CD} 4+\mathrm{CD} 25+$ regulatory $\mathrm{T}$ cells in early pregnancy subjects and spontaneous abortion cases. Mol Hum Reprod 10, 347-353.

Savage, N.D., de Boer, T., Walburg, K.V., Joosten, S.A., van Meijgaarden, K., Geluk, A., and Ottenhoff, T.H. (2008). Human anti-inflammatory macrophages induce Foxp3+ GITR+ $\mathrm{CD} 25+$ regulatory T cells, which suppress via membrane-bound TGFbeta-1. J Immunol 181, 2220-2226.

Sayama, S., Nagamatsu, T., Schust, D.J., Itaoka, N., Ichikawa, M., Kawana, K., Yamashita, T., Kozuma, S., and Fujii, T. (2013). Human decidual macrophages suppress IFN-gamma production by $\mathrm{T}$ cells through costimulatory $\mathrm{B} 7-\mathrm{H} 1: \mathrm{PD}-1$ signaling in early pregnancy. $\mathrm{J}$ Reprod Immunol 100, 109-117.

Schonkeren, D., van der Hoorn, M.L., Khedoe, P., Swings, G., van Beelen, E., Claas, F., van Kooten, C., de Heer, E., and Scherjon, S. (2011). Differential distribution and phenotype of decidual macrophages in preeclamptic versus control pregnancies. Am J Pathol 178, 709-717.

Schraufstatter, I.U., Zhao, M., Khaldoyanidi, S.K., and Discipio, R.G. (2012). The chemokine CCL18 causes maturation of cultured monocytes to macrophages in the M2 spectrum. Immunology 135, 287-298.

Schulz, C., Gomez Perdiguero, E., Chorro, L., Szabo-Rogers, H., Cagnard, N., Kierdorf, K., Prinz, M., Wu, B., Jacobsen, S.E., Pollard, J.W., Frampton, J., Liu, K.J., and Geissmann, F. (2012). A lineage of myeloid cells independent of Myb and hematopoietic stem cells. Science $336,86-90$.

Schumacher, A., Brachwitz, N., Sohr, S., Engeland, K., Langwisch, S., Dolaptchieva, M., Alexander, T., Taran, A., Malfertheiner, S.F., Costa, S.D., Zimmermann, G., Nitschke, C., Volk, H.D., Alexander, H., Gunzer, M., and Zenclussen, A.C. (2009). Human chorionic gonadotropin attracts regulatory $\mathrm{T}$ cells into the fetal-maternal interface during early human pregnancy. J Immunol 182, 5488-5497.

Schumacher, A., Heinze, K., Witte, J., Poloski, E., Linzke, N., Woidacki, K., and Zenclussen, A.C. (2013). Human chorionic gonadotropin as a central regulator of pregnancy immune tolerance. J Immunol 190, 2650-2658.

Schumacher, A., Poloski, E., Sporke, D., and Zenclussen, A.C. (2014). Luteinizing hormone contributes to fetal tolerance by regulating adaptive immune responses. Am J Reprod Immunol 71, 434-440. 
Segaliny, A.I., Mohamadi, A., Dizier, B., Lokajczyk, A., Brion, R., Lanel, R., Amiaud, J., Charrier, C., Boisson-Vidal, C., and Heymann, D. (2014). Interleukin-34 promotes tumor progression and metastatic process in osteosarcoma through induction of angiogenesis and macrophage recruitment. Int J Cancer. DOI: 10.1002/ijc.29376.

Selmani, Z., Naji, A., Zidi, I., Favier, B., Gaiffe, E., Obert, L., Borg, C., Saas, P., Tiberghien, P., Rouas-Freiss, N., Carosella, E.D., and Deschaseaux, F. (2008). Human leukocyte antigenG5 secretion by human mesenchymal stem cells is required to suppress $\mathrm{T}$ lymphocyte and natural killer function and to induce CD4+CD25highFOXP3+ regulatory T cells. Stem cells $26,212-222$.

Shen, P., Roch, T., Lampropoulou, V., O'Connor, R.A., Stervbo, U., Hilgenberg, E., Ries, S., Dang, V.D., Jaimes, Y., Daridon, C., Li, R., Jouneau, L., Boudinot, P., Wilantri, S., Sakwa, I., Miyazaki, Y., Leech, M.D., McPherson, R.C., Wirtz, S., Neurath, M., Hoehlig, K., Meinl, E., Grutzkau, A., Grun, J.R., Horn, K., Kuhl, A.A., Dorner, T., Bar-Or, A., Kaufmann, S.H., Anderton, S.M., and Fillatreau, S. (2014). IL-35-producing B cells are critical regulators of immunity during autoimmune and infectious diseases. Nature 507, 366-370.

Shi, C., and Pamer, E.G. (2011). Monocyte recruitment during infection and inflammation. Nat Rev Immunol 11, 762-774.

Sibai, B., Dekker, G., and Kupferminc, M. (2005). Pre-eclampsia. Lancet 365, 785-799.

Sica, A., and Mantovani, A. (2012). Macrophage plasticity and polarization: in vivo veritas. J Clin Invest 122, 787-795.

Sierra-Filardi, E., Nieto, C., Dominguez-Soto, A., Barroso, R., Sanchez-Mateos, P., PuigKroger, A., Lopez-Bravo, M., Joven, J., Ardavin, C., Rodriguez-Fernandez, J.L., SanchezTorres, C., Mellado, M., and Corbi, A.L. (2014). CCL2 shapes macrophage polarization by GM-CSF and M-CSF: identification of CCL2/CCR2-dependent gene expression profile. J Immunol 192, 3858-3867.

Simpson, H., Robson, S.C., Bulmer, J.N., Barber, A., and Lyall, F. (2002). Transforming growth factor beta expression in human placenta and placental bed during early pregnancy. Placenta 23, 44-58.

Singh, U., Nicholson, G., Urban, B.C., Sargent, I.L., Kishore, U., and Bernal, A.L. (2005). Immunological properties of human decidual macrophages--a possible role in intrauterine immunity. Reproduction 129, 631-637.

Smith, S.D., Dunk, C.E., Aplin, J.D., Harris, L.K., and Jones, R.L. (2009). Evidence for immune cell involvement in decidual spiral arteriole remodeling in early human pregnancy. Am J Pathol 174, 1959-1971.

Smits, H.H., Engering, A., van der Kleij, D., de Jong, E.C., Schipper, K., van Capel, T.M., Zaat, B.A., Yazdanbakhsh, M., Wierenga, E.A., van Kooyk, Y., and Kapsenberg, M.L. (2005). Selective probiotic bacteria induce IL-10-producing regulatory T cells in vitro by modulating dendritic cell function through dendritic cell-specific intercellular adhesion molecule 3-grabbing nonintegrin. J Allergy Clin Immunol 115, 1260-1267.

Soilleux, E.J., Morris, L.S., Leslie, G., Chehimi, J., Luo, Q., Levroney, E., Trowsdale, J., Montaner, L.J., Doms, R.W., Weissman, D., Coleman, N., and Lee, B. (2002). Constitutive 
and induced expression of DC-SIGN on dendritic cell and macrophage subpopulations in situ and in vitro. J Leukoc Biol 71, 445-457.

Somerset, D.A., Zheng, Y., Kilby, M.D., Sansom, D.M., and Drayson, M.T. (2004). Normal human pregnancy is associated with an elevation in the immune suppressive CD25+ CD4+ regulatory T-cell subset. Immunology 112, 38-43.

Stanley, E., Lieschke, G.J., Grail, D., Metcalf, D., Hodgson, G., Gall, J.A., Maher, D.W., Cebon, J., Sinickas, V., and Dunn, A.R. (1994). Granulocyte/macrophage colony-stimulating factor-deficient mice show no major perturbation of hematopoiesis but develop a characteristic pulmonary pathology. Proc Natl Acad Sci U S A 91, 5592-5596.

Starkey, P.M., Sargent, I.L., and Redman, C.W. (1988). Cell populations in human early pregnancy decidua: characterization and isolation of large granular lymphocytes by flow cytometry. Immunology 65, 129-134.

Stein, M., Keshav, S., Harris, N., and Gordon, S. (1992). Interleukin 4 potently enhances murine macrophage mannose receptor activity: a marker of alternative immunologic macrophage activation. J Exp Med 176, 287-292.

Stenqvist, A.C., Chen, T., Hedlund, M., Dimova, T., Nagaeva, O., Kjellberg, L., Innala, E., and Mincheva-Nilsson, L. (2008). An efficient optimized method for isolation of villous trophoblast cells from human early pregnancy placenta suitable for functional and molecular studies. Am J Reprod Immunol 60, 33-42.

Stilund, M., Reuschlein, A.K., Christensen, T., Moller, H.J., Rasmussen, P.V., and Petersen, T. (2014). Soluble CD163 as a marker of macrophage activity in newly diagnosed patients with multiple sclerosis. PLoS One 9, e98588.

Stout, R.D., and Suttles, J. (2004). Functional plasticity of macrophages: reversible adaptation to changing microenvironments. J Leukoc Biol 76, 509-513.

Sulahian, T.H., Hogger, P., Wahner, A.E., Wardwell, K., Goulding, N.J., Sorg, C., Droste, A., Stehling, M., Wallace, P.K., Morganelli, P.M., and Guyre, P.M. (2000). Human monocytes express CD163, which is upregulated by IL-10 and identical to p155. Cytokine $12,1312-1321$.

Sulpice, E., Plouet, J., Berge, M., Allanic, D., Tobelem, G., and Merkulova-Rainon, T. (2008). Neuropilin-1 and neuropilin-2 act as coreceptors, potentiating proangiogenic activity. Blood 111, 2036-2045.

Svensson-Arvelund, J., and Ernerudh, J. (2015). The Role of Macrophages in Promoting and Maintaining Homeostasis at the Fetal-Maternal Interface. Am J Reprod Immunol. doi:10.1111/aji.12357

Svensson-Arvelund, J., Ernerudh, J., Buse, E., Cline, J.M., Haeger, J.D., Dixon, D., Markert, U.R., Pfarrer, C., Vos, P.D., and Faas, M.M. (2014). The Placenta in Toxicology. Part II:

Systemic and Local Immune Adaptations in Pregnancy. Toxicologic pathology 42, 327-338.

Svensson-Arvelund, J., Mehta, R.B., Lindau, R., Mirrasekhian, E., Rodriguez-Martinez, H., Berg, G., Lash, G.E., Jenmalm, M.C., and Ernerudh, J. (2015). The Human Fetal Placenta 
Promotes Tolerance against the Semiallogeneic Fetus by Inducing Regulatory T Cells and Homeostatic M2 Macrophages. J Immunol 194, 1534-1544.

Szabo, S.J., Kim, S.T., Costa, G.L., Zhang, X., Fathman, C.G., and Glimcher, L.H. (2000). A novel transcription factor, T-bet, directs Th1 lineage commitment. Cell 100, 655-669.

Taams, L.S., van Amelsfort, J.M., Tiemessen, M.M., Jacobs, K.M., de Jong, E.C., Akbar, A.N., Bijlsma, J.W., and Lafeber, F.P. (2005). Modulation of monocyte/macrophage function by human CD4+CD25+ regulatory T cells. Hum Immunol 66, 222-230.

Tacke, F., and Randolph, G.J. (2006). Migratory fate and differentiation of blood monocyte subsets. Immunobiology 211, 609-618.

Tagliani, E., Shi, C., Nancy, P., Tay, C.S., Pamer, E.G., and Erlebacher, A. (2011).

Coordinate regulation of tissue macrophage and dendritic cell population dynamics by CSF1. J Exp Med 208, 1901-1916.

Takahashi, H.K., Iwagaki, H., Mori, S., Yoshino, T., Tanaka, N., and Nishibori, M. (2005). Prostaglandins E1 and E2 inhibit lipopolysaccharide-induced interleukin-18 production in monocytes. European journal of pharmacology 517, 252-256.

Takahashi, H.K., Iwagaki, H., Tamura, R., Xue, D., Sano, M., Mori, S., Yoshino, T., Tanaka, N., and Nishibori, M. (2003). Unique regulation profile of prostaglandin e1 on adhesion molecule expression and cytokine production in human peripheral blood mononuclear cells. The Journal of pharmacology and experimental therapeutics 307, 1188-1195.

Tamimi, R.M., Brugge, J.S., Freedman, M.L., Miron, A., Iglehart, J.D., Colditz, G.A., and Hankinson, S.E. (2008). Circulating colony stimulating factor-1 and breast cancer risk. Cancer Res 68, 18-21.

Tan, C., Wei, L., Vistica, B.P., Shi, G., Wawrousek, E.F., and Gery, I. (2014). Phenotypes of Th lineages generated by the commonly used activation with anti-CD3/CD28 antibodies differ from those generated by the physiological activation with the specific antigen. Cellular \& molecular immunology 11, 305-313.

Tang, O.S., Gemzell-Danielsson, K., and Ho, P.C. (2007). Misoprostol: pharmacokinetic profiles, effects on the uterus and side-effects. International journal of gynaecology and obstetrics: the official organ of the International Federation of Gynaecology and Obstetrics 99 Suppl 2, S160-167.

Tang, Z., Niven-Fairchild, T., Tadesse, S., Norwitz, E.R., Buhimschi, C.S., Buhimschi, I.A., and Guller, S. (2013). Glucocorticoids enhance CD163 expression in placental Hofbauer cells. Endocrinology 154, 471-482.

Tarrade, A., Schoonjans, K., Pavan, L., Auwerx, J., Rochette-Egly, C., Evain-Brion, D., and Fournier, T. (2001). PPARgamma/RXRalpha heterodimers control human trophoblast invasion. J Clin Endocrinol Metab 86, 5017-5024.

Terayama, H., Yoshimoto, T., Hirai, S., Naito, M., Qu, N., Hatayama, N., Hayashi, S., Mitobe, K., Furusawa, J., Mizoguchi, I., Kezuka, T., Goto, H., Suyama, K., Moriyama, H., Sakabe, K., and Itoh, M. (2014). Contribution of IL-12/IL-35 common subunit p35 to maintaining the testicular immune privilege. PLoS One 9, e96120. 
Thaxton, J.E., Romero, R., and Sharma, S. (2009). TLR9 activation coupled to IL-10 deficiency induces adverse pregnancy outcomes. J Immunol 183, 1144-1154.

Tian, L., Humblet-Baron, S., and Liston, A. (2012). Immune tolerance: are regulatory T cell subsets needed to explain suppression of autoimmunity? BioEssays : news and reviews in molecular, cellular and developmental biology 34, 569-575.

Tiemessen, M.M., Jagger, A.L., Evans, H.G., van Herwijnen, M.J., John, S., and Taams, L.S. (2007). CD4+CD25+Foxp3+ regulatory T cells induce alternative activation of human monocytes/macrophages. Proc Natl Acad Sci U S A 104, 19446-19451.

Tilburgs, T., Roelen, D.L., van der Mast, B.J., de Groot-Swings, G.M., Kleijburg, C., Scherjon, S.A., and Claas, F.H. (2008). Evidence for a selective migration of fetus-specific $\mathrm{CD} 4+\mathrm{CD} 25$ bright regulatory $\mathrm{T}$ cells from the peripheral blood to the decidua in human pregnancy. J Immunol 180, 5737-5745.

Tilburgs, T., Roelen, D.L., van der Mast, B.J., van Schip, J.J., Kleijburg, C., de GrootSwings, G.M., Kanhai, H.H., Claas, F.H., and Scherjon, S.A. (2006). Differential distribution of CD4(+)CD25(bright) and CD8(+)CD28(-) T-cells in decidua and maternal blood during human pregnancy. Placenta 27 Suppl A, S47-53.

Tilburgs, T., and Strominger, J.L. (2013). CD8+ effector T cells at the fetal-maternal interface, balancing fetal tolerance and antiviral immunity. Am J Reprod Immunol 69, 395407.

Trundley, A., and Moffett, A. (2004). Human uterine leukocytes and pregnancy. Tissue Antigens 63, 1-12.

Vacca, P., Cantoni, C., Vitale, M., Prato, C., Canegallo, F., Fenoglio, D., Ragni, N., Moretta, L., and Mingari, M.C. (2010). Crosstalk between decidual NK and CD14+ myelomonocytic cells results in induction of Tregs and immunosuppression. Proc Natl Acad Sci U S A 107, 11918-11923.

Vacca, P., Moretta, L., Moretta, A., and Mingari, M.C. (2011a). Origin, phenotype and function of human natural killer cells in pregnancy. Trends Immunol 32, 517-523.

Vacca, P., Vitale, C., Montaldo, E., Conte, R., Cantoni, C., Fulcheri, E., Darretta, V., Moretta, L., and Mingari, M.C. (2011b). CD34+ hematopoietic precursors are present in human decidua and differentiate into natural killer cells upon interaction with stromal cells. Proc Natl Acad Sci U S A 108, 2402-2407.

Waiser, J., Bohler, T., Stoll, J., Schumann, B., Budde, K., and Neumayer, H.H. (2003). The immunosuppressive potential of misoprostol--efficacy and variability. Clin Immunol 109, 288-294.

Van den Heuvel, M.M., Tensen, C.P., van As, J.H., Van den Berg, T.K., Fluitsma, D.M., Dijkstra, C.D., Dopp, E.A., Droste, A., Van Gaalen, F.A., Sorg, C., Hogger, P., and Beelen, R.H. (1999). Regulation of CD 163 on human macrophages: cross-linking of CD163 induces signaling and activation. J Leukoc Biol 66, 858-866.

van Kooyk, Y., and Geijtenbeek, T.B. (2003). DC-SIGN: escape mechanism for pathogens. Nat Rev Immunol 3, 697-709. 
Wang, J., Ioan-Facsinay, A., van der Voort, E.I., Huizinga, T.W., and Toes, R.E. (2007). Transient expression of FOXP3 in human activated nonregulatory CD4+ T cells. Eur J Immunol 37, 129-138.

Wang, Y., Szretter, K.J., Vermi, W., Gilfillan, S., Rossini, C., Cella, M., Barrow, A.D., Diamond, M.S., and Colonna, M. (2012). IL-34 is a tissue-restricted ligand of CSF1R required for the development of Langerhans cells and microglia. Nat Immunol 13, 753-760.

Weber, C., Belge, K.U., von Hundelshausen, P., Draude, G., Steppich, B., Mack, M., Frankenberger, M., Weber, K.S., and Ziegler-Heitbrock, H.W. (2000). Differential chemokine receptor expression and function in human monocyte subpopulations. J Leukoc Biol 67, 699-704.

Veenstra van Nieuwenhoven, A.L., Bouman, A., Moes, H., Heineman, M.J., de Leij, L.F., Santema, J., and Faas, M.M. (2002). Cytokine production in natural killer cells and lymphocytes in pregnant women compared with women in the follicular phase of the ovarian cycle. Fertil.Steril. 77, 1032-1037.

Wegmann, T.G., Lin, H., Guilbert, L., and Mosmann, T.R. (1993). Bidirectional cytokine interactions in the maternal-fetal relationship: is successful pregnancy a TH2 phenomenon? Immunol Today 14, 353-356.

Verreck, F.A., de Boer, T., Langenberg, D.M., Hoeve, M.A., Kramer, M., Vaisberg, E., Kastelein, R., Kolk, A., de Waal-Malefyt, R., and Ottenhoff, T.H. (2004). Human IL-23producing type 1 macrophages promote but IL-10-producing type 2 macrophages subvert immunity to (myco)bacteria. Proc Natl Acad Sci U S A 101, 4560-4565.

Verreck, F.A., de Boer, T., Langenberg, D.M., van der Zanden, L., and Ottenhoff, T.H. (2006). Phenotypic and functional profiling of human proinflammatory type-1 and antiinflammatory type-2 macrophages in response to microbial antigens and IFN-gamma- and CD40L-mediated costimulation. J Leukoc Biol 79, 285-293.

Whitacre, C.C., Reingold, S.C., and O'Looney, P.A. (1999). A gender gap in autoimmunity. Science 283, 1277-1278.

Vignali, D.A., and Kuchroo, V.K. (2012). IL-12 family cytokines: immunological playmakers. Nat Immunol 13, 722-728.

Wiktor-Jedrzejczak, W., Bartocci, A., Ferrante, A.W., Jr., Ahmed-Ansari, A., Sell, K.W., Pollard, J.W., and Stanley, E.R. (1990). Total absence of colony-stimulating factor 1 in the macrophage-deficient osteopetrotic (op/op) mouse. Proc Natl Acad Sci U S A 87, 4828-4832.

Wiktor-Jedrzejczak, W., and Gordon, S. (1996). Cytokine regulation of the macrophage (M phi) system studied using the colony stimulating factor-1-deficient op/op mouse. Physiol Rev 76, 927-947.

Williams, P.J., Searle, R.F., Robson, S.C., Innes, B.A., and Bulmer, J.N. (2009). Decidual leucocyte populations in early to late gestation normal human pregnancy. J Reprod Immunol 82, 24-31. 
Wing, K., Onishi, Y., Prieto-Martin, P., Yamaguchi, T., Miyara, M., Fehervari, Z., Nomura, T., and Sakaguchi, S. (2008). CTLA-4 control over Foxp3+ regulatory T cell function.

Science 322, 271-275.

Vogel, I., Grove, J., Thorsen, P., Moestrup, S.K., Uldbjerg, N., and Moller, H.J. (2005). Preterm delivery predicted by soluble CD163 and CRP in women with symptoms of preterm delivery. Bjog 112, 737-742.

Workman, C.J., Szymczak-Workman, A.L., Collison, L.W., Pillai, M.R., and Vignali, D.A. (2009). The development and function of regulatory T cells. Cellular and molecular life sciences : CMLS 66, 2603-2622.

Wu, X., Jin, L.P., Yuan, M.M., Zhu, Y., Wang, M.Y., and Li, D.J. (2005). Human firsttrimester trophoblast cells recruit CD56brightCD16- NK cells into decidua by way of expressing and secreting of CXCL12/stromal cell-derived factor 1. J Immunol 175, 61-68.

Wynn, T.A., Chawla, A., and Pollard, J.W. (2013). Macrophage biology in development, homeostasis and disease. Nature 496, 445-455.

Xu, W., Roos, A., Schlagwein, N., Woltman, A.M., Daha, M.R., and van Kooten, C. (2006). IL-10-producing macrophages preferentially clear early apoptotic cells. Blood 107, 49304937.

Zhao, H., Kalish, F., Schulz, S., Yang, Y., Wong, R.J., and Stevenson, D.K. (2015). Unique Roles of Infiltrating Myeloid Cells in the Murine Uterus during Early to Midpregnancy. J Immunol 194, 3713-3722.

Zhao, J.X., Zeng, Y.Y., and Liu, Y. (2007). Fetal alloantigen is responsible for the expansion of the $\mathrm{CD} 4(+) \mathrm{CD} 25(+)$ regulatory $\mathrm{T}$ cell pool during pregnancy. J Reprod Immunol 75, 7181.

Zheng, W., and Flavell, R.A. (1997). The transcription factor GATA-3 is necessary and sufficient for Th2 cytokine gene expression in CD4 T cells. Cell 89, 587-596.

Zheng, X.F., Hong, Y.X., Feng, G.J., Zhang, G.F., Rogers, H., Lewis, M.A., Williams, D.W., Xia, Z.F., Song, B., and Wei, X.Q. (2013). Lipopolysaccharide-induced M2 to M1 macrophage transformation for IL-12p70 production is blocked by Candida albicans mediated up-regulation of EBI3 expression. PLoS One 8, e63967.

Zhu, J., and Paul, W.E. (2008). CD4 T cells: fates, functions, and faults. Blood 112, 1557 1569 .

Zhu, J., and Paul, W.E. (2010). Peripheral CD4+ T-cell differentiation regulated by networks of cytokines and transcription factors. Immunol Rev 238, 247-262.

Ziegler-Heitbrock, L., Ancuta, P., Crowe, S., Dalod, M., Grau, V., Hart, D.N., Leenen, P.J., Liu, Y.J., MacPherson, G., Randolph, G.J., Scherberich, J., Schmitz, J., Shortman, K., Sozzani, S., Strobl, H., Zembala, M., Austyn, J.M., and Lutz, M.B. (2010). Nomenclature of monocytes and dendritic cells in blood. Blood 116, e74-e80. 


\section{Papers}

The articles associated with this thesis have been removed for copyright reasons. For more details about these see:

http://urn.kb.se/resolve?urn=urn:nbn:se:liu:diva-117183 